\title{
RESIZING H.264/AVC FRAMES IN THE COMPRESSED DOMAIN
}

\author{
by
}

\author{
James McAvoy
}

\begin{abstract}
A thesis submitted to
the Faculty of Graduate Studies and Postdoctoral Affairs in partial fulfillment of the requirements for the degree of

Master of Computer Science

at

School of Computer Science

Carleton University

Ottawa, Ontario, Canada

May 2013
\end{abstract}

(C)Copyright 2013 James McAvoy 
Library and Archives

Canada

Published Heritage

Branch

395 Wellington Street

Ottawa ON K1A ON4

Canada
Bibliothèque et

Archives Canada

Direction du

Patrimoine de l'édition

395 , rue Wellington

Ottawa ON K1A ON4

Canada
Your file Votre référence

ISBN: $978-0-494-94614-5$

Our file Notre référence

ISBN: 978-0-494-94614-5
NOTICE:

The author has granted a nonexclusive license allowing Library and Archives Canada to reproduce, publish, archive, preserve, conserve, communicate to the public by telecommunication or on the Internet, loan, distrbute and sell theses worldwide, for commercial or noncommercial purposes, in microform, paper, electronic and/or any other formats.

The author retains copyright ownership and moral rights in this thesis. Neither the thesis nor substantial extracts from it may be printed or otherwise reproduced without the author's permission.
AVIS:

L'auteur a accordé une licence non exclusive permettant à la Bibliothèque et Archives Canada de reproduire, publier, archiver, sauvegarder, conserver, transmettre au public par télécommunication ou par l'Internet, prêter, distribuer et vendre des thèses partout dans le monde, à des fins commerciales ou autres, sur support microforme, papier, électronique et/ou autres formats.

L'auteur conserve la propriété du droit d'auteur et des droits moraux qui protege cette thèse. $\mathrm{Ni}$ la thèse ni des extraits substantiels de celle-ci ne doivent être imprimés ou autrement reproduits sans son autorisation.
In compliance with the Canadian Privacy Act some supporting forms may have been removed from this thesis.

While these forms may be included in the document page count, their removal does not represent any loss of content from the thesis.
Conformément à la loi canadienne sur la protection de la vie privée, quelques formulaires secondaires ont été enlevés de cette thèse.

Bien que ces formulaires aient inclus dans la pagination, il n'y aura aucun contenu manquant. 


\title{
CARLETON UNIVERSITY
}

DATE: May 8, 2013

\author{
AUTHOR: James McAvoy \\ TITLE: RESIZING H.264/AVC FRAMES IN THE COMPRESSED \\ DOMAIN
}

DEPARTMENT OR SCHOOL: School of Computer Science

DEGREE: M.C.S.

CONVOCATION: June

YEAR: 2013

Permission is herewith granted to Carleton University to circulate and to have copied for non-commercial purposes, at its discretion, the above title upon the request of individuals or institutions. I understand that my thesis will be electronically available to the public.

The author reserves other publication rights, and neither the thesis nor extensive extracts from it may be printed or otherwise reproduced without the author's written permission.

The author attests that permission has been obtained for the use of any copyrighted material appearing in the thesis (other than brief excerpts requiring only proper acknowledgement in scholarly writing), and that all such use is clearly acknowledged.

Signature of Author 


\section{Table of Contents}

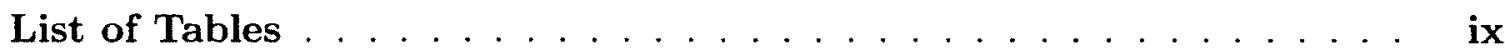

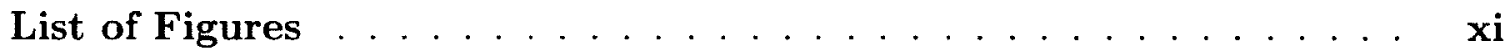

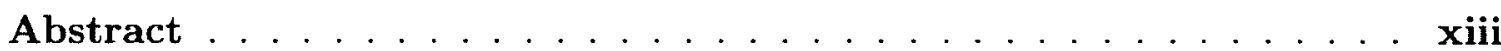

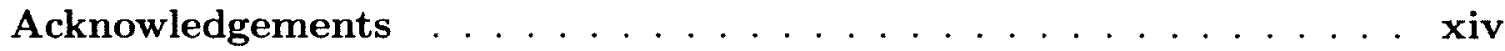

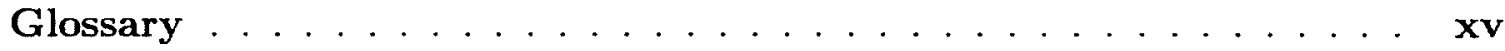

Chapter 1 Introduction $\ldots \ldots \ldots \ldots \ldots \ldots$

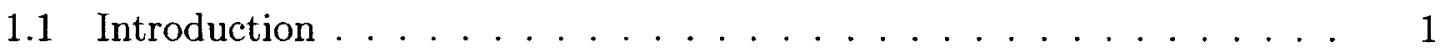

1.2 Resizing Video Frames or Images in the DCT Domain . . . . . . . . 1

1.3 Motivation and Problem Description $\ldots \ldots \ldots \ldots$

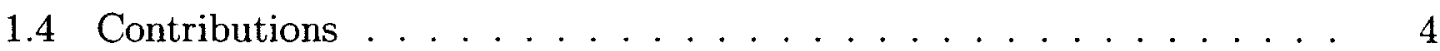

1.5 Thesis Scope . . . . . . . . . . . . . . . . . . . . 6

1.6 Organization of the Thesis $\ldots \ldots \ldots \ldots \ldots \ldots$

Chapter 2 Background $\ldots \ldots \ldots \ldots \ldots \ldots$

2.1 Introduction . . . . . . . . . . . . . . 7

$2.2 \quad$ H.264/AVC Standard . . . . . . . . . . . . . . . 7

2.2 .1 H.264 Transform . . . . . . . . . . . . . . . 9 9

2.2 .2 Quantization ... . . . . . . . . . . . . . 11

2.2.3 Developing the Forward Transform and Quantization Process . 12

2.2.4 Developing the Inverse Transform Process . . . . . . . . . 14

2.3 Performance Measurements . . . . . . . . . . . . . . . 14

2.3.1 Peak Signal-to-Noise Ratio (PSNR) . . . . . . . . . . 15

2.3.2 Computational Cost . . . . . . . . . . . . . . . 16

2.4 Resizing an Image in the Spatial Domain . . . . . . . . . . . 17

2.4.1 Computational Cost when Halving an H.264/AVC Frame in the Spatial Domain . . . . . . . . . . . . . . 18

2.5 Subband DCT Computation . . . . . . . . . . . . . . . 20

2.5.1 Approximate DCT Computation . . . . . . . . . . . 21 
2.5.2 Approximate DCT in 2-D .............. 21

2.6 Composition and Decomposition of Blocks of DCT Coefficients . . . . 22

2.7 Summary . . . . . . . . . . . . . . . . . . . 24

Chapter 3 DCT Domain Image Resizing Algorithms . . . . . . . 25

3.1 Introduction . . . . . . . . . . . . . . 25

3.2 Image Halving . . . . . . . . . . . . . . . . . 26

3.2.1 Image Halving through Bilinear Interpolation (IHBI) . . . . . 26

3.2.2 Image Halving through Low-Pass Filtering followed by Downsampling (IHFDS) . . . . . . . . . . . 27

3.2.3 Image Halving through Approximation followed by Composition (IHAC) . . . . . . . . . . . . . 30

3.2.4 Image Halving through Composition followed by Approximation $(\mathrm{IHCA}) \ldots \ldots \ldots . \ldots . \ldots . \ldots 31$

3.3 Image Doubling . . . . . . . . . . . . . . . . . . 32

3.3.1 Image Doubling through Zero-Order Interpolation (IDZOI) . . 32

3.3.2 Image Doubling through Upsampling followed by Zero Padding (IDUSZP) . . . . . . . . . . . . . . . . 33

3.3.3 Image Doubling through Decomposition followed by Approximation (IDDA) . . . . . . . . . . . . 34

3.3.4 Image Doubling through Approximation followed by Decompo-

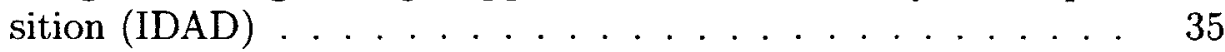

3.4 Resizing with Integral Factors . . . . . . . . . . . . . . 36

3.4.1 $L \times M$ Downsampling Algorithm (LMDS) . . . . . . . . . 37

3.4.2 $L \times M$ Upsampling Algorithm (LMUS) . . . . . . . . . . 37

3.5 Resizing with Arbitrary Rational Factors . . . . . . . . . . . . . . . 38

3.5.1 UD-Resizing and DU-Resizing Algorithm . . . . . . . . 39

3.6 Hybrid Resizing Algorithm . . . . . . . . . . . . . . . . 39

3.7 Summary . . . . . . . . . . . . . . . . . . 41

Chapter 4 DCT Domain H.264/AVC Frame Resizing Algorithms 42

4.1 Introduction . . . . . . . . . . . . . . . . . 42

4.2 Halving H.264/AVC Frames . . . . . . . . . . . . . . . 42

4.2.1 Image Halving through Bilinear Interpolation (IHBI) . . . . . 42

4.2.2 Image Halving through Low-Pass Filtering followed by Downsampling (IHFDS) . . . . . . . . . . . 43 
4.2.3 Image Halving through Approximation followed by Composi-

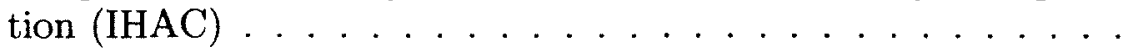

4.2.4 Image Halving through Composition followed by Approximation $(\mathrm{IHCA}) \ldots \ldots \ldots \ldots \ldots . \ldots \ldots$

4.3 Doubling H.264/AVC Frames . . . . . . . . . . . . . . 47

4.3.1 Image Doubling through Zero-Order Interpolation (IDZOI) . 48

4.3.2 Image Doubling through Upsampling followed by Zero-Padding (IDUSZP) . . . . . . . . . . . . . . . . . . 49

4.3.3 Image Doubling through Decomposition followed by Approximation (IDDA) . . . . . . . . . . . . . .

4.3.4 Image Doubling through Approximation followed by Decomposition $(\mathrm{IDAD}) \ldots \ldots \ldots \ldots \ldots \ldots$

4.4 Resizing with Integral Factors $\ldots \ldots \ldots \ldots \ldots$

4.4.1 $L \times M$ Downsampling Algorithm (LMDS) $\ldots \ldots \ldots \ldots \ldots 53$

$4.4 .2 L \times M$ Upsampling Algorithm (LMUS) . . . . . . . . . 54

4.5 Resizing with Arbitrary Factors $\ldots \ldots \ldots \ldots \ldots$

4.6 Hybrid Approach . . . . . . . . . . . . . . . 56

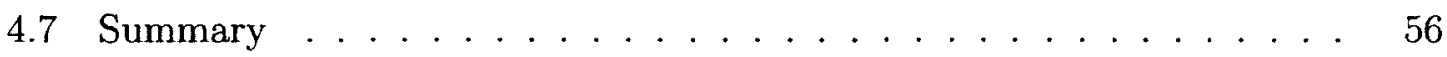

Chapter 5 General Description of Experimental Environment . . 57

5.1 Introduction . . . . . . . . . . . . . . . . 57

5.2 The Image Sample Set and H.264/AVC File Representation _ . . . 57

5.3 The DCT Domain H.264/AVC Frame Resize Algorithms . . . . . . 57

$5.3 .1 \mathrm{C}++$ Linear Algebra Library $\ldots \ldots \ldots \ldots$

5.4 PSNR Measurements . . . . . . . . . . . . . . . . . . . 59

5.5 Computational Cost $\ldots \ldots \ldots \ldots \ldots \ldots$

5.6 Raw Image Viewer $\ldots \ldots \ldots \ldots \ldots$

5.7 Automation . . . . . . . . . . . . . . . . 60

5.8 MATLAB for Resizing Images in the Spatial Domain . . . . . . . 61

5.9 Summary $\ldots \ldots \ldots \ldots \ldots \ldots$

Chapter 6 Experimental Results from Resizing H.264/AVC Frames in the DCT Domain . . . . . . . . . . . . . . . 62 


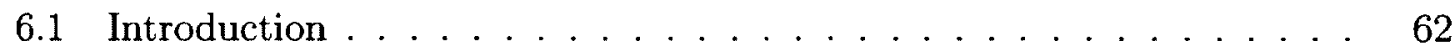

6.2 Image Quality . . . . . . . . . . . . . . . . . . . . 62

6.2.1 Image Halving and Doubling Algorithms Performance . . . . . 62

6.2 .2 Integral Resizing Algorithms . . . . . . . . . . . . . . 67

6.2 .3 Arbitrary Resizing Algorithms . . . . . . . . . . . . 69

6.3 Computational Cost . . . . . . . . . . . . . . . . . . . 71

6.3.1 Computational Cost for Image Halving and Doubling Resize

Algorithms . . . . . . . . . . . . . . . 73

6.3.2 Computational Cost for Resizing Algorithms with Integral Factors 74

6.3.3 Computational Cost for Arbitrary Resizing Algorithms . . . 75

6.4 Summary of Results . . . . . . . . . . . . . . . . 76

Chapter 7 Removing Quantization Step in the DCT Domain Resizing Process . . . . . . . . . . . . . 78

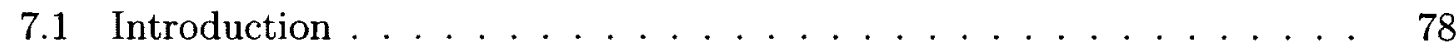

7.2 Creating Quantization Matrix . . . . . . . . . . . . 79

7.2 .1 Example . . . . . . . . . . . . . . 80

7.3 Properties of Conversion Matrix . . . . . . . . . . . . . . 82

7.4 Experimental Setup . . . . . . . . . . . . . . . . . . 84

7.5 Presentation of Experimental Results . . . . . . . . . . . . 85

7.6 Analysis of Results . . . . . . . . . . . . . . . 86

Chapter 8 Efficient Resizing Algorithms in the DCT Domain . . 88

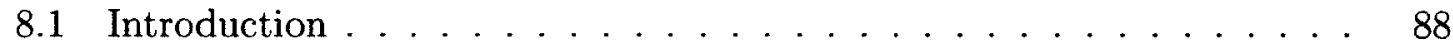

8.2 Experimental Results . . . . . . . . . . . . . . . . . . 92

8.3 Computational Cost $\ldots \ldots \ldots \ldots \ldots \ldots \ldots$

8.4 Analysis of Results $\ldots \ldots \ldots \ldots \ldots$

Chapter 9 Conclusions and Future Work $\ldots \ldots \ldots$

9.1 Introduction . . . . . . . . . . . . . . . . 99

9.2 Concluding Remarks $\ldots \ldots \ldots \ldots \ldots$

9.3 Future Work . . . . . . . . . . . . . . . . . . . . 100 
Appendix A PSNR for Image Halving and Doubling Experiments . 102

Appendix B PSNR for Image Resizing by Integral Factors . . . . 105

Appendix C PSNR for Arbitrary Resizing Algorithms . . . . . . 107

Appendix D Image Sample Set . . . . . . . . . . . . . . 109

Appendix E Hybrid Resizing Algorithm (HRA) Source Code . . . 110

Bibliography ...................... 111 


\section{List of Tables}

6.1 Experiment 1. A summary of the mean PSNR values for each

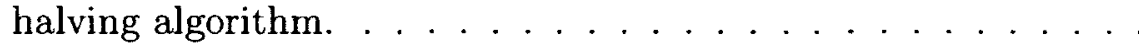

6.2 Experiment 2. A summary of the mean PSNR values for each doubling algorithm and difference in $\mathrm{dB} \ldots . . . . . .$.

6.3 Experiment 3. A summary of the mean PSNR values after image halving followed by image doubling and difference in $\mathrm{dB}$ from IHS-IDS resizing. . . . . . . . . . . . . . . . .

6.4 Computation cost of image halving algorithms per pixel of the original image . . . . . . . . . . . . . .

6.5 Computation cost of image doubling algorithms per pixel of the upsampled image . . . . . . . . . . . . . .

6.6 LMDS Computational cost per pixel of the original image . . .

6.7 LMUS computational cost per pixel of the upsampled image . .

6.8 Computational cost of arbitrary resizing algorithms per pixel of the original image and the quality/cost ratio . . . . . . . . .

7.1 The mean PSNR value with standard deviation $\sigma$ when comparing DCT domain resizing processes that includes quantization and one without quantization over multiple QP levels. . . . . .

7.2 The mean bitrate with standard deviation $\sigma$ when comparing DCT domain resizing processes that includes quantization and one without quantization over multiple QP levels. The last column outlines the bitrate saving if process includes quantization.

8.1 Results from three experiments used to evaluate image quality performance of IIHAC and IIDDA resize algorithms. . . . . .

IIHAC Account of Computations . . . . . . . . . . .

8.3 IIDDA Account of Computations . . . . . . . . .

8.4 Comparing computation cost Integer based IHAC (IIHAC) with other image halving algorithms by the number of multiplication $\left(n_{m}\right)$ and additions $\left(n_{a}\right)$ per pixel of the original image .... 
8.5 Comparing computation cost Integer based IDDA (IIDDA) with other image doubling algorithms by the number of multiplication $\left(n_{m}\right)$ and additions $\left(n_{a}\right)$ per pixel of the upsampled image ...

A.1 Experiment 1. PSNR values computed by comparing an image halved in the spatial domain using bicubic interpolation with anti-aliasing against images generated from various halving algorithms. . . . . . . . . . . . . . .

A.2 Experiment 2. PSNR values computed by comparing the original images with images that were doubled from compressed halved images using various doubling algorithms. Bicubic interpolation with anti-aliasing was used to halve the original images to create the compressed halved images. . . . . . . . . . . . .

A.3 Experiment 3. PSNR values computed by comparing the original images with images generated from various halving and doubling algorithms. . . . . . . . . . . . . . . 104

B.1 Experiment 4. PSNR values for LMDS and LMUS algorithms. 105

C.1 Experiment 5. PSNR values after arbitrary resizing using various scaling factors and algorithms. . . . . . . . . . 107

C.2 Experiment 6. PSNR values after arbitrary resizing followed by inverse resizing. . . . . . . . . . . . . . . 108

D.1 Images that are members of the sample set. . . . . . . . 109 


\section{List of Figures}

1.1 The straight forward approach of resizing an image or video frame, where $x$ is the original image and $\hat{x}$ is the resized image. 2

1.2 The processing flow of resizing images or video frame in the DCT domain, where $x$ is the original image and $\hat{x}$ is the resized image. . . . . . . . . . . . . . .

1.3 A resizing H.264/Frame without quantization step, where $x$ is the original frame and $\hat{x}$ is the resized frame. . . . . . . 5

2.1 A high level overview of a H.264/AVC encoder and decoder [15]. 10figure.2.2

2.3 Downsampling four adjacent $8 \times 8$ DCT blocks by a factor of two to get a single $8 \times 8$ DCT block in the spatial domain. . .

3.1 Schematic of the low-pass filtering followed by downsampling approach developed by Dugad and Ahuja [12] . . . . . . . 28

3.2 Image Doubling through Zero-Order Interpolation. . . . . . .

4.1 Image doubling through zero-order interpolation. . . . . . .

5.1 RawViewer application displaying the original Lena image on the left and Lena image halved on the right. . . . . . . . . .

6.1 Figure 6.2(a) is an image half in the spatial domain using bilinear interpolation with PSRN value of 42.6. Figure 6.1(b) IHBI, Figure 6.1(c) IHAC, Figure 6.1(d) IHFDS and Figure 6.1(e) IHCA. The PSNR values are $38.79,40.51,40.57$, and 36.53 respectively. . . . . . . . . . . . . .

6.2 Watch image: 6.2(a) Original. Portion of the resultant images from experiment 3 , where images were first halved then doubled: 6.2(b) Spatial resizing, 6.2(c) IHAC-IDDA, 6.2(d) IHFDSIDUSZP, 6.2(e) IHCA-IDAD, 6.2(f) IHBI-IDZOI. The PSNR values with respect to the original are $25.98,27.69,27.67,29.14$ and $25.51 \mathrm{~dB}$ respectively.

6.3 A chart showing the mean PSNR value for each image when scaled by various factors $L \times M$ based on experiment 4 . . . . 
6.4 Images generated from applying LMDS algorithm. Figure 6.4(a) displays the original image. Figure $6.4(\mathrm{~b})$ is an image scaled by a factor of $2 \times 3$. Figure $6.4(\mathrm{c})$ is scaled by $3 \times 2$ and Figure $6.4(\mathrm{~d})$ by $3 \times 4$. The computed PSNR values when compared with an image resized in the spatial domain is $30.71,30.36$ and $30.58 \mathrm{~dB}$ respectively. . . . . . . . . . . . . .

6.5 A summary of the mean PSNR values $(\mathrm{dB})$ that was computed over a sample set obtained in experiment 6 , where the image was first resized by arbitrary factor followed by inverse resize operation to return the image to its original resolution. . . .

6.6 Sample images produced from experiment 6 , where images were arbitrary resizing by a factor $\frac{3}{2} \times \frac{5}{4}$ followed by inverse resizing. Figure 6.6(a) is the original image. Notice DURA created image displayed in Figure 6.6(c) suffers from blocking artifacts. . .

7.1 The processing flow of resizing images or video frame in the DCT domain, where $x$ is the original image and $\hat{x}$ is the resized image. . . . . . . . . . . . . . . .

7.2 A resizing H.264/Frame without quantization step, where $x$ is the original frame and $\hat{x}$ is the resized frame. . . . . . . .

7.3 The comparing default forward h.264/AVC transform process with regular DCT transforms with quantization. . . . . . . .

8.1 IHAC [13]. Four $\frac{N}{2} \times \frac{N}{2}$ approximated DCT coefficients of adjacent blocks are composed into one $N \times N$ DCT block. . . .

8.2 IDDA [13]. An $N \times N$ DCT block is decomposed into four $\frac{N}{2} \times \frac{N}{2}$ blocks where each is approximated to an $N \times N$ DCT block with zero-padding. . . . . . . . . . . . .

8.3 Figure 8.3(a) is an image halved in the spatial domain using IHS algorithm where the following resultant images from halving algorithms are compared with: Figure 8.3(b) integer based IIHAC and Figure 8.3(c) floating-point based IHAC. The PSNR values are 40.63 and 43.01 respectively.

8.4 Average image quality versus number of multiplications of various resizing algorithms. . . . . . . . . . . . . 


\begin{abstract}
One of the most popular standards to encode video is H.264/Advanced Video Coding(AVC), which provides a compression gain of $1.5 \times$ to $2.0 \times$ over previous standards such as MPEG2 or H.263+. Resizing H.264/AVC video for storage or transmission is often required because of imposed constraints dictated by network bandwidth or device capabilities. Usually, systems resize H.264/AVC video in the spatial domain. However, it would be more attractive to resize the video directly in the compressed format, which would avoid high computational overhead associated with decompression and compression operations. This thesis proposed methods to resize H.264/AVC frames that leveraged contributions made in image and video resizing in the DCT domain. We also proposed a new efficient DCT resizing algorithm that exploits fixedpoint integer transforms. This approach has shown to reduce the computational cost by more than $60 \%$ with small $\mathrm{dB}$ loss when comparing similar algorithms using floating-point transforms.
\end{abstract}




\section{Acknowledgements}

I first like to thank my thesis adviser, Professor Chris Joslin, for his guidance, support and patience in showing me an area of research that inspired me to write this thesis. I also would like to thank Francois Urbain, my work supervisor, for supporting me on my effort in pursuing my Master's degree. Finally and most important of all, I am grateful to my wife, Sherry, who provide constant support, patience and encouragement during my journey. 


\section{Glossary}

CAVLC Context Adaptive Variable Length Coding

DCT Discrete Cosine Transform

DST Discrete Sine Transform

DURA DU-Resizing Algorithm

HRA Hybrid Resizing Algorithm

IDAD Image Doubling through Approximation followed by Decomposition

IDBI Image Doubling through Bilinear Interpolation

IDDA Image Doubling through Decomposition followed by Approximation

IDFDS Image Halving through low-pass Filtering followed by Downsampling

IDUSZP Image Doubling through Upsampling followed by Zero-Padding

IHAC Image Halving through Approximation followed by Composition

IHBI Image Halving through Bilinear Interpolation

IHCA Image Halving through Composition followed by Approximation

LMDS $\quad L \times M$ Downsampling Algorithm

LMUS $L \times M$ Upsampling Algorithm

PSNR Peak Signal-to-Noise Ratio 
QP Quantization Parameter

UDRA UD-Resizing Algorithm 


\section{Chapter 1}

\section{Introduction}

\subsection{Introduction}

Since ITU/ISO first published the H.264 Advanced Video Coding (AVC)[1][2] recommendation in 2003, it has emerged as a dominant compression standard in several application domains. Especially, in the mobile phone arena where video content are transmitted over disadvantaged data networks and are consumed by devices with limited capacity. The preference of employing H.264/AVC over previous standards to encode video is not surprising. Applications using H.264/AVC over older standards will benefit from a $50 \%$ bit rate saving or greater for the same picture quality [3]. In a study on digital TV quality over satellites, researchers [4] encoded H.264/AVC video at a bitrate of $1.5 \mathrm{Megabits} / \mathrm{second}(\mathrm{Mbit} / \mathrm{s}$ ) for the same picture quality as an MPEG 2 video that required $3.5 \mathrm{Mbit} / \mathrm{s}$. Even though H.264/AVC has greater encoding efficiency than previous standards, the video may be still too large to be transmitted over bandwidth disadvantaged networks.

\subsection{Resizing Video Frames or Images in the DCT Domain}

Systems delivering video over disadvantaged data networks can be a challenge. When operating in that type of network environment, applications need to adapt the precoded or real-time video to meet a broad range of end-user with different bandwidths and device capabilities. Streaming full resolution 1080p HD video over wireless mobile networks to hand-held devices will exceed the network's bandwidth and the device's resources such as screen size, $\mathrm{CPU}$ and memory.

One strategy to transmit video over bandwidth constrained networks is to reduce the video's spatial resolution and transmit the low-resolution version of the video as trade off to bitrate. The study of image and video frame resizing deals with the conversion of an image of size $M_{1} \times N_{1}$ to a different size of $M_{2} \times N_{2}$. If both $M_{2}$ and 


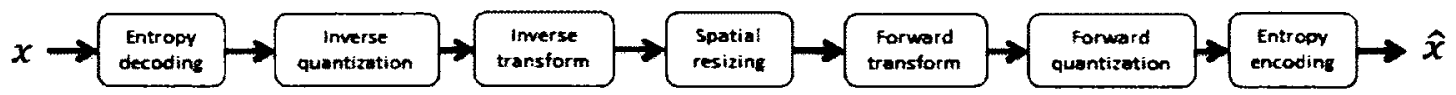

Figure 1.1: The straight forward approach of resizing an image or video frame, where $x$ is the original image and $\hat{x}$ is the resized image.

$N_{2}$ are less than $M_{1}$ and $N_{1}$, the process is called downsampling. On the other hand, if both $M_{2}$ and $N_{2}$ are greater than $M_{1}$ and $N_{1}$, then the process is called upsampling. In this thesis, we constrained our research on video frame or image resizing. To fully transcode video sequence from one spatial resolution to a desired spatial resolution requires us to transcode other coding elements associated with video compression, such as motion compensation.

An obvious approach to changing a video's resolution would be to resize the frames in the spatial domain. As illustrated in Figure 1.1, this straightforward approach of: 1) entropy decoding, 2) inverse quantization, 3) inverse transform, 4) spatial domain resizing, 5) forward transform, 6) quantization and 7) entropy encoding to the required resolution is undesirable due to inherent high computational cost. Also, spatial resizing of compressed frames degrades the picture quality because of decompression and compression process.

To improve the resizing process, early researchers developed algorithms to work directly on the compressed image ${ }^{1}$ in the Discrete Cosine Transform (DCT) domain. This approach proved to decrease computational cost by reducing the number of steps in the resizing process. As shown in Figure 1.2, several papers [5][6][7][8][9][10] in this research area would described a typical processing flow of image or video frame resizing from one spatial resolution to a different spatial resolution to contain the following steps: 1) entropy decoding, 2) inverse quantization, 3) DCT domain resizing, 4) forward quantization and 5) entropy encoding. When comparing this approach with the straight forward approach presented in Figure 1.1, the inverse and forward transform steps are removed, which contributes most of the computational cost in image or video frame resizing.

\footnotetext{
${ }^{1}$ Image and video frames will be used interchangeably in this thesis.
} 


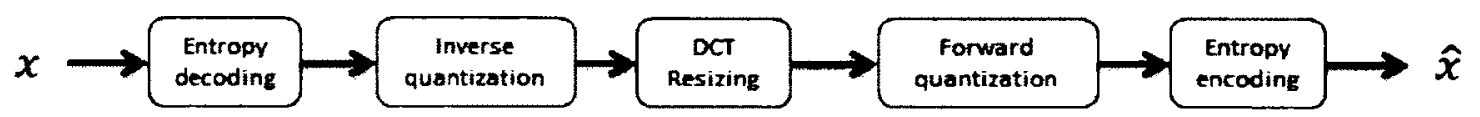

Figure 1.2: The processing flow of resizing images or video frame in the DCT domain, where $x$ is the original image and $\hat{x}$ is the resized image.

The basic framework of most of these algorithms is to create a mapping that transforms blocks of DCT coefficients to the desired spatial resolution that are encoded as blocks of DCT coefficients. This mapping is usually performed using matrices that are multiplied with the blocks of DCT coefficients where the product is blocks of DCT coefficients. In most cases, the algorithms will apply some pre or post operations to the blocks by either decimating the DCT coefficients or adding zero padding to the DCT block. In other cases, these pre or post operations are incorporated into the mapping. Chapter 3 will provide a more detailed explanation on how these algorithms work.

To determine if these DCT resize algorithms had any practical value, most researchers would apply these algorithms on JPEG images or MPEG2 frames, which were dominant compression standards before H.264/AVC. These early standards applied a $8 \times 8$ DCT to transform spatial domain pixels into the frequency domain to form blocks of $8 \times 8$ DCT coefficients. Therefore, most researchers would describe their algorithms based on resizing images encoded in $8 \times 8$ DCT block framework. Papers on DCT resizing presented computational efficient algorithms generating images that are of equal or better quality than comparable spatial domain techniques. Our research goal is to leverage these contributions and adapt these algorithms to resize H.264/AVC frames.

\subsection{Motivation and Problem Description}

As previously stated, most research in the area of resizing images and video in the DCT domain assumed multimedia products are compressed using a popular $8 \times 8$ 
block DCT framework. However, H.264/AVC deviates from previous video compression standards. When transforming an image from the spatial to the frequency domain, H.264/AVC employs $4 \times 4$ integer transform, an approximate form of the DCT, to form blocks of $4 \times 4$ DCT coefficients. Also in earlier standards, the boundary between transform and quantization operations were obvious but in H.264/AVC these boundaries are unclear with overlapping transform and quantization stages [1][2].

Our research goal was to develop methods to resize H.264/AVC video frames based on DCT image resize algorithms that were originally developed for $8 \times 8$ DCT framework. The benefit will be faster video resizing with image quality similar or better than comparable operations carried out in the spatial domain.

\subsection{Contributions}

To meet our objective of developing methods to resize H.264/AVC video frames in the compressed domain, the thesis proposed the following contributions:

a) Designing algorithms to resize H.264/AVC frames in the DCT space. We developed algorithms to resize H.264/AVC video frames in the DCT domain that are based on $8 \times 8$ DCT resize algorithms. The following thirteen DCT resizing algorithms have been studied and adapted to resize H.264/AVC frames:

IHBI [7][11] Image Halving through Bilinear Interpolation.

IDZOI [7][11] Image Doubling through Zero-Order Interpolation.

IHFDS [12] Image Halving through Low-Pass Filtering followed by Downsampling.

IDUSZP [12] Image Doubling through Upsampling followed by Zero-Padding.

IHAC [13] Image Halving through Approximation followed by Composition.

IDDA [13] Image Doubling through Decomposition followed by Approximation.

IHCA [13] Image Halving through Composition followed by Approximation.

IDAD [13] Image Doubling through Approximation followed by Decomposition. 
LMDS [13] $L \times M$ Downsampling Algorithm.

LMUS [13] $L \times M$ Upsampling Algorithm.

UDRA [13][14] UD-Resizing Algorithm.

DURA [13][14] DU-Resizing Algorithm.

HRA [14] Hybrid Resizing Algorithm.

Most of these algorithms generated images that are similar and greater in quality at lower computational cost than comparable spatial domain techniques.

b) Quantization step can be removed from the resizing process. The default forward and inverse H.264/AVC transform incorporates quantization and scaling. Because H.264/AVC blends transform and quantization, can we skip the inverse and forward quantization steps? If so, a revised processing flow for resizing can be used that contains the following steps: 1) entropy decoding, 2) DCT domain resizing, and 3) entropy encoding, which is shown in Figure 1.3. It will be demonstrated that this new resizing process can reduce computational cost and without sacrificing image quality. Also, the bitrate saving for a process that includes quantization is negligible.

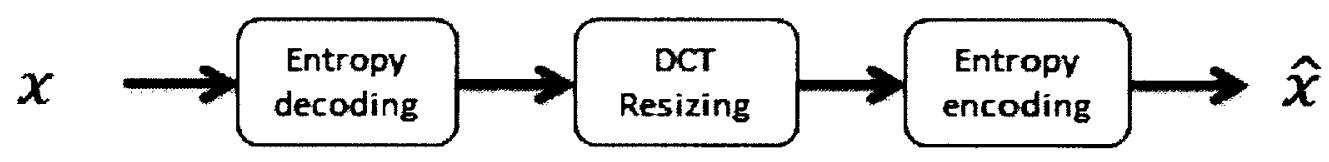

Figure 1.3: A resizing H.264/Frame without quantization step, where $x$ is the original frame and $\hat{x}$ is the resized frame.

c) Efficient resizing algorithms in the DCT domain. We discovered that a certain class of DCT resize algorithms employ orthonormal matrices to transform coefficients from one vector space to an other vector space of the desired resolution. Most of these transform matrices are sparse and all of them contain entries that require processors to approximate irrational numbers, which adds to the computational cost. We developed an integer approximation of these transforms that reduced the computational cost by over $60 \%$ when comparing to its 
floating-point version. From experiments, we observed slight image quality loss of approximately $0.3 \mathrm{~dB}$ loss over our sample set.

\subsection{Thesis Scope}

One can view video compression as image compression with a temporal component since video consists of a sequence of images over time. To achieve greater compression, a number of coding elements working in concert is required, such as prediction and motion compensation. For this thesis, we restricted ourselves with the problem of image or frame resizing. In other words, the problem of resizing compressed I Frames.

Most image and video compression standards are based on a DCT block framework. Therefore, we will often used the terms image and video frames interchangeably, because algorithms developed to resize images are applicable to resizing video frames.

\subsection{Organization of the Thesis}

To understand resizing H.264/AVC frames in the compressed domain, a short introduction to the H.264/AVC standard is presented in Chapter 2. Only the coding elements that are related to frame encoding are covered. The background section outlines performance measures for computational cost. Several researchers employ two computational techniques in several resizing algorithms: 1) subband DCT computation, and 2) composition and decomposition of DCT blocks. Chapter 2 will present a short overview of these methods.

Several DCT resizing algorithms will be covered in Chapter 3. Most of these resizing algorithms will be adapted to work on H.264/AVC frames and will be presented in Chapter 4. A general description of experimental procedure will be presented in Chapter 5. In Chapter 6, experimental results from resizing H.264/AVC frames using different DCT algorithms are presented. Chapter 7 describes how quantization step can be removed from the resizing process. Chapter 8 outlines computational efficient DCT resize algorithm that we developed. Conclusions and future areas of study are provided in the last chapter. 


\section{Chapter 2}

\section{Background}

\subsection{Introduction}

This chapter covers topics that are required to understand how to resize H.264/AVC frames in the compressed domain. We will first introduce the H.264/AVC standard but only covering the coding elements that are related to resizing frames in the compressed domain. Measuring the performance of these resizing algorithms is important so sections on calculating computational cost and image quantity are covered. Then we will provide a short example on resizing an image in the spatial domain to show the computational cost. Most of the algorithms in this thesis combine two techniques to resize compressed images: 1) subband DCT computation, and 2) composition and decomposition of DCT blocks. The first method helps us extract the lower frequency coefficients from an $N \times N$ DCT block of coefficients. The second method shows the relationship between $N \times N$ block of coefficients with its spatial representation. The ability to change the block coefficients to a new basis vector, which can be of higher or lower dimensions, is covered in the section on composition and decomposition of DCT blocks.

\subsection{H.264/AVC Standard}

H.264/AVC is an industrial standard for lossy video compression. Being a lossy compression standard, some information is discarded from the original source data for the sake of compression. H.264/AVC is a joint ITU/ISO standard that describes a method and format for video compression. As shown in Figure 2.1, the standard only covers the format and syntax of a valid H.264/AVC bitstream and the decoding process. The standard document does not inform encoder manufactures how to code a valid H.264/AVC bitstream. In practice, most encoder manufactures mirror the steps in the decoding process. 


\section{VIDEO ENCODER}

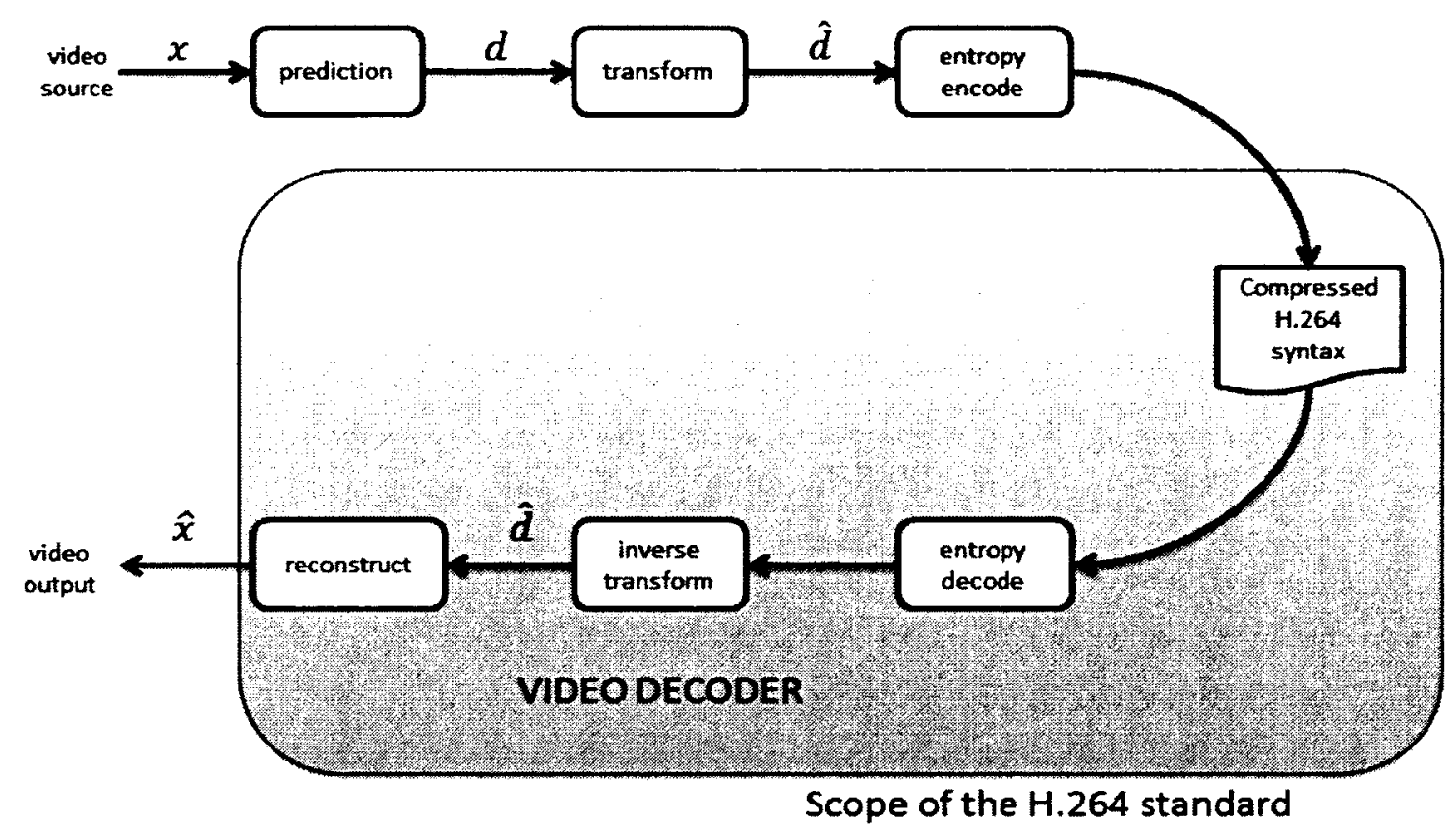

Figure 2.1: A high level overview of a H.264/AVC encoder and decoder [15].

Video compression can be viewed as image compression with temporal component. In the encoder block diagram displayed in Figure 2.1, the input source signal $x$ represents one image in a video sequence. Each image contains a sequence of pixels $x[n]$ arranged over two dimensional planes.

After the prediction process, a residual image $d$ is created, which is transformed and quantized to create coefficients $\hat{d}$. The encoder entropy encodes the coefficients $\hat{d}$ used to create a valid H.264/AVC bitstream.

An H.264/AVC decoder carries out the complementary steps of decoding, inverse transform and reconstruction to produce a decoded video sequence $\hat{x}$ to be displayed onto a monitor.

In Figure 2.1, the coding element transform contains more operations. In previous standards, the encoder would apply a quantization process after the forward transform and for the case of the decoder a de-quantization process is applied before the inverse transform. However, these boundaries of transform and quantization are not 
obvious in H.264 but overlap. The coefficients mapping from the spatial domain to the frequency domain are first transform using integer DCT then quantization process followed by scaling. In past research on DCT domain image resizing, researchers assumed that the blocks of DCT coefficients are de-quantized.

Entropy coding and prediction process are not required background information to understand resizing images or video frames in the DCT domain. They are important coding elements but general understanding where these processes are located in the coding sequence is all that is necessary to understand resizing in the compressed domain. In the next subsections, H.264/AVC transform and quantization will be covered.

\subsubsection{H.264 Transform}

In most images, pixels are highly correlated with their neighbouring pixels. To break this correlation, the image $x$ is transformed into sub-source coefficients $\theta$, see Figure 2.2. One of the central tenets of transform coding is that uncorrelated data can be compressed more efficiently than correlated data before it is quantized and entropy encoded [16][17]. This transform process is lossless, which means the process can be reverse without any loss of information. The compression really occurs during the quantization $\mathcal{E}$ and entropy encoding $\mathcal{C}$ process. After entropy encoding, the encoder multiplexes the subsources to create a compliant H.264/AVC bitstream so it can be serialized for storage or transmission. The H.264/AVC decoder reads the video bitstream and does the complementary process of de-multiplexing, decoding, de-quantizing and inverse transform to reconstruct a valid video sequence for display.

Other video or image compression standards, such as JPEG, MPEG2 and MPEG4, specify Discrete Cosine Transform [18] to be applied to the source and residual image data. The standards would define the inverse transform as shown in Equation (2.1) for a block size $N \times N$ where $Y_{x y}$ are the input coefficients and $X_{i j}$ are the output image or residual samples:

$$
X_{i j}=\sum_{x=0}^{N-1} \sum_{y=0}^{N-1} C_{x} C_{y} Y_{x y} \cos \frac{(2 j+1) y \pi}{2 N} \cos \frac{(2 i+1) x \pi}{2 N}
$$

The implementation of the above equation for $N>2$ on a common processor 

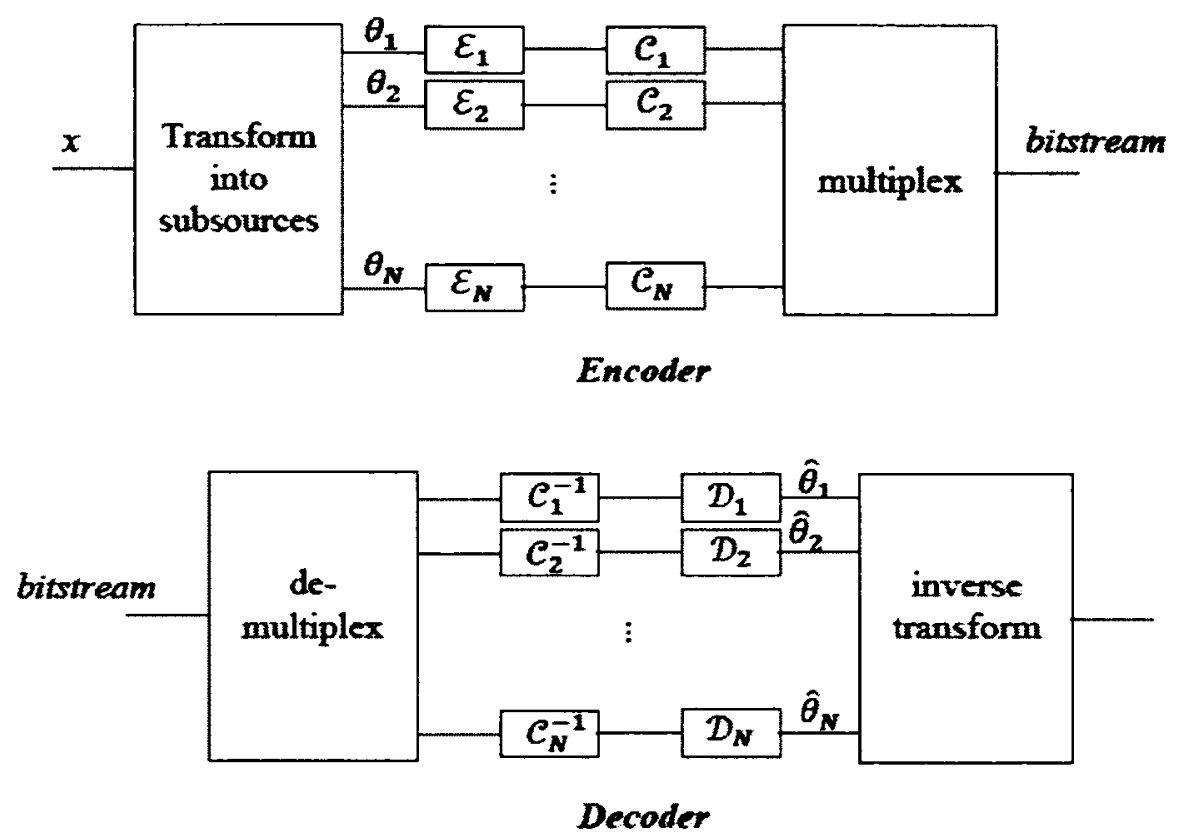

Figure 2.2: Transform coding overview ${ }^{a}$.

\footnotetext{
${ }^{a}$ In courtesy of Dr. Eric Dubois from course notes on Source Coding and Data Compression. Copyright, all rights reserved.
}

requires approximations to certain irrational multiplication factors, $\cos (a \pi / 2 N)$. Encoders and decoders would implement different approximations of the above equation, which can change the output of the forward and inverse transforms. Overtime, this would lead to 'drift' or cumulative distortion it the decoder output. To compensate for the mismatch, more intra coding refresh must be introduce into the video sequence; therefore, increasing the bitrate.

In H.264/AVC, the standard members intentionally designed the transform and quantization processes to be computationally simple and suited for implementation using limited-precision integer arithmetic. To achieve this, the standard exactly specifies the inverse transform process to use a DCT-like matrix shown in (2.2) to be carried 
out by a decoder.

$$
\mathbf{C}_{i 4}=\left[\begin{array}{cccc}
1 & 1 & 1 & \frac{1}{2} \\
1 & \frac{1}{2} & -1 & -1 \\
1 & -\frac{1}{2} & -1 & 1 \\
1 & -1 & 1 & -\frac{1}{2}
\end{array}\right]
$$

This ensures that every H.264 decoder implementation should produce identical results and eliminate mismatch between different transform implementations.

As stated earlier in the thesis, the standard does not specify how encoders are to be built, only the decoders. However, most encoders implement the following forward transform matrix shown in (2.3).

$$
\mathbf{C}_{f 4}=\left[\begin{array}{cccc}
1 & 1 & 1 & 1 \\
2 & 1 & -1 & -2 \\
1 & -1 & -1 & 1 \\
1 & -2 & 2 & -1
\end{array}\right]
$$

Using these matrices, transform process can be implemented using additions and shifts for multiplication and division operations. As you may notice the transform matrix size is $4 \times 4$, which is smaller than the image or residual macroblocks of $16 \times 16$. The smaller size provides better representation of small stationary regions in the image and contains smaller variance of values in a block. The standard provides an optional $8 \times 8$ transform block, but is only available for High profile decoders [2][1].

These matrices stated in (2.2)(2.3) are often referred as the core transforms, which are a scaled approximation of the very popular Discrete Cosine Transform, DCT. If the forward and inverse core transforms are multiplied, the result is not an identity matrix. In other words:

$$
\mathrm{C}_{f 4} \cdot \mathbf{C}_{i 4} \neq I
$$

This discrepancy will be corrected during the quantization process, which will be discussed in the next section.

\subsubsection{Quantization}

The basic quantization process is fairly straight forward. The output of the transform process is a block of transform coefficients $\theta$ as shown in Figure 2.2 on transform 
coding overview. The encoder quantized each coefficient which is the process of dividing the coefficient by an integer value. The quantization process reduces the precision of the transform coefficients according to a quantization parameter (QP). Setting a high QP will introduce greater distortion because most of coefficients will be set to zero, while lower QP will mean more non-zero coefficients will remain after the quantization. This results in better image quality but lower compression.

In earlier standards, the boundary between transform and quantization process were obvious. However, in H.264/AVC these boundaries are unclear with overlapping transform and quantization stages. As discussed in the previous section, H.264/AVC standard explicitly defines the inverse core transform $\mathbf{C}_{i 4}(2.2)$ that decoders must implement. The forward core transform $\mathbf{C}_{f 4}(2.3)$ is derived but the product of the two core transforms results in an non-identity matrix. This discrepancy is corrected by another matrix $\mathbf{M}_{f}$, which does scaling and quantization at the same time. The matrix $\mathbf{M}_{f}$ is selected from the QP setting. The matrix $\mathbf{M}_{f}$ is actually a uniform scalar quantizer, which the standard defines as having 52 levels and indexed by a $Q_{s t e p}$ value. The step size of the quantizer doubles for every $\operatorname{sixth} Q_{\text {step }}$. The relation between $Q_{\text {step }}$ and QP is as follows [15]:

$$
Q_{s t e p}(\mathrm{QP})=Q_{\text {step }}(\mathrm{QP} \% 6) \cdot 2^{\mathrm{floor}(\mathrm{QP} / 6)}
$$

Logically, the quantisation equation (2.5) is applied to the coefficients outputted by the core transform is

$$
l_{i, j}=\operatorname{sign}\left(\theta_{i, j}\right)\left\lfloor\frac{\left|\theta_{i, j}\right| \alpha_{i, j}\left(Q_{s t e p}\right)}{Q_{s t e p}}\right\rfloor
$$

In practice, encoders do away with division and use shift operations. H.264/AVC standard tries to perform most of the computation using integers or fixed-point arithmetic to eliminate rounding errors. Also, integrating a normalization step within the quantization process, minimizes the number of multiplications required to process a block of image or residual data.

\subsubsection{Developing the Forward Transform and Quantization Process}

This section will provide the complete forward transform, quantization and scaling process for $4 \times 4$ blocks. The section will not derive every equation and matrix. 
Papers by Malvar et al [19] [3] or the book by Richardson [15] and the standard itself [1] [2] presents more comprehensive coverage of this subject. The purpose of this section is to give a general overview of the forward transform process employed to create H.264/AVC frames from raw greyscale images that used in our experiments. First, the quantization and scaling process is carried out by creating the matrix $\mathbf{M}_{f 4}$ which will be covered in the subsequent subsection. The last subsection will display the complete forward transform and quantization process.

\section{Developing Quantization Matrix $\mathbf{M}_{f 4}$}

Here we will show how to develop the quantization and scaling matrix $\mathbf{M}_{f 4}$. First, create a uniform scalar quantizer array $Q_{\text {step }}$ with 52 levels as follows:

$$
Q_{\text {step }}[n]=64 \cdot 0.625 \cdot 2^{n / 6}
$$

where every sixth step $n$, the values in array $Q_{\text {step }}$ will double.

Select quantization parameter $Q P$ between the values of 0 to 52 . The higher the $Q P$ value the greater the distortion. Then create the matrix $\mathbf{V}_{i 4}$ by multiplying matrix $\mathbf{S}_{i 4}$ in Equation (2.8) with the value stored in the array $Q_{\text {step }}$ at index $Q P$ :

$$
\mathbf{V}_{i 4}=\mathbf{S}_{i 4} \cdot Q_{s t e p}[Q P]
$$

where $\mathbf{S}_{i 4}$ is defined as

$$
\mathbf{S}_{i 4}=\left[\begin{array}{cccc}
1 / 4 & 1 / \sqrt{10} & 1 / 4 & 1 / \sqrt{10} \\
1 / \sqrt{10} & 2 / 5 & 1 / \sqrt{10} & 2 / 5 \\
1 / 4 & 1 / 2 \sqrt{10} & 1 / 4 & 1 / 2 \sqrt{10} \\
1 / \sqrt{10} & 2 / 5 & 1 / \sqrt{10} & 2 / 5
\end{array}\right]
$$

Using $\mathbf{V}_{i 4}$ we can now derive $\mathbf{M}_{\mathbf{f} 4}$ as follows:

$$
\mathbf{M}_{f 4}=\operatorname{round}\left(\frac{\mathbf{S}_{i 4} \bullet \mathbf{S}_{f 4} \cdot 2^{21}}{\mathbf{V}_{i 4}}\right)
$$

The operator • denotes an element-by-element multiplication, the Hadamard or Schur product, where $\mathbf{P}=\mathbf{Q} \bullet \mathbf{R}$ means each element $p_{i j}=q_{i j} \cdot r_{i j}$. The matrix $\mathbf{S}_{f 4}$ is defined below in Equation (2.10). During implementation both matrix $\mathbf{S}_{i 4}$ and $\mathbf{S}_{f 4}$ 
were defined once as a static constant variables.

$$
\mathbf{S}_{f 4}=\left[\begin{array}{cccc}
1 / 4 & 1 / 2 \sqrt{10} & 1 / 4 & 1 / 2 \sqrt{10} \\
1 / 2 \sqrt{10} & 1 / 10 & 1 / 2 \sqrt{10} & 1 / 10 \\
1 / 4 & 1 / 2 \sqrt{10} & 1 / 4 & 1 / 2 \sqrt{10} \\
1 / 2 \sqrt{10} & 1 / 10 & 1 / 2 \sqrt{10} & 1 / 10
\end{array}\right]
$$

\section{The Complete $4 \times 4$ Forward Transform with Quantization and Scaling}

\section{Process}

The complete $4 \times 4$ forward transform with quantization and scaling process becomes:

$$
\mathbf{Y}=\operatorname{round}\left(\mathbf{C}_{f 4} \cdot \mathbf{X} \cdot \mathbf{C}_{f 4}^{T} \cdot \mathbf{M}_{f 4} \cdot \frac{1}{2^{15}}\right)
$$

The final constant term of $1 / 2^{15}$ scales the quantization process. The standard body chose this value as a compromise between higher accuracy and limited arithmetic precision. Next we will look at the inverse transform with dequantization process.

\subsubsection{Developing the Inverse Transform Process}

This section outlines how to develop rescaling and inverse quantization operation followed by a two-dimensional inverse transform process. In the previous section, we discuss how to create the matrix $\mathbf{M}_{f 4}$ for the forward transform process using the matrix $\mathbf{V}_{i 4}$. This matrix is used in the inverse transform process to rescale and dequantize the matrix $\mathbf{Y}$ before inverse transform is applied. Therefore, the complete rescaling and inverse quantization followed by transform process for $4 \times 4$ blocks in a marcroblock is a follows:

$$
\mathbf{Z}=\operatorname{round}\left(\mathbf{C}_{i 4}^{T} \cdot \mathbf{Y} \bullet \mathbf{V}_{i 4} \cdot \mathbf{C}_{i 4} \cdot \frac{1}{2^{6}}\right)
$$

The standard body selected the rounding and constant term $1 / 2^{6}$ to compensate for the inverse quantization process.

\subsection{Performance Measurements}

While developing resizing algorithms in the compressed domain, analyzing the costbenefit of the algorithm compared to resizing in the spatial domain should be considered. In this regard, the thesis employed two performance measurements: image 
quality and computational cost. For measuring image quality, peak signal-to-noise ratio, often abbreviated as PSNR, was used to measure the distortion between resized image with the original or image resized in the spatial domain. For computational cost, we used a model first described by Mukherjee and Mitra [13] that calculates the cost associated with matrix operations. This section will first cover PSNR followed a subsection on computational cost model.

\subsubsection{Peak Signal-to-Noise Ratio (PSNR)}

The image quality measurement peak signal-to-noise ratio, often abbreviated as PSNR, is the ratio of the maximum possible power of signal and the power of the distortion that affects the fidelity of the reconstructed representation. The PSNR is a popular measure of quality used to judge reconstruction of images from lossy compression codecs. The signal in this case is the original image and the noise or distortion is the error introduced by compression technique. In this thesis, the signal is the original image and the noise is the error introduced by resizing algorithm on the original image.

It is easier to define PSNR via mean square error (MSE). Let $I$ and $K$ be two monochrome images of size $m \times n$, where one is the original and the other is the resized image, which is the noisy approximation of the original. Then MSE is defined as:

$$
M S E=\frac{1}{m n} \sum_{i=0}^{m-1} \sum_{j=0}^{n-1}[I(i, j)-K(i, j)]^{2}
$$

Then PSNR can be defined as:

$$
\begin{aligned}
P S N R & =10 \cdot \log _{10}\left(\frac{M A X_{I}^{2}}{M S E}\right) \\
& =20 \cdot \log _{10}\left(\frac{M A X_{I}}{\sqrt{M S E}}\right) \\
& =20 \cdot \log _{10}\left(M A X_{I}\right)-10 \cdot \log _{10}(M S E)
\end{aligned}
$$

Where $M A X_{I}$ is the maximum possible pixel value of the image. In this thesis, grey scale images were used where each pixel is represented using 8 bits, which provides 255 possible values to express all the shades of grey between white to black inclusive. 
Notice PSNR expressed distortion in terms of the logarithmic decibel (dB) scale. Typical values for PSNR in lossy image and video compression are between 30 and 50 $\mathrm{dB}$, where higher is better. In wireless networks, PSNR values about 20 to $25 \mathrm{~dB}$ are considered acceptable. Two images are identical if the MSE is equal to zero, which makes PSNR value undefined.

PSNR is an objective measurement of image quality in respect to a reference image and is approximation to human visual system. Therefore, people will subjectively judge some reconstruction image quality to be closer to the original image even though it has a lower PSNR value. This is one of the disadvantages of using PSNR for measuring image quality. However, in most cases PSNR provides reasonable objective measurement for image quality that is closely matches what a human would observe and report.

\subsubsection{Computational Cost}

It would be more desirable to resize video directly in the compressed format, because it offers saving in computation and memory cost compared to equivalent operations in the spatial domain. To measure this cost we needed a method that is independent of a platform's processing capabilities. Most of the computational cost with these resize algorithms is associated with matrix operations. Matrices that are sparse ${ }^{1}$ or containing elements equal to 1 or power of 2 can be exploited. For example, if a matrix entry is 1 or 0 we can ignore counting the number of multiplications when computing cost. Similarly, elements expressed in integral powers of 2 can be carried out using fixpoint arithmetic where multiplication or division operations are performed by binary left or right shift operations, hence do not count when computing cost.

Mukherjee and Mitra [13] created a model to calculate computational cost in terms the number of multiplication and additions. Let matrix $A$ of size $L \times N$ be multiplied with another matrix $B$ of size $N \times M$. Let $z_{i}$ be the number of zero elements and $d_{i}$ be the number of distinct elements (of distinct magnitude only) in the $i$ th row of $A$. Therefore, the total number of multiplications $\left(n_{m}().\right)$ and the total number of

\footnotetext{
${ }^{1}$ In this thesis, sparse matrix is defined as a matrix containing enough zero entries to contribute in reducing computational cost. Another popular definition is a matrix containing mostly zero entries.
} 
additions $\left(n_{a}().\right)$ are given by the following equations:

$$
\begin{aligned}
n_{m}(A ; L, N, M) & =M \sum_{i=1}^{L} d_{i} \\
n_{a}(A ; L, N, M) & =M \sum_{i=1}^{L}\left(N-z_{i}-1\right)
\end{aligned}
$$

Mukhopadhyay [14] referred to this model as the general computational complexity model of matrix multiplication (GCCMMM). In this model, the matrix $B$ is ignored and is considered as a variable such as input block of coefficients, while the $A$ is a fixed matrix. In practice, $B$ can be sparse. Encoding an H.264/AVC frame with a high QP value will cause most of the DCT coefficients to be zero. Hence, GCCMMM computes the upper limits of matrix operations for a given matrix $A$. In the next section, we will apply this model to compute the computational cost of resizing an H.264/AVC frame in the spatial domain.

\subsection{Resizing an Image in the Spatial Domain}

Before discussing resizing images in the compressed domain, a brief description of a simple and straight forward approach of resizing an image in the spatial domain using a bilinear interpolation method is presented. Let $c_{1}, c_{2}, c_{3}, c_{4}$ denote four adjacent $8 \times 8$ blocks in the spatial domain that form a $2 \times 2$ block as shown in Figure 2.3. Then spatial downsampling averages each $2 \times 2$ block (each containing a $8 \times 8$ block of DCT coefficients) to get a downsample $8 \times 8$ block $c$ as shown in Figure 2.3. The downsizing operation can be represented using matrix notation as follows [12][7][11]:

$$
c=\sum_{i=1}^{4} h_{i} c_{i} g_{i}
$$

where the dowsampling filters $h_{i}, g_{i}$ are given by

$$
\begin{aligned}
& h_{2}=g_{1}^{t}=g_{3}^{t}=h_{1}=\left[\begin{array}{l}
\mathbf{u}_{4 \times 8} \\
\mathbf{o}_{4 \times 8}
\end{array}\right] \\
& h_{4}=g_{2}^{t}=g_{4}^{t}=h_{3}=\left[\begin{array}{l}
\mathbf{o}_{4 \times 8} \\
\mathbf{u}_{4 \times 8}
\end{array}\right]
\end{aligned}
$$




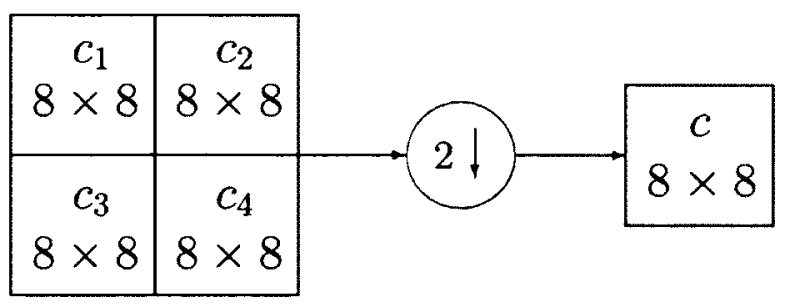

Figure 2.3: Downsampling four adjacent $8 \times 8$ DCT blocks by a factor of two to get a single $8 \times 8$ DCT block in the spatial domain.

where $\mathbf{u}_{4 \times 8}$ is defined in Equation (2.20) and $\mathbf{o}_{4 \times 8}$ is a $4 \times 8$ zero matrix.

$$
\mathbf{u}_{4 \times 8}=\left[\begin{array}{cccccccc}
0.5 & 0.5 & 0 & 0 & 0 & 0 & 0 & 0 \\
0 & 0 & 0.5 & 0.5 & 0 & 0 & 0 & 0 \\
0 & 0 & 0 & 0 & 0.5 & 0.5 & 0 & 0 \\
0 & 0 & 0 & 0 & 0 & 0 & 0.5 & 0.5
\end{array}\right]
$$

However, the process of decompressing, spatial domain downsizing and recompressing the video bitstream can be computationally too expensive for most real-time application. Carrying out the resizing operation directly in the compressed domain would avoid the high computational overhead associated with decompression and compression operations.

\subsubsection{Computational Cost when Halving an H.264/AVC Frame in the Spatial Domain}

Using the straight forward approach that was presented earlier, we will determine the computational cost for halving a H.264/AVC frame in the spatial domain. We will refer to this cost later in thesis when comparing with other halving and doubling algorithms. Let $c_{1}, c_{2}, c_{3}, c_{4}$ represent four blocks of $4 \times 4$ DCT coefficients that form a $2 \times 2$ block. Steps for downsampling an H.264/AVC frame in the spatial domain are: 1) inverse transform on four blocks of $4 \times 4$ DCT coefficients; 2) spatial domain resizing; 3) forward transform of the one downsampled $4 \times 4$ spatial block to one block containing $4 \times 4$ DCT coefficients. The computational cost will be measured 
by the number of multiplications $(M)$ and number of additions $(A)$ per pixel of the original image. The following is computational cost of halving an image in the spatial domain.

- Inverse transform on four $4 \times 4$ blocks of DCT coefficients (includes quantization and scaling): $(4 \times 16 M)+(8 \times 48 A)$. Applying the scaling and quantization matrix $\mathbf{V}_{i 4}$ on $c_{i}$ is an element-by-element multiplication which cost $16 M$. Following this stage, multiplying with core inverse transform $\mathbf{C}_{i 4}$ which cost $48 \mathrm{~A}$ but $0 M$ because core transform matrix's elements are $1,-1,1 / 2$, and $-1 / 2$. Elements equal to $1 / 2$ or $-1 / 2$ can be implemented using right bitwise shift operation so we do not count them. Multiplying the scaling constant $1 / 2^{6}$ can be implemented as a bitwise shift operation so it is not counted. We multiply the $\mathrm{C}_{i 4}$ twice for each input block for a total of $8 \times 48 A=384 A$.

- Resizing: We are averaging three additions per four pixels, hence $3 \times 4 \times 4 / 4=$ $48 \mathrm{~A}$. The resizing matrix is integral power of 2 , which can be implemented as a bitwise shift operation so we do not count them.

- Forward transform on one $4 \times 4$ block of pixels (includes quantization and scaling): $16 M+(2 \times 48 A)$. Similar to the inverse transform, the core transform $\mathbf{C}_{f 4}$ contains elements that we do not count for multiplication. It cost $16 M$ when the scaling and quantization matrix $\mathbf{M}_{f 4}$ after the transform stage. Multiplying the scaling constant $1 / 2^{15}$ can be implemented as a bitwise shift operation so it is not counted.

- Total of: $80 M+528 A$.

- Total per pixel of the original $8 \times 8$ frame (four $4 \times 4$ blocks of DCT coefficients): 64 pixels.

- Operations per pixel of the original image $(80 M / 64+528 A / 64): 1.25 M+8.25 A$.

Resizing H.264/AVC frames in the spatial domain can be very efficient when using integer transforms. Dugad and Ahuja [12] calculated spatial domain resizing to be $3.4375 M+9.8125 A$ when resizing an image containing blocks of $8 \times 8$ DCT coefficients and using a floating-point based $8 \times 8$ DCT. Therefore, resizing H.264/AVC frames 
in the spatial domain has a lower computational cost than previous standards that employed floating-point $8 \times 8 \mathrm{DCT}$.

\subsection{Subband DCT Computation}

DCT de-correlates the data being transformed so most of the energy is stored in a few transform coefficients. To achieve a high compression ratio, encoders will discard high frequency coefficients through quantization and leave the lower frequency coefficients in the transform blocks. Even though the high frequency coefficients are missing during the decoding process, the distortion displayed in the decoded image is small.

Subbands are components in a sequence. Using subband DCT computation one can extract coefficients from the sequence and discard the rest. A brief presentation of one dimension subband DCT computation will be first presented before discussing $N \times N$ point DCT. The DCT of a $N$ point data sequence $x(n), n=0,1,2, \ldots N-1$ is defined as

$$
\begin{aligned}
C(k)= & \sum_{n=0}^{N-1} 2 x(n) \cos \left(\frac{(2 n-1) \pi k}{2 N}\right) \\
& 0 \leq k \leq N-1
\end{aligned}
$$

Decompose a $N$ length input sequence $x(n)$ with $N$ being even, into two subsquence $x_{L}(n)$ and $x_{H}(n)$ of length $N / 2$ each

$$
\begin{aligned}
x_{L}(n)= & \frac{1}{2}\{x(2 n)+x(2 n+1)\}, \\
x_{H}(n)= & \frac{1}{2}\{x(2 n)-x(2 n+1)\}, \\
& n=0,1, \ldots, \frac{N}{2}-1 .
\end{aligned}
$$

For brevity the following is the relationship between the DCT of subbands (of $\frac{N}{2}$ points) from Equation 2.22 with the original DCT (of $N$-points) of the sequence from Equation 2.21. A more detail explanation can be found in [20][21][14]. Let $C_{L}(k)$ be the $N / 2$-point DCT of $x_{L}(n)$ and $S_{H}(k)$ be the $N / 2$-point DST of $x_{H}(n)$. Then the computation of DCT of $x(n)$ from $C_{L}(k)$ 's and $S_{H}(k)$ 's could be performed as follows $[20]:$

$$
\begin{aligned}
C(k)= & 2 \cos \left(\frac{\pi k}{2 N}\right) \overline{C_{L}}(k)+2 \sin \left(\frac{\pi k}{2 N}\right) \overline{S_{H}}(k) \\
& 0 \leq k \leq N-1
\end{aligned}
$$


where

$$
\overline{C_{L}}(k)= \begin{cases}C_{L}(k) & : 0 \leq k \leq \frac{N}{2}-1 \\ 0 & : k=\frac{N}{2} \\ -C_{L}(N-k) & : \frac{N}{2}+1 \leq k \leq N-1\end{cases}
$$

and

$$
\overline{S_{L}}(k)= \begin{cases}S_{H}(k) & : 0 \leq k \leq \frac{N}{2}-1 \\ 2 \sum_{n=0}^{N / 2-1}(-1)^{n} x_{H}(n) & : k=\frac{N}{2} \\ S_{H}(N-k) & : \frac{N}{2}+1 \leq k \leq N-1 .\end{cases}
$$

with $C_{L}(k)$ representing the $(N / 2)$-point DCT of $x_{L}(n)$, and $S_{H}(k)$ representing the $(N / 2)$-point discrete sine transform (DST) of $x_{H}(n)$. The computation of the $N$-point DCT using 2.23 requires the computation of an $(N / 2)$-point DCT and an $(N / 2)$-point DST is called subband DCT [20].

\subsubsection{Approximate DCT Computation}

In most practical applications, most of the energy of a signal is concentrated in the lower-frequency zone. Assume that most of the signal is in the frequency range $k \in\{0,1, \ldots, N / 2-1\}$ then Equation 2.23 can be approximated as [20] [21]

$$
C(k)= \begin{cases}2 \cos \left(\frac{\pi k}{2 N}\right) \overline{C_{L}}(k) & : k \in\left\{0,1, \ldots, \frac{N}{2}-1\right\} \\ 0 & : \text { otherwise. }\end{cases}
$$

This approximation is referred to as the subband approximation of DCT. It was noted that for small values of $k$, cosine terms can be approximated as 1 . Therefore, Equation 2.26 can be further simplified to

$$
C(k)= \begin{cases}2 \overline{C_{L}}(k) & : \quad k \in\left\{0,1, \ldots, \frac{N}{2}-1\right\} \\ 0 & : \text { otherwise. }\end{cases}
$$

This simplified approximation is referred to as the low-pass truncated approximation of DCT.

\subsubsection{Approximate DCT in 2-D}

In practical applications in image processing, the underlying assumption is almost all images contain mostly very low-frequency components. Extending $(N)$-point DCT 
approximation Equation 2.26 and 2.27 to obtain low-low $(L L)$ frequency of a 2-D DCT image is as follows [20] [21]. Let $\overline{C_{L L}}(k, l), 0 \leq k, l \leq(N / 2)$ be the 2-D DCT of $x_{L L}(m, n)$. Then the subband approximation of DCT of $x(m, n)$ is given by

$$
C(k, l)= \begin{cases}2 \cos \left(\frac{\pi k}{2 N}\right) \cos \left(\frac{\pi l}{2 N}\right) \overline{C_{L L}}(k, l) & : \quad k, l \in\left\{0,1, \ldots, \frac{N}{2}-1\right\} \\ 0 & : \text { otherwise. }\end{cases}
$$

As in the $(N)$-point DCT case, Equation 2.28 can further simplified to

$$
C(k, l)= \begin{cases}2 \overline{C_{L L}}(k, l) & : \quad k, l \in\left\{0,1, \ldots, \frac{N}{2}-1\right\} \\ 0 & : \text { otherwise. }\end{cases}
$$

The above equation is referred as the low-pass truncated approximation of the subband coefficients.

\subsection{Composition and Decomposition of Blocks of DCT Coefficients}

The composition and decomposition of blocks of DCT coefficients was proposed by Jiang and Feng [22] on the spatial relationship of DCT coefficients between a block and its sub-blocks. Their paper outlined how one can transform blocks of DCT coefficients from one vector space to another vector space. For example, the authors explored potential applications where one can compose $16 \times 16$ blocks of DCT coefficients from $8 \times 8$ blocks. They also provided an example of the opposite where one can decompose $8 \times 8$ blocks of DCT coefficients to $4 \times 4$ sub-blocks. We will later show how composition and decomposition are used in several DCT domain resizing algorithms.

To understand composition and decomposition, we will first look at the spatial relationship of DCT blocks in one dimension. Let $x(n), n=0,1, \ldots, M N-1$ be a sequence of length $M N$. The sequence can be partitioned into $M$ blocks each containing $N$ data points. In block DCT space, an $N$-point DCT is applied to each block of $N$ data points. Therefore, the $N$-point DCT of the $p$ th block can be represented as follows:

$$
\begin{aligned}
C_{p}(k)= & \sqrt{\frac{2}{N}} \alpha(k) \sum_{n=0}^{N-1} x(p N+n) \cos \left(\frac{(2 n+1)}{2 N}\right) \\
& 0 \leq p \leq M-1,0 \leq k \leq N-1
\end{aligned}
$$


On the other hand, the $M N$-point DCT of $x(n)$ is given

$$
\begin{aligned}
C(k)= & \sqrt{\frac{2}{M \times N}} \alpha(k) \sum_{n=0}^{M \times N-1} x(n) \cos \left(\frac{(2 n+1) \pi k}{2 \times M \times N}\right), \\
& 0 \leq k \leq M \times N-1
\end{aligned}
$$

Jiang and Feng recognized that a block DCT transformation as outlined in Equation (2.30)is an orthonormal expansion of the sequence $\{x(n)\}$ with a set of $M N$ basis vectors, where each is derived from the basis vectors on the $N$-point DCT. Hence, there exists an invertible linear transformation or mapping from $M$ blocks of the $N$-point DCT transform to the $N M$-point DCT transform. This implied that for a sequence of $N$-point DCT blocks $\left\{C_{i}^{(N)}\right\}, i=0,1, \ldots, M-1$, there is a matrix $\mathbf{A}_{(M, N)}$ of size $M N \times M N$ such that the corresponding composite DCT $\mathbf{C}^{(M N)}$ holds the following relation with $M$ block DCTs:

$$
\mathbf{C}^{(M N)}=\mathbf{A}_{(M, N)}\left[\mathbf{C}_{0}^{(N)} \mathbf{C}_{1}^{(N)} \ldots \mathbf{C}_{M-1}^{(N)}\right]^{T}
$$

where the superscript $T$ denotes the matrix transposition.

We can extend this analysis into two dimensions. For $L \times M$ adjacent DCT blocks, a composite DCT block can be formed as

$$
\mathbf{C}^{(L N \times M N)}=\mathbf{A}_{(L, N)}\left[\begin{array}{cccc}
\mathbf{C}_{0,0}^{(N \times N)} & \mathbf{C}_{0,1}^{(N \times N)} & \cdots & \mathbf{C}_{0, M-1}^{(N \times N)} \\
\mathbf{C}_{1,0}^{(N \times N)} & \mathbf{C}_{1,1}^{(N \times N)} & \ldots & \mathbf{C}_{1, M-1}^{(N \times N)} \\
\ldots \ldots \cdots \cdots & \ldots \cdots \cdots & \ldots \cdots \cdots \\
\mathbf{C}_{L-1,0}^{(N \times N)} & \mathbf{C}_{L-1,1}^{(N \times N)} & \cdots & \mathbf{C}_{L-1, M-1}^{(N \times N)}
\end{array}\right] \mathbf{A}_{(M, N)}^{T}
$$

To decompose a DCT block $\mathrm{C}^{(L N \times M N)}$ to $L \times M$ DCT blocks of size $N \times N$ each, the following expression is used:

$$
\left[\begin{array}{cccc}
\mathbf{C}_{0,0}^{(N \times N)} & \mathbf{C}_{0,1}^{(N \times N)} & \cdots & \mathbf{C}_{0, M-1}^{(N \times N)} \\
\mathbf{C}_{1,0}^{(N \times N)} & \mathbf{C}_{1,1}^{(N \times N)} & \ldots & \mathbf{C}_{1, M-1}^{(N \times N)} \\
\ldots \ldots \ldots & \ldots \ldots \ldots & \ldots & \ldots \ldots \ldots \\
\cdots & \ldots \ldots & \ldots \\
\mathbf{C}_{L-1,0}^{(N \times N)} & \mathbf{C}_{L-1,1}^{(N \times N)} & \cdots & \mathbf{C}_{L-1, M-1}^{(N \times N)}
\end{array}\right]=\mathbf{A}_{(L, N)}^{-1} \mathbf{C}^{(L N \times M N)} \mathbf{A}_{(M, N)}^{-1^{T}}
$$

An example of a conversion matrix is provided below.

$$
\mathbf{A}_{(2,4)}=\mathbf{C}_{8}\left[\begin{array}{cc}
\mathbf{C}_{4}^{-1} & 0_{4} \\
0_{4} & \mathbf{C}_{4}^{-1}
\end{array}\right]
$$




$$
=\left[\begin{array}{cccccccc}
1 / \sqrt{2} & 0 & 0 & 0 & 1 / \sqrt{2} & 0 & 0 & 0 \\
0.6407 & 0.2940 & -0.0528 & 0.0162 & -0.6407 & 0.2940 & 0.0528 & 0.0162 \\
0 & 1 / \sqrt{2} & 0 & 0 & 0 & -1 / \sqrt{2} & 0 & 0 \\
-0.2250 & 0.5594 & 0.3629 & -0.0690 & 0.2250 & 0.5594 & -0.3629 & -0.0690 \\
0 & 0 & 1 / \sqrt{2} & 0 & 0 & 0 & 1 / \sqrt{2} & 0 \\
0.1503 & -0.2492 & 0.5432 & 0.3468 & -0.1503 & -0.2492 & -0.5432 & 0.3468 \\
0 & 0 & 0 & 1 / \sqrt{2} & 0 & 0 & 0 & -1 / \sqrt{2} \\
-0.1274 & 0.1964 & -0.2654 & 0.6122 & 0.1274 & 0.1964 & 0.2654 & 0.6122
\end{array}\right]
$$

The conversion matrix $\mathbf{A}_{(2,4)}$ contains several zero entries, which requires fewer numbers of multiplications and additions during matrix multiplications.

\subsection{Summary}

The chapter started with an introduction of the video compression standard, H.264/AVC. In this short overview of H.264/AVC, the section covered the major coding elements to construct a forward and inverse transform process. We next discussed performance measures: one for image quality using PSNR and one for computational cost using general computational complexity model of matrix multiplication (GCCMMM). Next, the chapter provided an example of resizing an H.264/AVC frame in the spatial domain. The number of multiplications and additions computed in this example will offer a benchmark when comparing with other resize algorithms. The chapter introduced two techniques that are part of several resize algorithms. The first technique was subband DCT computation which extracts the lower frequency coefficients from a block of DCT coefficients. The second technique was on composition and decomposition of DCT blocks. Applying this technique, one can composed $16 \times 16$ blocks of DCT coefficients from $8 \times 8$ blocks or decomposed them in the inverse direction. The next chapter will introduce the state of the art of image resizing in the compressed domain. 


\section{Chapter 3}

\section{DCT Domain Image Resizing Algorithms}

\subsection{Introduction}

In the early days of developing resizing algorithms in the transform domain, researchers devoted effort to image halving and doubling problem. Some of the early contributors in this field were Chang and Messerchmitt [7], and Merhav and Bhaskaran [11] who developed resizing algorithms that exploit linear, distributive and unitary transform properties of DCT. Although image quality was similar and often times superior than resizing in the spatial domain, the computational cost was almost same as spatial domain resizing techniques.

Dugad and Ahuja [12] developed a simple fast computation algorithm for image halving and doubling by exploiting the low frequency DCT coefficients. Later, Mukherjee and Mitra [21] proposed modifications to Dugad and Ahuja [12] schema. These modifications improved image quality but increased computational cost. Mukherjee and Mitra [21] observed both of these techniques use subband approximation of DCT coefficients while performing image resizing operations in the frequency domain.

Jiang and Feng [22] formulated spatial relationships of the DCT coefficients between a block and sub-blocks. Using their approach one can decompose and recompose the blocks of DCT coefficients. Mukherjee and Mitra [13] created several resizing algorithms based on decomposition and re-composition combined with subband approximation. Depending on the ordering of these operations, can vary the computational cost and the image quality.

Resizing images by arbitrary factors is still active area of research. Salazar and Tran [5][23] extended the technique developed by Dugad and Ahuja [12]. They proposed a single mapping that implicitly involves a combined resizing and inverse transform back into the spatial domain followed by a combined resizing and forward transform into the DCT domain. The algorithm resizes at both the inverse and forward transformations, which allows images to be resize at different scaling factors $S=I / O$ 
other than power of two. One can use different $N$-point inverse and $M$-point forward DCTs of the original image can be used to vary the final image quality for a given scaling factor $S=O / I$. However, their algorithm maintains the aspect ratios of the downsampled or upsampled image.

Mukhopadhyay [14] proposed a hybrid approach for arbitrary resizing, which means resizing operation cannot be expressed as a downsampling or upsampling operation. His hybrid resizing algorithm extended the approach developed by Mukherjee and Mitra [13]. The algorithm follows computational sequence of block composition, subband approximation and block decomposition. However, the subband approximation relationship is put into a more general form. For example, this allows applications to resize HDTV frame $(1080 \times 1920)$ to an NTSC frame $(480 \times 640)$.

This chapter will cover thirteen image resize algorithms that work in the DCT domain. All these algorithm are based on two basic assumptions. The researchers assumed that the image is compressed using $8 \times 8$ DCT block framework and inverse quantization was applied on the DCT coefficients before resizing.

\subsection{Image Halving}

Image Halving is an operation that takes an image of size $N \times N$ and outputs an image of $N / 2 \times N / 2$. The following subsection outlines image halving algorithms that operated on compressed images that are composed on $8 \times 8$ DCT blocks.

\subsubsection{Image Halving through Bilinear Interpolation (IHBI)}

The basic idea of this approach is to perform the resizing operation using the straight forward technique outlined in Equation (2.18) in the DCT domain that was presented in Section 2.4. Let DCT $(c)$ denote the DCT block coefficients of $8 \times 8$ block $c$ in Equation (2.18). In this approach, obtain $\operatorname{DCT}(c)$ from the given $\operatorname{DCT}\left(c_{i}\right)$ for $i=1$ to 4 . The approach depends on the fact that the DCT, being a linear orthonormal transform, is distributive over matrix multiplication [12], [7],[11]. Let $T$ denote the $8 \times 8$ DCT operator matrix. Since $T T^{t}=T^{t} T=I$, then

$$
\operatorname{DCT}(c)=T c T^{t}=\sum_{i=1}^{4} \operatorname{DCT}\left(h_{i}\right) \operatorname{DCT}\left(c_{i}\right) \operatorname{DCT}\left(g_{i}\right)
$$


The matrices DCT $\left(h_{i}\right)$ and $\operatorname{DCT}\left(g_{i}\right)$ for $i=1$ to 4 can be precomputed and stored. During runtime, given $\operatorname{DCT}\left(c_{i}\right)$ for $i=1$ to 4 , compute using matrix multiplication to obtain $\operatorname{DCT}(c)$.

Even though the filter matrix $h_{i}$ and $g_{i}$ are sparse, the $\operatorname{DCT}\left(h_{i}\right)$ and $\operatorname{DCT}\left(g_{i}\right)$ are not sparse at all. Therefore, the matrix multiplication in Equation (3.1) can have complexity comparable to downsampling in the spatial domain [12].

\subsubsection{Image Halving through Low-Pass Filtering followed by Downsampling (IHFDS)}

Rakesh Dugad and Narendra Ahuja [12] developed an efficient image halving algorithm, which exploits the observation that $\operatorname{DCT}\left(c_{i}\right)$ for $i=1$ to 4 are mostly sparse (i.e. contains coefficients with the value equal to zero). They also noticed that zeroing the high frequency coefficients in a $8 \times 8$ DCT block has a small effect on final image quality.

Their approach views image halving as a low-pass filtering followed by downsampling, which is implemented in the DCT domain. The low-pass filter is chosen independent of the DCT transform. They designed the low-pass filter so that the DCT of the filter matrix is sparse instead of the filter matrix itself, hence providing computational savings. Then they combined the low-filter and the downsampling matrix together and applied it directly in the DCT domain.

Figure (3.1) shows a schematic representation of their approach. The figure shows a simple DCT scaling to downsize an image by a factor of two. Even though the final mapping operates entirely in the DCT domain it implicitly involves a transformation into the spatial domain while simultaneously resizing the image followed by a standard transformation back into the $N \times N$ block DCT domain.

Let $\mathbf{b}_{1}, \mathbf{b}_{2}, \mathbf{b}_{3}$, and $\mathbf{b}_{4}$ denote four consecutive $8 \times 8$ blocks in the spatial domain. Let $\mathbf{B}_{1}, \mathbf{B}_{2}, \mathbf{B}_{3}$, and $\mathbf{B}_{4}$ denote the $8 \times 8$ DCT blocks of these blocks. Let $\hat{\mathbf{B}}_{1}, \hat{\mathbf{B}}_{2}, \hat{\mathbf{B}}_{3}$, and $\hat{\mathbf{B}}_{4}$ represent the $4 \times 4$ matrices containing the low-pass coefficients of $\mathbf{B}_{1}, \mathbf{B}_{2}, \mathbf{B}_{3}$, and $\mathbf{B}_{4}$, respectively. Let $\hat{\mathbf{b}}_{1}, \hat{\mathbf{b}}_{2}, \hat{\mathbf{b}}_{3}$, and $\hat{\mathbf{b}}_{4}$ denote the $4 \times 4$ inverse DCT of $\hat{\mathbf{B}}_{1}, \hat{\mathbf{B}}_{2}, \hat{\mathbf{B}}_{3}$, and $\hat{\mathbf{B}}_{4}$, respectively. Then $\hat{\mathbf{b}} \stackrel{\text { def }}{=}\left[\begin{array}{ll}\hat{\mathbf{b}}_{1} & \hat{\mathbf{b}}_{2} \\ \hat{\mathbf{b}}_{3} & \hat{\mathbf{b}}_{4}\end{array}\right]$ is the low-pass and downscaled version of 


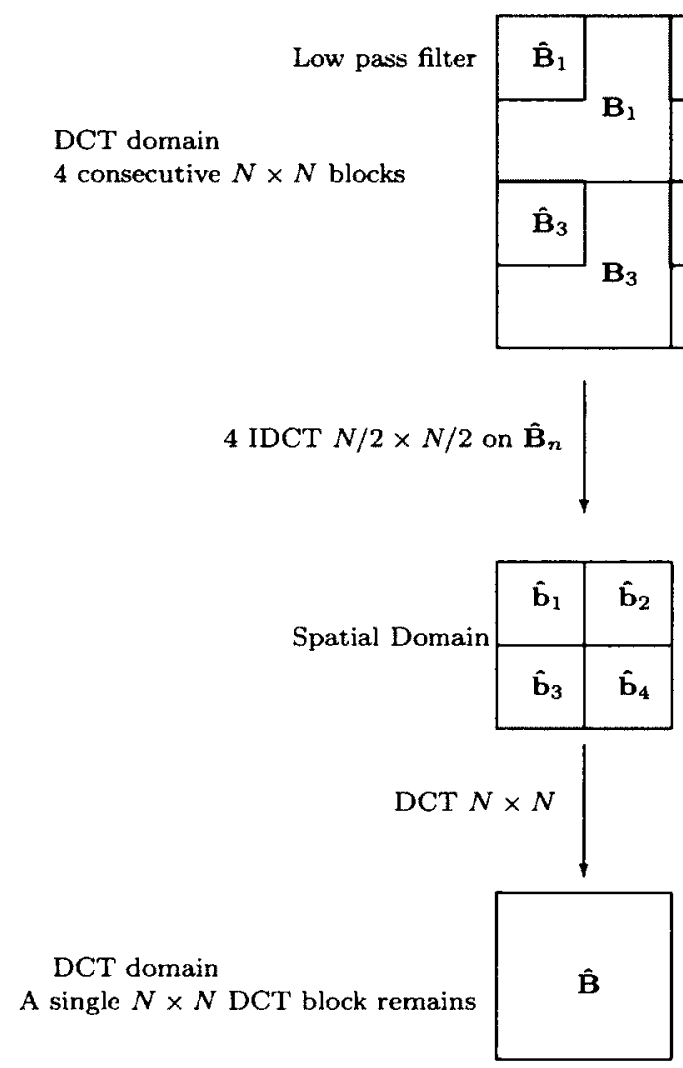

Figure 3.1: Schematic of the low-pass filtering followed by downsampling approach developed by Dugad and Ahuja [12] 
$\mathbf{b} \stackrel{\text { def }}{=}\left[\begin{array}{ll}\mathbf{b}_{1} & \mathbf{b}_{2} \\ \mathbf{b}_{3} & \mathbf{b}_{4}\end{array}\right]$. To compute $\hat{\mathbf{B}} \stackrel{\text { def }}{=} \mathrm{DCT}(\hat{\mathbf{b}})$ from $\mathbf{B}_{1}, \mathbf{B}_{2}, \mathbf{B}_{3}$, and $\mathbf{B}_{4}$, they outlined the following expression.

$$
\begin{aligned}
\hat{\mathbf{B}}= & T \hat{\mathbf{b}} T^{t} \\
= & {\left[T_{L} T_{R}\right]\left[\begin{array}{ll}
\hat{\mathbf{b}}_{1} & \hat{\mathbf{b}}_{2} \\
\hat{\mathbf{b}}_{3} & \hat{\mathbf{b}}_{4}
\end{array}\right]\left[\begin{array}{c}
T_{L}^{t} \\
T_{R}^{t}
\end{array}\right] } \\
= & {\left[T_{L} T_{R}\right]\left[\begin{array}{ll}
T_{4}^{t} \hat{\mathbf{B}}_{1} T_{4} & T_{4}^{t} \hat{\mathbf{B}}_{2} T_{4} \\
T_{4}^{t} \hat{\mathbf{B}}_{3} T_{4} & T_{4}^{t} \hat{\mathbf{B}}_{4} T_{4}
\end{array}\right]\left[\begin{array}{c}
T_{L}^{t} \\
T_{R}^{t}
\end{array}\right] } \\
= & \left(T_{L} T_{4}^{t}\right) \hat{\mathbf{B}}_{1}\left(T_{L} T_{4}^{t}\right)^{t}+\left(T_{L} T_{4}^{t}\right) \hat{\mathbf{B}}_{2}\left(T_{R} T_{4}^{t}\right)^{t} \\
& +\left(T_{R} T_{4}^{t}\right) \hat{\mathbf{B}}_{3}\left(T_{L} T_{4}^{t}\right)^{t}+\left(T_{R} T_{4}^{t}\right) \hat{\mathbf{B}}_{4}\left(T_{R} T_{4}^{t}\right)^{t}
\end{aligned}
$$

Dugad and Ahuja [12] proposed a decomposition method to convert Equation (3.5) further into a new form so that matrices become more sparse to reduce matrix operations.

\section{Low-Pass Filter Matrix}

As mentioned previously, the low-pass filter matrix can be defined independently to the downsampling matrices $T_{L} T_{4}^{t}$ and $T_{R} T_{4}^{t}$ outlined in Equation (3.5). The design of the filter matrix is such that the DCT of the filter matrix is sparse rather that the filter itself. This is desirable because it reduces computations in the compressed domain. Dugad and Ahuja [12] derived and defined the downsampling filter as

$$
M \stackrel{\text { def }}{=}\left[\begin{array}{l}
I \\
O
\end{array}\right]
$$

where $I$ denotes $4 \times 4$ identity matrix and $O$ denotes zero matrix. The authors further derived $\operatorname{DCT}\left(h_{1}\right)$ :

$$
\operatorname{DCT}\left(h_{1}\right)=T h T^{t}=\left[T_{L} T_{R}\right] M T_{4}^{t} M^{t} T T^{t}=T_{L} T_{4}^{t} M^{t}
$$

Multiplying $M^{t}$ at the end ensures that only the first $4 \times 4$ low-pass components of the DCT $\left(\mathbf{b}_{i}\right)$ are used in the computation of $\hat{\mathbf{b}}_{i}$. 


\subsubsection{Image Halving through Approximation followed by Composition (IHAC)}

Jayanta Mukherjee and Sanjit K. Mitra [13] developed image halving algorithm, which employs subband approximation and DCT composition. Let $b$ a $8 \times 8$ block in the spatial domain whose DCT coefficients are encoded as $8 \times 8$ block $\mathrm{B}$ in the compressed domain. In this algorithm, subband approximation are applied to four adjacent blocks B to obtain a decimated $4 \times 4$ blocks of DCT coefficients. Next, these $4 \times 4$ DCT blocks are merged using DCT composition to create a $8 \times 8$ block of DCT coefficients. Algorithm 1 describes how an image can be half using this approach. The algorithm developers [13] noted that this approach is similar to the algorithm developed by Dugad and Ahuja [12] where low-passed filter is applied first followed by downsampling. However, the computation cost is different.

Algorithm 1 IHAC [13]

Input: $8 \times 8$ block based DCT encoded image.

Output: $8 \times 8$ block based DCT encoded downsampled image.

for every four adjacent $8 \times 8, \mathbf{B}_{00}, \mathbf{B}_{01}, \mathbf{B}_{10}, \mathbf{B}_{11}$ of the input image do

Subband approximation: Get corresponding $4 \times 44$-point DCT blocks $\left\{\hat{\mathbf{B}}_{i j}^{(4 \times 4)}\right\}, i=0,1$ and $j=0,1$ using 2.28 or 2.29 .

Block composition: Convert four $4 \times 4$ DCT blocks to a $8 \times 8$ DCT block $\mathrm{B}_{d}$ as follows.

$$
\mathbf{B}_{d}=\mathbf{A}_{(2,4)}\left[\begin{array}{cc}
\hat{\mathbf{B}}_{00}^{(4 \times 4)} & \hat{\mathbf{B}}_{01}^{(4 \times 4)} \\
\hat{\mathbf{B}}_{10}^{(4 \times 4)} & \hat{\mathbf{B}}_{11}^{(4 \times 4)}
\end{array}\right] \mathbf{A}_{(2,4)}^{T}
$$

end for

The block composition matrix $\mathbf{A}_{(2,4)}$ used in the composition step is outlined as 
follows:

$$
\mathbf{A}_{(2,4)}=\left[\begin{array}{cccccccc}
1 / \sqrt{2} & 0 & 0 & 0 & 1 / \sqrt{2} & 0 & 0 & 0 \\
0.6407 & 0.2940 & -0.0528 & 0.0162 & -0.6407 & 0.2940 & 0.0528 & 0.0162 \\
0 & 1 / \sqrt{2} & 0 & 0 & 0 & -1 / \sqrt{2} & 0 & 0 \\
-0.2250 & 0.5594 & 0.3629 & -0.0690 & 0.2250 & 0.5594 & -0.3629 & -0.0690 \\
0 & 0 & 1 / \sqrt{2} & 0 & 0 & 0 & 1 / \sqrt{2} & 0 \\
0.1503 & -0.2492 & 0.5432 & 0.3468 & -0.1503 & -0.2492 & -0.5432 & 0.3468 \\
0 & 0 & 0 & 1 / \sqrt{2} & 0 & 0 & 0 & -1 / \sqrt{2} \\
-0.1274 & 0.1964 & -0.2654 & 0.6122 & 0.1274 & 0.1964 & 0.2654 & 0.6122
\end{array}\right]
$$

\subsubsection{Image Halving through Composition followed by Approximation (IHCA)}

Jayanta Mukherjee and Sanjit K. Mitra [13] developed a second approach for image halving, which employs subband approximation and DCT composition but in a different order than IHAC algorithm. In this approach, use block composition to merge four adjacent blocks of DCT coefficients $B$ to form a $16 \times 16$ block. Then apply subband approximation on this $16 \times 16$ block of DCT coefficients to obtain $8 \times 8$ block. Algorithm 2 describes how to use this approach for image halving.

Algorithm 2 IHCA [13]

Input: $8 \times 8$ block based DCT encoded image.

Output: $8 \times 8$ block based DCT encoded downsampled image.

for every four adjacent $8 \times 8, \mathbf{B}_{00}, \mathbf{B}_{01}, \mathbf{B}_{10}, \mathbf{B}_{11}$ of the input image do

Block composition: Convert four adjacent $8 \times 8$ DCT blocks to a $16 \times 16$ DCT block $\mathbf{B}^{(16 \times 16)}$ as follows.

$$
\mathbf{B}^{(16 \times 16)}=\mathbf{A}_{(2,8)}\left[\begin{array}{ll}
\mathbf{B}_{00} & \mathbf{B}_{01} \\
\mathbf{B}_{10} & \mathbf{B}_{11}
\end{array}\right] \mathbf{A}_{(2,8)}^{T}
$$

Subband approximation: Get the resulting $8 \times 8$ block using 2.28 or 2.29 . end for 


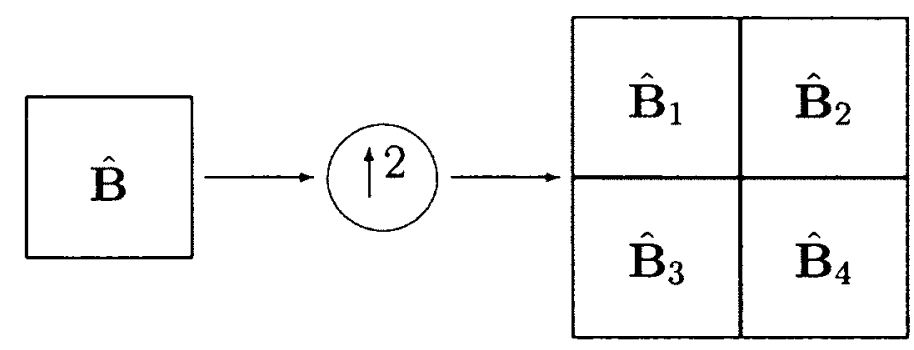

Figure 3.2: Image Doubling through Zero-Order Interpolation.

\subsection{Image Doubling}

There are applications where doubling an image from its downsampled version is required. As in the image halving case, it is desirable to have efficient algorithm in the compressed domain. Image doubling algorithms take an image of size $N \times N$ and output an image of size $2 N \times 2 N$. Basically, the following image doubling algorithms are the inverse of the image halving algorithms. The following subsection will cover four such algorithms.

\subsubsection{Image Doubling through Zero-Order Interpolation (IDZOI)}

Doubling an image's size using zero-order interpolation, Jayanta Mukherjee and Sanjit K. Mitra [13] followed a similar approach outlined in the halving case. The basic idea of this method is to read blocks of $8 \times 8 \mathrm{DCT}$ coefficients $\hat{\mathbf{B}}$ from a downsampled image and create four new $8 \times 8$ blocks of DCT coefficients, $\hat{\mathbf{B}}_{1}, \hat{\mathbf{B}}_{2}, \hat{\mathbf{B}}_{3}$ and $\hat{\mathbf{B}}_{4}$ for the upsampled image (See Figure 3.2).

Let $\hat{\mathbf{b}}=\operatorname{IDCT}(\hat{\mathbf{B}})$, which $\hat{\mathbf{b}}$ is the spatial representation of $\hat{\mathbf{B}}$. In the spatial domain, a simple and straight forward approach to derive the upsample blocks $\hat{\mathbf{b}}_{1}, \hat{\mathbf{b}}_{2}, \hat{\mathbf{b}}_{3}$ and $\hat{\mathbf{b}}_{4}$ can be represented using matrix notation as follows:

$$
\begin{aligned}
& \hat{\mathbf{b}}_{1}=g_{1} \hat{\mathbf{b}} h_{1} \\
& \hat{\mathbf{b}}_{2}=g_{1} \hat{\mathbf{b}} h_{2} \\
& \hat{\mathbf{b}}_{3}=g_{2} \hat{\mathbf{b}} h_{1} \\
& \hat{\mathbf{b}}_{4}=g_{2} \hat{\mathbf{b}} h_{2}
\end{aligned}
$$


where the upsampling filters, $h_{1}, h_{2}, g_{1}$ and $g_{2}$ are given by

$$
\begin{aligned}
& h_{1}=g_{1}^{t}=\left[\begin{array}{l}
\mathbf{u}_{4 \times 8} \\
\mathbf{o}_{4 \times 8}
\end{array}\right] \\
& h_{2}=g_{2}^{t}=\left[\begin{array}{l}
\mathbf{o}_{4 \times 8} \\
\mathbf{u}_{4 \times 8}
\end{array}\right]
\end{aligned}
$$

where $u_{4 \times 8}$ is defined in Equation (3.10) and $o_{4 \times 8}$ zero matrix.

$$
\mathbf{u}_{4 \times 8}=\left[\begin{array}{cccccccc}
1 & 1 & 0 & 0 & 0 & 0 & 0 & 0 \\
0 & 0 & 1 & 1 & 0 & 0 & 0 & 0 \\
0 & 0 & 0 & 0 & 1 & 1 & 0 & 0 \\
0 & 0 & 0 & 0 & 0 & 0 & 1 & 1
\end{array}\right]
$$

In the compressed domain, the straight forward approach expressed in Equation (3.8) becomes:

$$
\begin{aligned}
& \operatorname{DCT}\left(\hat{\mathbf{b}}_{1}\right)=\operatorname{DCT}\left(g_{1}\right) \operatorname{DCT}(\hat{\mathbf{b}}) \operatorname{DCT}\left(h_{1}\right) \\
& \operatorname{DCT}\left(\hat{\mathbf{b}}_{2}\right)=\operatorname{DCT}\left(g_{1}\right) \operatorname{DCT}(\hat{\mathbf{b}}) \operatorname{DCT}\left(h_{2}\right) \\
& \operatorname{DCT}\left(\hat{\mathbf{b}}_{3}\right)=\operatorname{DCT}\left(g_{2}\right) \operatorname{DCT}(\hat{\mathbf{b}}) \operatorname{DCT}\left(h_{1}\right) \\
& \operatorname{DCT}\left(\hat{\mathbf{b}}_{4}\right)=\operatorname{DCT}\left(g_{2}\right) \operatorname{DCT}(\hat{\mathbf{b}}) \operatorname{DCT}\left(h_{2}\right)
\end{aligned}
$$

where DCT is a regular forward DCT. Even though the upsampling matrix $h_{1}, h_{2}, g_{1}$ and $g_{2}$ are sparse, the forward DCT versions of them are not. This lack of spareness contributes to higher computational cost.

\subsubsection{Image Doubling through Upsampling followed by Zero Padding (IDUSZP)}

Rakesh Dugad and Narendra Ahuja [12] developed an efficient image doubling algorithm that is the inverse of the image halving algorithm, IHFDS, which was covered in the previous subsection on Image Halving.

For each $8 \times 8 \hat{\mathbf{B}}$ in the downsampled image, extract $\hat{\mathbf{b}}=\operatorname{IDCT}(\hat{\mathbf{B}})$. Partition $\hat{\mathbf{b}}$ into $2 \times 2$ sub-blocks so that

$$
\hat{\mathbf{b}}=\left[\begin{array}{ll}
\hat{\mathbf{b}}_{1} & \hat{\mathbf{b}}_{2} \\
\hat{\mathbf{b}}_{3} & \hat{\mathbf{b}}_{4}
\end{array}\right]
$$


Then compute $\hat{\mathbf{B}}_{1}=\operatorname{DCT}\left(\hat{\mathbf{b}}_{1}\right)$ and etc. Since $\hat{\mathbf{B}}_{1}$ contains all the low-frequency DCT coefficients of the corresponding $4 \times 4$ original image, create the following matrix

$$
\hat{\mathbf{B}}_{1}=\left[\begin{array}{cc}
\hat{\mathbf{B}} & \mathbf{O} \\
\mathbf{O} & \mathrm{O}
\end{array}\right]
$$

where $\mathbf{O}$ denotes a $4 \times 4$ zero matrix.

Therefore, $\hat{\mathbf{B}}_{1}$ DCT block corresponds to $\mathbf{B}_{1}$ DCT block in the original image. Similarly, this is the case for $\hat{\mathbf{B}}_{2}, \hat{\mathbf{B}}_{3}$ and $\hat{\mathbf{B}}_{4}$. Note the process of downsampling and upsampling preserves all the low-frequency DCT coefficients while truncating the high-frequency coefficients to zero for each $8 \times 8$ DCT block in the original image.

Since $\hat{\mathbf{B}}$ and $\hat{\mathbf{B}}_{1}$, etc. are related because of Equation (3.5), using DCT transformation matrices $T_{L} T_{2}^{t}$ and $T_{R} T_{2}^{t}$, they derived inverse of $\hat{\mathbf{B}}_{1}$, etc. as follows:

$$
\begin{aligned}
& \hat{\mathbf{B}}_{1}=\left(T_{L} T_{2}^{t}\right)^{t} \hat{\mathbf{B}}\left(T_{L} T_{2}^{t}\right) \\
& \hat{\mathbf{B}}_{2}=\left(T_{L} T_{2}^{t}\right)^{t} \hat{\mathbf{B}}\left(T_{R} T_{2}^{t}\right) \\
& \hat{\mathbf{B}}_{2}=\left(T_{R} T_{2}^{t}\right)^{t} \hat{\mathbf{B}}\left(T_{L} T_{2}^{t}\right) \\
& \hat{\mathbf{B}}_{2}=\left(T_{R} T_{2}^{t}\right)^{t} \hat{\mathbf{B}}\left(T_{R} T_{2}^{t}\right)
\end{aligned}
$$

\subsubsection{Image Doubling through Decomposition followed by Approximation (IDDA)}

Jayanta Mukherjee and Sanjit K. Mitra [13] proposed an image doubling algorithm that first decompose an $8 \times 8$ block of DCT coefficients to four $4 \times 4$ blocks of DCT coefficients. The conversion matrix $\mathbf{A}_{(2,4)}$ in the decomposition step is the same employed in the IHAC algorithm. Then each of these each of these $4 \times 4$ blocks are transform into $8 \times 8$ block by using subband approximation with zero padding. This doubling algorithm follows a similar approach to IDUSZP but with computation is perform differently. Algorithm 3 outlines this image doubling method. 
Algorithm 3 IDDA [13]

Input: $8 \times 8$ block based DCT encoded image.

Output: Upsampled image in the compressed domain.

for each $8 \times 8$ block $\mathbf{B}$ do the following: do

(a) Convert the block to four $4 \times 4$ DCT blocks as follows.

$$
\left[\begin{array}{ll}
\mathbf{B}_{00}^{(4 \times 4)} & \mathbf{B}_{01}^{(4 \times 4)} \\
\mathbf{B}_{10}^{(4 \times 4)} & \mathbf{B}_{11}^{(4 \times 4)}
\end{array}\right]=\mathbf{A}_{(2,4)}^{-1} \mathbf{B} \mathbf{A}_{(2,4)}^{-1^{T}}
$$

(b) Compute the approximate $8 \times 8$-point DCT coefficients from each of $\mathbf{B}_{i j}^{(4 \times 4)}, i=0,1$ and $j=0,1$ from 2.28 or 2.29 . Form four $8 \times 8$ DCT blocks by zero padding each of them (the high frequency components are assigned to zero).

\subsubsection{Image Doubling through Approximation followed by Decomposition (IDAD)}

Jayanta Mukherjee and Sanjit K. Mitra [13] developed a second approach for image doubling. In this approach, the algorithm first transforms an $8 \times 8$ block of DCT coefficients into a $16 \times 16$ block using subband approximation with zero padding. Then the transformed block is decomposed into four $8 \times 8$ blocks of DCT coefficients. This algorithm produces higher image quality than IDDA but with higher computational cost. See algorithm 4 for a description. 
Algorithm 4 IDAD [13]

Input: $8 \times 8$ block based DCT encoded image.

Output: Upsampled image in the compressed domain.

for each $8 \times 8$ DCT block $\mathbf{B}$ do the following: do

(a) Compute the approximate $16 \times 16$-point DCT coefficients from each of $\hat{\mathbf{B}}^{(16 \times 16)}$ from $\mathbf{B}$ using 2.28 or 2.29 by zero padding.

(b) Convert $16 \times 16$ DCT blocks as follows.

$$
\left[\begin{array}{ll}
\mathbf{B}_{00}^{(8 \times 8)} & \mathbf{B}_{01}^{(8 \times 8)} \\
\mathbf{B}_{10}^{(8 \times 8)} & \mathbf{B}_{11}^{(8 \times 8)}
\end{array}\right]=\mathbf{A}_{(2,8)}^{-1} \hat{\mathbf{B}}^{(16 \times 16)} \mathbf{A}_{(2,8)}^{-1^{T}}
$$

end for

\subsection{Resizing with Integral Factors}

Algorithms for image halving and doubling can be extended for downsampling or upsampling an image by integral factors. This means an $L \times M$ downsampling operation reduces the height $(h)$ and width $(w)$ of an image to $\frac{h}{L}$ and $\frac{w}{M}$. Similarly for upsampling operations, the image size increases by $h L \times w M$.

When downsampling an image by a factor of $L \times M$, where $L$ and $M$ are positive integers, one approach is to view this operation as taking $L \times M$ number of $N \times N$ DCT blocks and converting them to a block of $L N \times M N$-DCT. Therefore, when applying subband approximation one must extend Equation (2.29) as follows:

Let $C_{L L}(k, l), 0 \leq k \leq N-1,0 \leq l \leq N-1$ be the DCT coefficients of a $N \times N$ block of the downsampled image. Then the DCT coefficients of an $L N \times M N$ block are approximated by the following equation:

$$
C(k, l)= \begin{cases}\sqrt{L M} C_{L L}(k, l) & : \quad k, l \in\{0,1, \ldots, N-1\} \\ 0 & : \text { otherwise. }\end{cases}
$$

The following subsections outlines one algorithm for downsize images by $L \times M$ factor and one for upsizing images by $L \times M$. Later, we will see how these two algorithms can be used to resize images by arbitrary factors. 


\subsection{1 $L \times M$ Downsampling Algorithm (LMDS)}

The LMDS algorithm follows a similar approach to IHCA algorithm, where a block composition is applied followed by a sub-band approximation. Even though IHCA is more computationally expensive, it is an easier algorithm to adapt for resizing images using integral factors. Algorithm 5 outlines this approach where an $8 \times 8$-DCT block based encode image is reduced by a factor of $L \times M$.

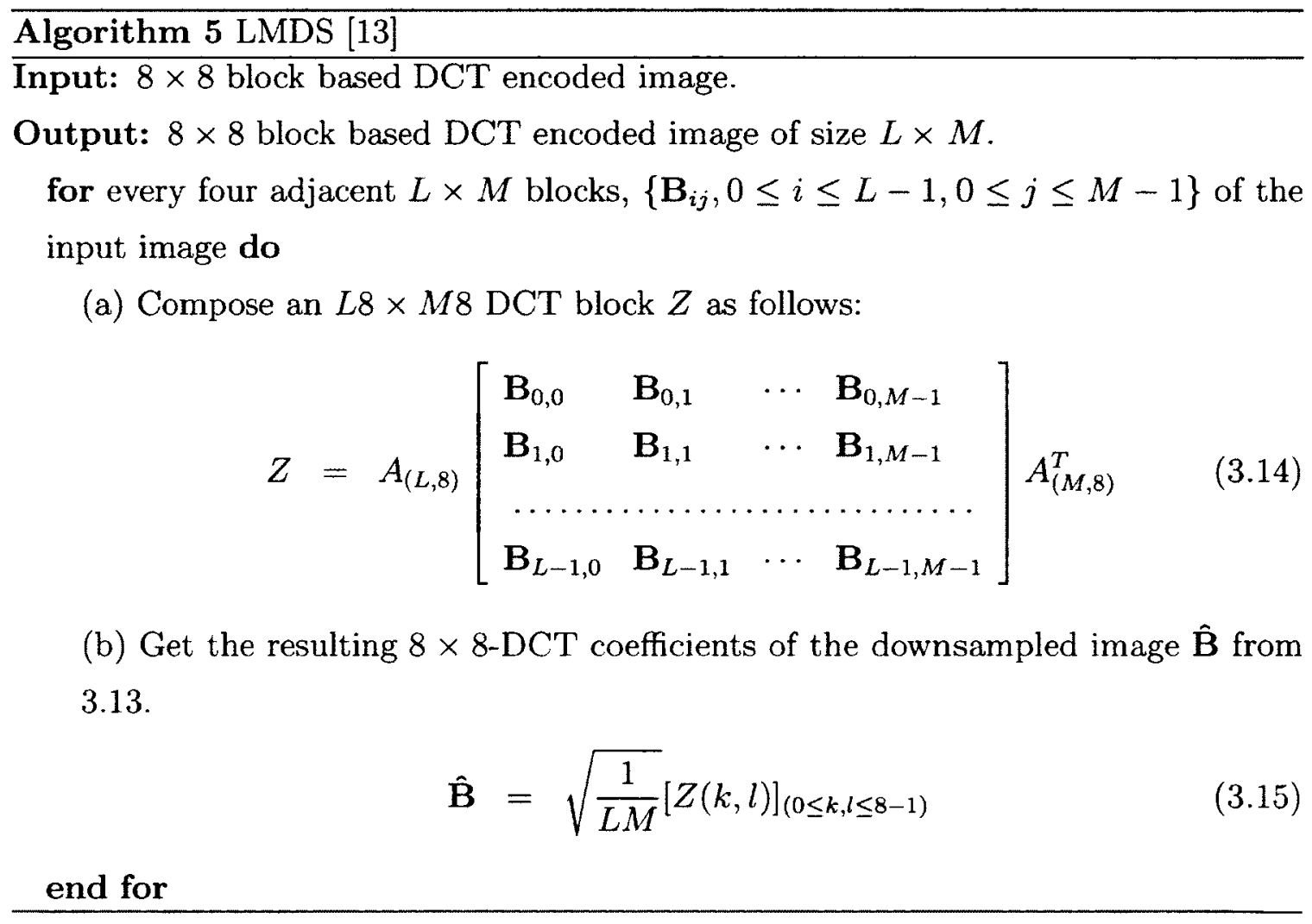

\subsection{2 $L \times M$ Upsampling Algorithm (LMUS)}

Using Equation (3.13) one can convert an image containing $N \times N$ block of DCT coefficients to an $L N \times M N$ block of the upsampled image. After this conversion, apply DCT block decomposition to obtain $L \times M$ numbers of $N \times N$-DCT blocks of the upsampled image. This approach is described in Algorithm 6 where $N=8$. 
Algorithm 6 LMUS [13]

Input: $8 \times 8$ block based DCT encoded image.

Output: $L \times M$ up-sampled image in the compressed domain.

for each $8 \times 8$ block $\mathbf{B}$ do the following: do

(a) Approximate $\mathrm{B}$ to a $L 8 \times M 8$ DCT block as follows:

$$
\hat{\mathbf{B}}^{(L 8 \times M 8)}=\left[\begin{array}{cc}
\sqrt{L M} \mathbf{B} & \mathbf{Z}_{(8,(M-1) 8)} \\
\mathbf{Z}_{((L-1) 8,8)} & \mathbf{Z}_{((L-1) 8,(M-1) 8)}
\end{array}\right]
$$

where $\mathbf{Z}_{(a, b)}$ denotes a matrix of $a \times b$ zero elements.

(b) Decompose $\hat{\mathbf{B}}^{(L 8 \times M 8)}$ into $L \times M$ number of $8 \times 8$ DCT blocks as follows.

$$
\left[\begin{array}{cccc}
\mathbf{B}_{0,0}^{(8 \times 8)} & \mathbf{B}_{0,1}^{(8 \times 8)} & \ldots & \mathbf{B}_{0, M-1}^{(8 \times 8)} \\
\mathbf{B}_{1,0}^{(8 \times 8)} & \mathbf{B}_{1,1}^{(8 \times 8)} & \ldots & \mathbf{B}_{1, M-1}^{(8 \times 8)} \\
\ldots \ldots \ldots & \ldots \ldots \ldots \ldots & \ldots \ldots \ldots \ldots \\
\mathbf{B}_{L-1,0}^{(8 \times 8)} & \mathbf{B}_{L-1,1}^{(8 \times 8)} & \cdots & \mathbf{B}_{L-1, M-1}^{(8 \times 8)}
\end{array}\right]=\mathbf{A}_{(L, 8)}^{-1} \hat{\mathbf{B}}^{(L 8 \times M 8)} \mathbf{A}_{(M, 8)}^{-1^{T}}
$$

end for

\subsection{Resizing with Arbitrary Rational Factors}

Jayanta Mukherjee and Sanjit K. Mitra [13] achieved arbitrary resizing by combining image resizing algorithms that upsample and downsample by integral factors. Arbitrary rational factors are expressed in the form of $\frac{P}{Q} \times \frac{R}{S}$, where $P, Q, R$ and $S$ are positive integers. For example, one approach to resize images by arbitrary factors is first upsample an image by integral factor $P \times R$ followed by downsampling operation by integral factor of $Q \times S$. In the following subsection, we will look at algorithms developed by Mukherjee and Mitra [13] that uses LMDS and LMUS in tandem to resize images by arbitrary factors. 


\subsubsection{UD-Resizing and DU-Resizing Algorithm}

Mukherjee and Mitra [13] developed algorithms to resize by arbitrary factors by employing the LMDS and the LMUS algorithms in tandem. In this case, the resizing factors occured in different order. If the LMUS is followed by LMDS, the image is upsampled first by factor of $P \times R$ and then downsampled by a factor of $Q \times S$. Mukhopadhyay [14] referred this approach as UD-Resizing Algorithm (UDRA). Employing the LMDS and LMUS in the reverse order of UDRA, where downsampling using LMDS is first applied to image followed by upsampling using LMUS algorithm. Mukhopadhyay [14] referred this approach as DU-Resizing Algorithm (DURA). Mukherjee and Mitra [21] provided an example of resizing an HDTV frame of size $1020 \times 1920$ to a NTSC one of size $480 \times 640$. They set $P=8, R=1, Q=17$, and $S=3$ to carry out the resizing. They [21] reported that the UDRA approach produced superior image quality but at higher computational cost than DURA approach. The cost of applying UDRA is $351 M+49 A$ operations per pixel compare to $6.88 M+0.96 A$ operations per pixel of the original image if one applies DURA.

\subsection{Hybrid Resizing Algorithm}

DCT is a separable transform; therefore, DCT resizing can be applied both horizontally and vertically to the coefficients of an image. By using different scale factors for each dimension $x$ and $y$, the image's aspect ratio will be altered. Salazar and Tran [23] for simplicity let $P_{x}=P_{y}$ and $Q_{x}=Q_{y}$, which preserves the image's aspect ratio.

Mukhopandhyay [14] proposed a further generalization and lets $P_{x} \neq P_{x}$ and $Q_{x} \neq Q_{y}$, which alters an image's aspect ratio.

Let $\mathbf{X}$ be a block of DCT coefficients of size $Q N \times S N$. The subband approximated DCT block $\mathrm{Y}$ of size $P N \times R N$ is given by the following expression.

$$
\mathbf{Y}=\sqrt{\frac{P R}{Q S}} \hat{\mathbf{X}}
$$

where $\hat{\mathbf{X}}$ are derived by truncating or zero-padding $\mathbf{X}$ coefficients in the respective dimensions depending upon the ordered relationship between $P$ and $Q$, and $R$ and $S$.

Using the above Equation 3.17 the HRA approach inputs $Q \times S$ adjacent DCT 
blocks can converts them to $P \times R$ DCT blocks. The algorithm for this computation is describe in Algorithm 7.

Algorithm 7 Hybrid_Resizing_Algorithm (HRA) [14]

Input: $Q \times S$ adjacent DCT blocks, each of size $N \times N$.

Output: $P \times R$ adjacent DCT blocks, each of size $N \times N$.

1. Block composition: In this step, $Q \times S$ input blocks are composed into a block of size $Q N \times S N$.

2. Subband approximation: Let $\mathrm{Y}$ denote the $P N \times R N$ subband approximated block obtained from the input block $\mathbf{X}$ of size $Q N \times S N$. Then the relationship between $\mathbf{Y}$ and $\mathbf{X}$ is expressed in the following equation:

$$
\begin{aligned}
& \mathbf{Y}=\sqrt{\frac{P R}{Q S}}[\mathbf{X}]_{0 \leq i \leq P N, 0 \leq j \leq R N} \quad: \quad P \leq Q, R \leq S, \\
& =\sqrt{\frac{P R}{Q S}}\left[\begin{array}{c}
{[\mathbf{X}]_{0 \leq i<Q N, 0 \leq j<R N}^{-}} \\
0_{(P-Q) N \times R N}
\end{array}\right]: P>Q, R \leq S, \\
& =\sqrt{\frac{P R}{Q S}}\left[[\mathbf{X}]_{0 \leq i<P N, 0 \leq j<S N} \quad 0_{P N \times(R-S) N}\right]: P \leq Q, R>S,>S \text {, }
\end{aligned}
$$

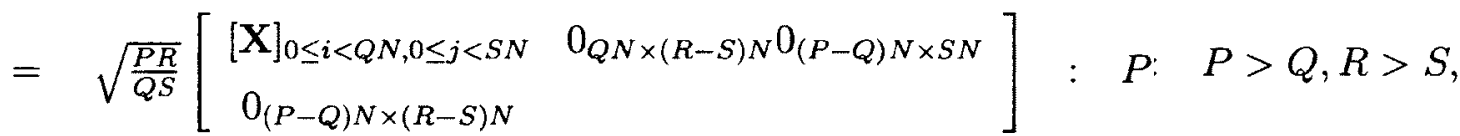

3. Block decomposition: In this step, $\mathrm{Y}$ is decomposed into $P \times R$ blocks, each of size $N \times N$

Mukhopandhyay [14] reported that HRA generated images with similar quality as UDRA approach. In his paper, he resized an HDTV frame to NTSC frame resolution, where he reported that HRA takes 42.5 multiplications and 75.15 additions for processing a pixel of the original image. This has a lower computational cost than UDRA which takes $351 M+49 A$ operations per pixel but not as low as DURA which takes $6.88 M+0.96 A$. Even though DURA has the lowest computational cost, Mukhopandhyay observed images resized using DURA technique suffers heavily from the appearance of blocking artifacts. 


\subsection{Summary}

In this chapter, thirteen image resizing algorithms that work in the compressed domain were presented. The chapter first outlined several algorithms for image halving and doubling, which eventually lead to research on resizing images by integral and arbitrary factors. All the researchers developing these algorithms assumed that image were compressed using a popular $8 \times 8$ DCT block framework and that inverse quantization was applied on the DCT coefficients. In the next chapter, the main contribution of the thesis is outlined that adapts these algorithms to work on H.264/AVC frames. 


\section{Chapter 4}

\section{DCT Domain H.264/AVC Frame Resizing Algorithms}

\subsection{Introduction}

This chapter presents how algorithms outlined in Chapter 3 were adapted to resize H.264/AVC frames in the frequency domain. The chapter will start with a section on H.264/AVC halving algorithms followed by its opposite operation on doubling algorithms. A section on resizing using integral factors will be covered, which will lead to algorithms resizing H.264/AVC frames by arbitrary factors.

\subsection{Halving H.264/AVC Frames}

This section covers algorithms to halve a H.264/AVC frame. Halving algorithms are algorithms that convert a H.264/AVC frame of size $N \times N$ to size of $N / 2 \times N / 2$.

\subsubsection{Image Halving through Bilinear Interpolation (IHBI)}

The bilinear approach was adapted to downsize frames containing blocks of $4 \times 4$ DCT coefficients. As previously presented, H.264/AVC employs $4 \times 4$ integer transform, an approximate form of the DCT, to form blocks of $4 \times 4$ DCT coefficients. Also, during forward transformation, quantization and scaling is applied together with the core transform. The downsampling $4 \times 4$ filter matrices $h_{i}$ and $g_{i}$ was created as follows:

$$
\begin{aligned}
& h_{2}=g_{1}^{t}=g_{3}^{t}=h_{1}=\left[\begin{array}{l}
\mathbf{u}_{2 \times 4} \\
\mathbf{o}_{2 \times 4}
\end{array}\right] \\
& h_{4}=g_{2}^{t}=g_{4}^{t}=h_{3}=\left[\begin{array}{l}
\mathbf{o}_{2 \times 4} \\
\mathbf{u}_{2 \times 4}
\end{array}\right]
\end{aligned}
$$

where $\mathbf{u}_{2 \times 4}$ is defined in Equation (4.2) and $\mathbf{o}_{2 \times 4}$ is a $2 \times 4$ zero matrix.

$$
\mathbf{u}_{2 \times 4}=\left[\begin{array}{cccc}
.5 & .5 & 0 & 0 \\
0 & 0 & .5 & .5
\end{array}\right]
$$


A floating-point forward DCT was applied to the filter matrices $h_{i}$ and $g_{i}$, which generated the following matrices:

$$
\begin{aligned}
& \operatorname{DCT}\left(h_{2}\right)=\operatorname{DCT}\left(g_{1}^{t}\right)=\operatorname{DCT}\left(g_{3}^{t}\right)=\operatorname{DCT}\left(h_{1}\right) \\
& =\left[\begin{array}{cccc}
0.5000 & 0 & 0 & 0 \\
0.4620 & 0.1768 & 0 & -0.0732 \\
0 & 0.4620 & 0 & -0.1914 \\
-0.1914 & 0.4620 & 0 & -0.1768
\end{array}\right] \\
& \operatorname{DCT}\left(h_{4}\right)=\operatorname{DCT}\left(g_{2}^{t}\right)=\operatorname{DCT}\left(g_{4}^{t}\right)=\operatorname{DCT}\left(h_{3}\right) \\
& =\left[\begin{array}{cccc}
0.5000 & 0 & 0 & 0 \\
-0.4620 & 0.1768 & 0 & -0.0732 \\
0 & -0.4620 & 0 & 0.1914 \\
0.1914 & 0.4620 & 0 & -0.1768
\end{array}\right]
\end{aligned}
$$

Then apply the filter matrices as outlined Equation (4.4) on H.264/AVC frame to half the image.

$$
\operatorname{DCT}(c)=T c T^{t}=\sum_{i=1}^{4} \operatorname{DCT}\left(h_{i}\right) \operatorname{DCT}\left(c_{i}\right) \operatorname{DCT}\left(g_{i}\right)
$$

\subsubsection{Image Halving through Low-Pass Filtering followed by Downsampling (IHFDS)}

A similar method based on Dugad and Ahuja [12] image halving algorithm to work on frames containing blocks of $4 \times 4$ DCT coefficients generated from a H.264/AVC forward transform was developed. Instead of the $\mathbf{B}_{i}$ representing blocks of $8 \times 8 \mathrm{DCT}$ coefficients, they are blocks of $4 \times 4$ DCT coefficients. The algorithm can be viewed as image halving through low-pass filtering followed by downsampling operation. We will first cover the downsampling part and then the low-pass filtering operation.

\section{Downsampling H.264/AVC Frames}

The matrix $T_{L}$ and $T_{R}$ are $4 \times 2$ sub-matrices of the $4 \times 4$ forward DCT $T_{f 4}$.

$$
T_{f 4}=\left[\begin{array}{lll}
T_{L} & \mid & T_{R}
\end{array}\right]
$$




$$
=\left[\begin{array}{cc|cc}
0.5 & 0.5 & 0.5 & 0.5 \\
0.6533 & 0.2706 & -0.2706 & -0.6533 \\
0.5 & -0.5 & -0.5 & 0.5 \\
0.2706 & -0.6533 & 0.6533 & -0.2706
\end{array}\right]
$$

The matrix $T_{2}$ is $2 \times 2$ forward DCT.

$$
\begin{aligned}
T_{2} & =T_{2}^{t} \\
& =\left[\begin{array}{cc}
0.7071 & 0.7071 \\
0.7071 & -0.7071
\end{array}\right]
\end{aligned}
$$

Therefore, the product of the matrices $T_{L} T_{2}^{t}$ and $T_{R} T_{2}^{t}$ are:

$$
\begin{aligned}
T_{L} T_{2}^{t}= & {\left[\begin{array}{cc}
0.7071 & 0 \\
0.6533 & 0.2706 \\
0 & 0.7071 \\
-0.2706 & 0.6533
\end{array}\right] } \\
T_{R} T_{2}^{t}= & {\left[\begin{array}{cc}
0.7071 & 0 \\
-0.6533 & 0.2706 \\
0 & -0.7071 \\
0.2706 & 0.6533
\end{array}\right] }
\end{aligned}
$$

\section{Low-Pass Filter Matrix}

The low-pass filter represented by the matrix $M$ to downsize H.264/AVC frames is different than the $8 \times 8$ DCT version. Initially, $2 \times 2$ identity matrix was employed for the matrix $M$ but this produced poor image quality. The low-pass filter matrix is actually a form of subband approximation as outlined in Equation (2.29). Therefore, filter matrix became:

$$
M \stackrel{\text { def }}{=}\left[\begin{array}{cc}
1 / \sqrt{2} & 0 \\
0 & 1 / \sqrt{2} \\
0 & 0 \\
0 & 0
\end{array}\right]
$$


Then $M$ was multiply with the downsampling matrices $T_{L} T_{2}^{t}$ and $T_{R} T_{2}^{t}$ outlined in Equation (4.7) produced the following matrices:

$$
\begin{aligned}
T_{L} T_{2}^{t} M^{t} & =\left[\begin{array}{cccc}
0.5 & 0 & 0 & 0 \\
0.46194 & 0.191342 & 0 & 0 \\
0 & 0.5 & 0 & 0 \\
-0.191342 & 0.46194 & 0 & 0
\end{array}\right] \\
T_{R} T_{2}^{t} M^{t}= & {\left[\begin{array}{cccc}
0.5 & 0 & 0 & 0 \\
-0.46194 & 0.191342 & 0 & 0 \\
0 & -0.5 & 0 & 0 \\
0.191342 & 0.46194 & 0 & 0
\end{array}\right] }
\end{aligned}
$$

Let $\mathbf{B}_{1}, \mathbf{B}_{2}, \mathbf{B}_{3}$ and $\mathbf{B}_{4}$ denote four adjacent $4 \times 4$ blocks of DCT coefficients that form $2 \times 2$ block. Therefore, the downsampled image of $\hat{\mathbf{B}}$ is derived using the following equation:

$$
\begin{aligned}
\hat{\mathbf{B}}= & \left(T_{L} T_{4}^{t} M^{t}\right) \mathbf{B}_{1}\left(T_{L} T_{4}^{t} M^{t}\right)^{t}+\left(T_{L} T_{4}^{t} M^{t}\right) \mathbf{B}_{2}\left(T_{R} T_{4}^{t} M^{t}\right)^{t} \\
& +\left(T_{R} T_{4}^{t} M^{t}\right) \mathbf{B}_{3}\left(T_{L} T_{4}^{t} M^{t}\right)^{t}+\left(T_{R} T_{4}^{t} M^{t}\right) \mathbf{B}_{4}\left(T_{R} T_{4}^{t} M^{t}\right)^{t}
\end{aligned}
$$

\subsubsection{Image Halving through Approximation followed by Composition (IHAC)}

This algorithm is derived on method developed by Mukherjee and Mitra[13]. For the subband approximation stage, the low-pass truncated approximation 2.29 was used. Algorithm 8 describes this implementation. 
Algorithm 8 IHAC for H.264/AVC Frames

Input: $4 \times 4$ block H.264 based DCT encoded image.

Output: $4 \times 4$ block based DCT encoded downsampled image.

for every four adjacent $4 \times 4, \mathbf{B}_{00}, \mathbf{B}_{01}, \mathbf{B}_{10}, \mathbf{B}_{11}$ of the input image do

1. Subband approximation: Get corresponding $2 \times 2$ 2-point DCT blocks $\left\{\hat{\mathbf{B}}_{i j}^{(2 \times 2)}\right\}, i=0,1$ and $j=0,1$ using 2.29 , low-pass truncated approximation, as follows.

$$
\hat{\mathbf{B}}_{i j}^{(2 \times 2)}=\frac{1}{2}\left[\mathbf{B}_{i j}(k, l)\right]_{0 \leq k, l \leq 1}
$$

2. Block composition: Convert four $2 \times 2$ DCT blocks to a $4 \times 4$ DCT block $\mathrm{B}_{d}$ as follows.

$$
\mathbf{B}_{d}=\mathbf{A}_{(2,2)}\left[\begin{array}{cc}
\hat{\mathbf{B}}_{00}^{(2 \times 2)} & \hat{\mathbf{B}}_{01}^{(2 \times 2)} \\
\hat{\mathbf{B}}_{10}^{(2 \times 2)} & \hat{\mathbf{B}}_{11}^{(2 \times 2)}
\end{array}\right] \mathbf{A}_{(2,2)}^{T}
$$

end for

The block composition matrix $\mathbf{A}_{(2,2)}$ is expressed as follows:

$$
\mathbf{A}_{(2,2)}=\left[\begin{array}{cccc}
1 / \sqrt{2} & 0 & 1 / \sqrt{2} & 0 \\
0.6533 & 0.2706 & -0.6533 & 0.2706 \\
0 & 1 / \sqrt{2} & 0 & -1 / \sqrt{2} \\
-0.2706 & 0.6533 & 0.2706 & 0.6533
\end{array}\right]
$$

\subsubsection{Image Halving through Composition followed by Approximation (IHCA)}

This is an adaption of image halving algorithm developed by Mukherjee and Mitra [13]. This implementation used the low-pass truncated approximation 2.29 for the subband approximation. Algorithm 9 outlines this implementation. 
Algorithm 9 IHCA for H.264/AVC Frames

Input: $4 \times 4$ block H.264 based DCT encoded image.

Output: $4 \times 4$ block based DCT encoded downsampled image.

for every four adjacent $4 \times 4, \mathbf{B}_{00}, \mathbf{B}_{01}, \mathbf{B}_{10}, \mathbf{B}_{11}$ of the input image do

1. Block composition: Convert four adjacent $4 \times 4$ DCT blocks to a $8 \times 8$ DCT block $\mathbf{B}^{(8 \times 8)}$ as follows.

$$
\mathbf{B}^{(8 \times 8)}=\mathbf{A}_{(2,4)}\left[\begin{array}{ll}
\mathbf{B}_{00} & \mathbf{B}_{01} \\
\mathbf{B}_{10} & \mathbf{B}_{11}
\end{array}\right] \mathbf{A}_{(2,4)}^{T}
$$

2. Subband approximation: Get the resulting $4 \times 4$ block $\mathbf{B}^{(4 \times 4)}$ using 2.29 , low-pass truncated approximation, as follows.

$$
\mathbf{B}^{(4 \times 4)}=\frac{1}{2}\left[\mathbf{B}^{(8 \times 8)}(k, l)\right] 0 \leq k, l \leq 4
$$

end for

The block composition matrix $\mathbf{A}_{(2,4)}$ is outlined as follows:

$$
\mathbf{A}_{(2,4)}=\left[\begin{array}{cccccccc}
1 / \sqrt{2} & 0 & 0 & 0 & 1 / \sqrt{2} & 0 & 0 & 0 \\
0.6407 & 0.2940 & -0.0528 & 0.0162 & -0.6407 & 0.2940 & 0.0528 & 0.0162 \\
0 & 1 / \sqrt{2} & 0 & 0 & 0 & -1 / \sqrt{2} & 0 & 0 \\
-0.2250 & 0.5594 & 0.3629 & -0.0690 & 0.2250 & 0.5594 & -0.3629 & -0.0690 \\
0 & 0 & 1 / \sqrt{2} & 0 & 0 & 0 & 1 / \sqrt{2} & 0 \\
0.1503 & -0.2492 & 0.5432 & 0.3468 & -0.1503 & -0.2492 & -0.5432 & 0.3468 \\
0 & 0 & 0 & 1 / \sqrt{2} & 0 & 0 & 0 & -1 / \sqrt{2} \\
-0.1274 & 0.1964 & -0.2654 & 0.6122 & 0.1274 & 0.1964 & 0.2654 & 0.6122
\end{array}\right]
$$

\subsection{Doubling H.264/AVC Frames}

This section covers algorithms to double an H.264/AVC frame. Image doubling algorithms are algorithms that convert a H.264/AVC frame of size $N \times N$ to size of $2 N \times 2 N$. 


\subsubsection{Image Doubling through Zero-Order Interpolation (IDZOI)}

For upsampling H.264/AVC frames using zero-order interpoloation, a similar approach outlined in the image halving version of bilinear interpolation was followed. The basic idea of this method is to read each $4 \times 4$ block of DCT coefficients $\hat{\mathbf{B}}$ from a downsampled image and create four new $4 \times 4$ blocks of DCT coefficients, $\hat{\mathbf{B}}_{1}, \hat{\mathbf{B}}_{2}, \hat{\mathbf{B}}_{3}$ and $\hat{\mathbf{B}}_{4}$ for the upsampled image (See Figure 4.1 ).

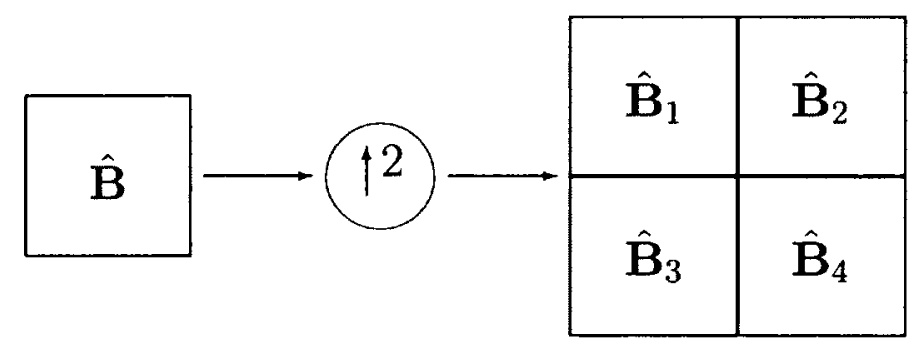

Figure 4.1: Image doubling through zero-order interpolation.

Let $\hat{\mathbf{b}}=\operatorname{IDCT}(\hat{\mathbf{B}})$, which $\hat{\mathbf{b}}$ is the spatial representation of $\hat{\mathbf{B}}$. In the spatial domain, a simple and straight forward approach to derive the upsample blocks $\hat{\mathbf{b}}_{1}, \hat{\mathbf{b}}_{2}, \hat{\mathbf{b}}_{3}$ and $\hat{b}_{4}$ can be represented using matrix notation as follows:

$$
\begin{aligned}
& \hat{\mathbf{b}}_{1}=g_{1} \hat{\mathbf{b}} h_{1} \\
& \hat{\mathbf{b}}_{2}=g_{1} \hat{\mathbf{b}} h_{2} \\
& \hat{\mathbf{b}}_{3}=g_{2} \hat{\mathbf{b}} h_{1} \\
& \hat{\mathbf{b}}_{4}=g_{2} \hat{\mathbf{b}} h_{2}
\end{aligned}
$$

where the upsampling filters, $h_{1}, h_{2}, g_{1}$ and $g_{2}$ are given by

$$
\begin{aligned}
& h_{1}=g_{1}^{t}=\left[\begin{array}{l}
\mathbf{u}_{2 \times 4} \\
\mathbf{o}_{2 \times 4}
\end{array}\right] \\
& h_{2}=g_{2}^{t}=\left[\begin{array}{l}
\mathbf{o}_{2 \times 4} \\
\mathbf{u}_{2 \times 4}
\end{array}\right]
\end{aligned}
$$

where $u_{2 \times 4}$ is defined in Equation (4.14) and $o_{2 \times 4}$ zero matrix. 


$$
\mathbf{u}_{2 \times 4}=\left[\begin{array}{cccc}
1 & 1 & 0 & 0 \\
0 & 0 & 1 & 1
\end{array}\right]
$$

In the compressed domain, the straight forward approach expressed in Equation (4.12) becomes:

$$
\begin{aligned}
& \operatorname{DCT}\left(\hat{\mathbf{b}}_{1}\right)=\operatorname{DCT}\left(g_{1}\right) \operatorname{DCT}(\hat{\mathbf{b}}) \operatorname{DCT}\left(h_{1}\right) \\
& \operatorname{DCT}\left(\hat{\mathbf{b}}_{2}\right)=\operatorname{DCT}\left(g_{1}\right) \operatorname{DCT}(\hat{\mathbf{b}}) \operatorname{DCT}\left(h_{2}\right) \\
& \operatorname{DCT}\left(\hat{\mathbf{b}}_{3}\right)=\operatorname{DCT}\left(g_{2}\right) \operatorname{DCT}(\hat{\mathbf{b}}) \operatorname{DCT}\left(h_{1}\right) \\
& \operatorname{DCT}\left(\hat{\mathbf{b}}_{4}\right)=\operatorname{DCT}\left(g_{2}\right) \operatorname{DCT}(\hat{\mathbf{b}}) \operatorname{DCT}\left(h_{2}\right)
\end{aligned}
$$

where DCT is a floating-point forward DCT. A rounding operation was applied to convert each $\operatorname{DCT}\left(\hat{\mathbf{b}}_{i}\right)$ into a $\operatorname{DCT}\left(\hat{\mathbf{b}}_{i}\right)=\hat{\mathbf{B}}_{i}$.

Even though the upsampling matrix $h_{1}, h_{2}, g_{1}$ and $g_{2}$ are sparse, the forward DCT versions of them are not, which are displayed Equation (4.16).

$$
\begin{aligned}
\operatorname{DCT}\left(h_{1}\right) & =\operatorname{DCT}\left(g_{1}\right)^{t} \\
& =\left[\begin{array}{cccc}
1 & 0 & 0 & 0 \\
0.9239 & 0.3536 & 0 & -0.1465 \\
0 & 0.9239 & 0 & 0.3827 \\
0.3827 & 0.8536 & 0 & -0.3536
\end{array}\right] \\
\operatorname{DCT}\left(h_{2}\right) & =\operatorname{DCT}\left(g_{2}\right)^{t} \\
& =\left[\begin{array}{cccc}
1 & 0 & 0 & 0 \\
-0.9239 & 0.3536 & 0 & -0.1465 \\
0 & -0.9239 & 0 & 0.3827 \\
0.3827 & 0.8536 & 0 & -0.3536
\end{array}\right]
\end{aligned}
$$

This lack of sparseness contributes to higher computational cost. Subsequent section on Computational Cost will provide more detail explanation.

\subsubsection{Image Doubling through Upsampling followed by Zero-Padding (IDUSZP)}

This upsampling approach is the inverse to the IHFDS [12]. The technique first performs decomposition (upsampling) before the subband approximation (filtering) 
is applied. This implementation is based on algorithm developed by Dugad and Ahuja [12] and is adapted to upsample H.264 frames as follows.

For each $4 \times 4 \hat{\mathbf{B}}$ in the downsampled image, extract $\hat{\mathbf{b}}=\operatorname{IDCT}(\hat{\mathbf{B}})$. Partition $\hat{\mathbf{b}}$ into $2 \times 2$ sub-blocks so that

$$
\hat{\mathbf{b}}=\left[\begin{array}{ll}
\hat{\mathbf{b}}_{1} & \hat{\mathbf{b}}_{2} \\
\hat{\mathbf{b}}_{3} & \hat{\mathbf{b}}_{4}
\end{array}\right]
$$

Then compute $\hat{\mathbf{B}}_{1}=\operatorname{DCT}\left(\hat{\mathbf{b}}_{1}\right)$ and etc. Since $\hat{\mathbf{B}}_{1}$ contains all the low-frequency DCT coefficients of the corresponding $4 \times 4$ original image, create the following matrix

$$
\hat{\mathbf{B}}_{1}=\left[\begin{array}{ll}
\hat{\mathbf{B}} & \mathbf{O} \\
\mathbf{O} & \mathbf{O}
\end{array}\right]
$$

where $\mathrm{O}$ denotes a $2 \times 2$ zero matrix.

Therefore, $\hat{\mathbf{B}}_{1}$ DCT block corresponds to $\mathbf{B}_{1}$ DCT block in the original image. Similarly, this is the case for $\hat{\mathbf{B}}_{2}, \hat{\mathbf{B}}_{3}$ and $\hat{\mathbf{B}}_{4}$. Note the process of downsampling and upsampling preserves all the low-frequency DCT coefficients while truncating the high-frequency coefficients to zero for each $4 \times 4$ DCT block in the original image.

Since $\hat{\mathbf{B}}$ and $\hat{\mathbf{B}}_{1}$, etc. are related because of Equation (3.5), the DCT transformation matrices $T_{L} T_{2}^{t}$ and $T_{R} T_{2}^{t}$ denoted in Equation (4.7) we derived the inverse of $\hat{\mathbf{B}}_{1}$, $\hat{\mathbf{B}}_{2}, \hat{\mathbf{B}}_{3}$, and $\hat{\mathbf{B}}_{4}$ as follows:

$$
\begin{aligned}
& \hat{\mathbf{B}}_{1}=\left(T_{L} T_{2}^{t}\right)^{t} \hat{\mathbf{B}}\left(T_{L} T_{2}^{t}\right) \\
& \hat{\mathbf{B}}_{2}=\left(T_{L} T_{2}^{t}\right)^{t} \hat{\mathbf{B}}\left(T_{R} T_{2}^{t}\right) \\
& \hat{\mathbf{B}}_{3}=\left(T_{R} T_{2}^{t}\right)^{t} \hat{\mathbf{B}}\left(T_{L} T_{2}^{t}\right) \\
& \hat{\mathbf{B}}_{4}=\left(T_{R} T_{2}^{t}\right)^{t} \hat{\mathbf{B}}\left(T_{R} T_{2}^{t}\right)
\end{aligned}
$$

As in the case for the downsampling method, the $4 \times 2$ low-pass filter for upsizing can be defined independently to the DCT transforms, which is as follows:

$$
M \stackrel{\text { def }}{=}\left[\begin{array}{cc}
\sqrt{2} & 0 \\
0 & \sqrt{2} \\
0 & 0 \\
0 & 0
\end{array}\right]
$$

The non-zero elements of $M$ were derived from subband approximation using eq. (2.29), low-pass truncated approximation. Then $M$ is multiple with the transform 
matrices $T_{L} T_{2}^{t}$ and $T_{R} T_{2}^{t}$ produced the following matrices:

$$
\begin{aligned}
T_{L} T_{2}^{t} M^{t}= & {\left[\begin{array}{cccc}
1.0 & 0 & 0 & 0 \\
0.9239 & 0.3827 & 0 & 0 \\
0 & 1.0 & 0 & 0 \\
-0.3827 & 0.9239 & 0 & 0
\end{array}\right] } \\
T_{R} T_{2}^{t} M^{t}= & {\left[\begin{array}{cccc}
1.0 & 0 & 0 & 0 \\
-0.9239 & 0.3827 & 0 & 0 \\
0 & -1.0 & 0 & 0 \\
0.3827 & 0.9239 & 0 & 0
\end{array}\right] }
\end{aligned}
$$

Then apply the following equation on $\hat{\mathbf{B}}$ to create four matrices $\hat{\mathbf{B}}_{1}, \hat{\mathbf{B}}_{2}, \hat{\mathbf{B}}_{3}$ and $\hat{\mathbf{B}}_{4}$.

$$
\begin{aligned}
& \hat{\mathbf{B}}_{1}=\left(T_{L} T_{2}^{t} M^{t}\right) \hat{\mathbf{B}}\left(T_{L} T_{2}^{t} M^{t}\right) \\
& \hat{\mathbf{B}}_{2}=\left(T_{L} T_{2}^{t} M^{t}\right) \hat{\mathbf{B}}\left(T_{R} T_{2}^{t} M^{t}\right) \\
& \hat{\mathbf{B}}_{3}=\left(T_{R} T_{2}^{t} M^{t}\right) \hat{\mathbf{B}}\left(T_{L} T_{2}^{t} M^{t}\right) \\
& \hat{\mathbf{B}}_{4}=\left(T_{R} T_{2}^{t} M^{t}\right) \hat{\mathbf{B}}\left(T_{R} T_{2}^{t} M^{t}\right)
\end{aligned}
$$

Using these four matrices we form a $2 \times 2$ block where each block contains $4 \times 4$ blocks of DCT coefficients. In summary, we started with input $4 \times 4$ block of DCT coefficients $\hat{\mathbf{B}}$ and doubled it size by forming a $2 \times 2$ block containing four adjacent $4 \times 4$ blocks of DCT coefficients created in Equation (4.20).

\subsubsection{Image Doubling through Decomposition followed by Approximation (IDDA)}

This image doubling algorithm was adapted from a method developed by Mukherjee and Mitra [13]. The algorithm first decompose a $4 \times 4$ block of DCT coefficients into four $2 \times 2$ blocks. Then each of these $2 \times 2$ blocks are transformed into $4 \times 4$ blocks of DCT coefficients using subband approximation with zero padding. Algorithm 10 outlines this image doubling method.

We simplified decomposition step. In the original algorithm, Mukherjee and Mitra applied an inverse on conversion matrix which is expressed as follows:

$$
\mathbf{A}_{(2,4)}^{-1} \mathbf{B A}_{(2,4)}^{-1^{T}}
$$


Where $T$ denotes a transpose operation and -1 denotes an inverse operation. We discovered that conversion matrix $\mathbf{A}_{(2,2)}$ and $\mathbf{A}_{(2,4)}$ are orthonormal. In other words $\mathbf{I}=\mathbf{A}_{(2,2)} \mathbf{A}_{(2,2)}^{T}$, where $\mathbf{I}$ is the identity matrix. Therefore, $\mathbf{A}_{(2,2)}^{T}=\mathbf{A}_{(2,2)}^{-1}$.

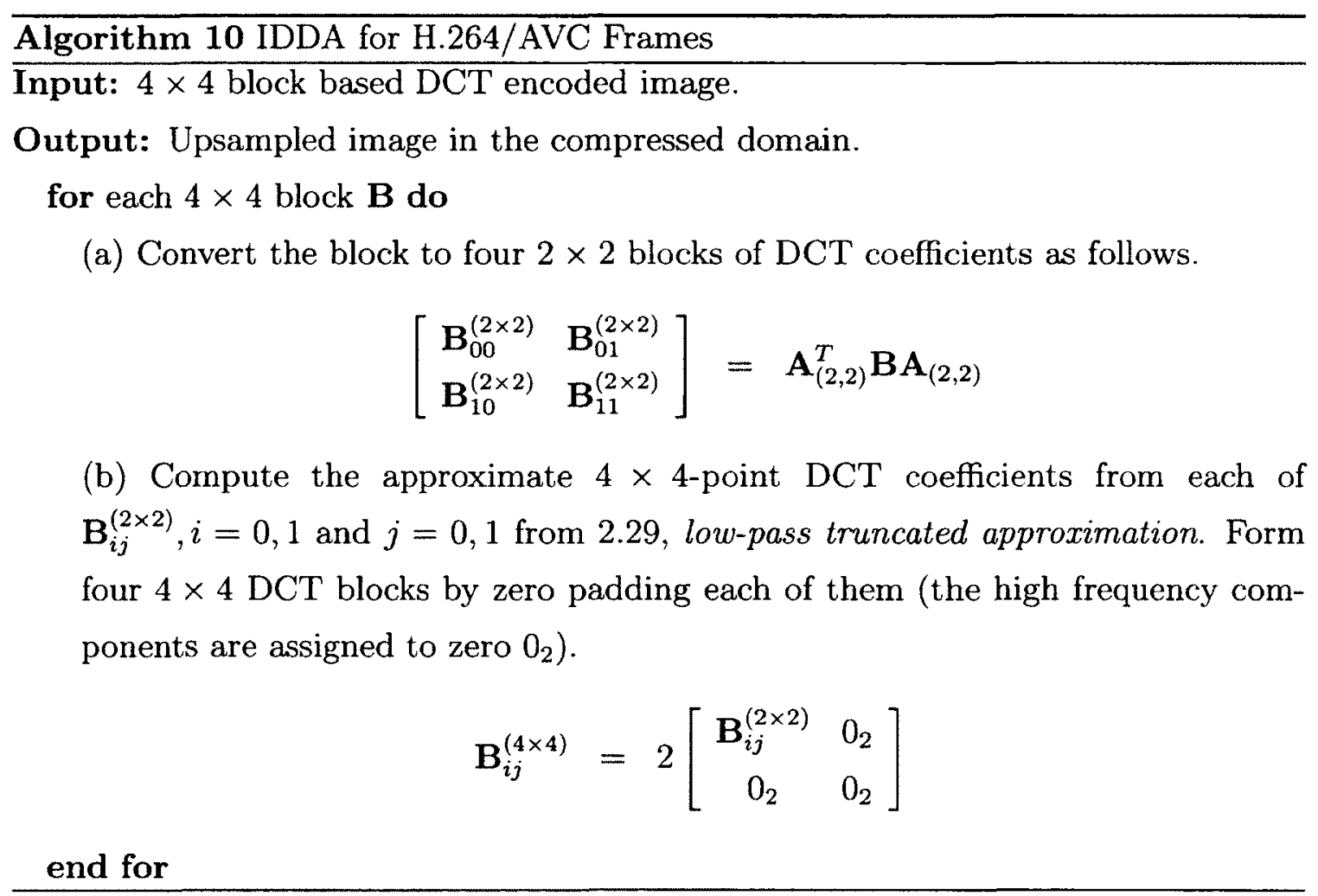

\subsubsection{Image Doubling through Approximation followed by Decomposition (IDAD)}

This image doubling algorithm was adapted from a method developed by Mukherjee and Mitra [13]. The algorithm first transforms an $4 \times 4$ block of DCT coefficients into a $8 \times 8$ block using subband approximation with zero padding. Then the transformed block is decomposed into four $4 \times 4$ blocks of DCT coefficients. The algorithm produced images with higher quality but with a higher computational cost than IDDA. Algorithm 11 outlines a description of the algorithm.

As we did for the IDDA in the decomposition step, we simplified by applying the transpose only on the first composition matrix. 

Algorithm 11 IDAD for H.264/AVC Frames
Input: $4 \times 4$ block based DCT encoded image.

Output: Upsampled image in the compressed domain.

for each $4 \times 4$ block of DCT coefficients B do

(a) Compute the approximate $8 \times 8$-point DCT coefficients from each of $\hat{\mathbf{B}}^{(8 \times 8)}$ from $\mathbf{B}$ using 2.29, low-passed truncated approximation, by zero padding.

$$
\hat{\mathbf{B}}^{(8 \times 8)}=2\left[\begin{array}{cc}
\mathrm{B} & 0_{4} \\
0_{4} & 0_{4}
\end{array}\right]
$$

(b) Convert $8 \times 8 \mathrm{DCT}$ blocks as follows.

$$
\left[\begin{array}{ll}
\mathbf{B}_{00}^{(4 \times 4)} & \mathbf{B}_{01}^{(4 \times 4)} \\
\mathbf{B}_{10}^{(4 \times 4)} & \mathbf{B}_{11}^{(4 \times 4)}
\end{array}\right]=\mathbf{A}_{(2,4)}^{T} \hat{\mathbf{B}}^{(8 \times 8)} \mathbf{A}_{(2,4)}
$$

end for

\subsection{Resizing with Integral Factors}

In this section, algorithms that resize images by integral factors are covered. This means an $L \times M$ downsampling operation reduces the height $(h)$ and width $(w)$ by $\frac{h}{L}$ and $\frac{w}{M}$. Similarly for upsampling operations, the image size increases by $h L \times w M$. The following subsection will outline two algorithms that were adapted to resize H.264/AVC frames by integral factors.

\subsection{1 $L \times M$ Downsampling Algorithm (LMDS)}

This algorithm was first presented by Mukherjee and Mitra [13]. The LMDS algorithm follows a similar approach to IHCA algorithm, where a block composition is applied followed by a subband approximation. Even though IHCA is more computationally expensive, it is an easier algorithm to adapt for resizing images using integral factors. Algorithm 12 outlines an adapt approach where an $4 \times 4$-DCT block based image is reduced by a factor of $L \times M$. 
Algorithm 12 LMDS for H.264/AVC Frames

Input: $4 \times 4$ block based DCT encoded image.

Output: $4 \times 4$ block based DCT encoded image of size $L \times M$.

for every adjacent $L \times M$ blocks, $\left\{\mathbf{B}_{i j}, 0 \leq i \leq L-1,0 \leq j \leq M-1\right\}$ of the input image do

(a) Compose an $L 4 \times M 4$ DCT block $Z$ as follows:

$$
Z=A_{(L, 4)}\left[\begin{array}{cccc}
\mathbf{B}_{0,0} & \mathbf{B}_{0,1} & \cdots & \mathbf{B}_{0, M-1} \\
\mathbf{B}_{1,0} & \mathbf{B}_{1,1} & \cdots & \mathbf{B}_{1, M-1} \\
\ldots \ldots & \ldots \ldots \ldots \ldots \ldots \ldots \ldots \ldots \\
\mathbf{B}_{L-1,0} & \mathbf{B}_{L-1,1} & \cdots & \mathbf{B}_{L-1, M-1}
\end{array}\right] A_{(M, 4)}^{T}
$$

(b) Get the resulting $4 \times 4$-DCT coefficients of the downsampled image $\hat{B}$ from 3.13 .

$$
\hat{\mathbf{B}}=\sqrt{\frac{1}{L M}}[Z(k, l)]_{(0 \leq k, l \leq 4-1)}
$$

end for

\subsection{2 $L \times M$ Upsampling Algorithm (LMUS)}

Mukherjee and Mitra [13] developed a similar algorithm for upsampling an image by an integral factor of $L \times M$. Algorithm 13 outlines an adapt approach to increase the size of an image composed of $4 \times 4$-DCT blocks by a factor of $L \times M$. 
Algorithm 13 LMUS for H.264/AVC Frames

Input: $4 \times 4$ block based DCT encoded image.

Output: $L \times M$ up-sampled image in the compressed domain.

for each $4 \times 4$ block $B$ do the following: do

(a) Approximate B to a $L 4 \times M 4$ DCT block as follows:

$$
\hat{\mathbf{B}}^{(L 4 \times M 4)}=\left[\begin{array}{cc}
\sqrt{L M} \mathbf{B} & \mathbf{Z}_{(4,(M-1) 4)} \\
\mathbf{Z}_{((L-1) 4,4)} & \mathbf{Z}_{((L-1) 4,(M-1) 4)}
\end{array}\right]
$$

where $\mathbf{Z}_{(a, b)}$ denotes a matrix of $a \times b$ zero elements.

(b) Decompose $\hat{\mathrm{B}}^{(L 4 \times M 4)}$ into $L \times M$ number of $4 \times 4$ DCT blocks as follows.

$$
\left[\begin{array}{cccc}
\mathbf{B}_{0,0}^{(4 \times 4)} & \mathbf{B}_{0,1}^{(4 \times 4)} & \ldots & \mathbf{B}_{0, M-1}^{(4 \times 4)} \\
\mathbf{B}_{1,0}^{(4 \times 4)} & \mathbf{B}_{1,1}^{(4 \times 4)} & \ldots & \mathbf{B}_{1, M-1}^{(4 \times 4)} \\
\ldots \ldots \ldots & \ldots \ldots \ldots \ldots & \ldots \ldots \ldots \\
\mathbf{B}_{L-1,0}^{(4 \times 4)} & \mathbf{B}_{L-1,1}^{(4 \times 4)} & \ldots & \mathbf{B}_{L-1, M-1}^{(4 \times 4)}
\end{array}\right]=\mathbf{A}_{(L, 4)}^{T} \hat{\mathbf{B}}^{(L 4 \times M 4)} \mathbf{A}_{(M, 4)}
$$

end for

As in the adapted IDDA and IDAD for doubling H.264/AVC frames, we simplified the decomposition step. Because the conversion matrix $\mathbf{A}_{(L, 4)}$ is orthonormal, we altered the original equation $\mathbf{A}_{(L, 4)}^{-1} \hat{\mathbf{B}}^{(L 4 \times M 4)} \mathbf{A}_{(M, 4)}^{-1^{T}}$ to the one expressed in Equation (4.22).

\subsection{Resizing with Arbitrary Factors}

Resizing images by arbitrary factors are expressed in the form of $\frac{P}{Q} \times \frac{R}{S}$, where $P, Q, R$ and $S$ are positive integers. An approach to resize images by arbitrary factors is to first upsample an image by integral factor $P \times R$ followed by downsampling operation by integral factor of $Q \times S$. Mukherjee and Mitra [13] proposed this approach by using LMUS for the upsampling and LMDS for the downsampling operation. Mukhopadhyay [14] referred this approach as UD-Resizing algorithm (UDRA). They also proposed to employ LMDS and LMUS in the reverse order of UDRA, where downsampling using LMDS is first applied to the image followed by upsampling using LMUS algorithm, which called DU-Resizing algorithm (DURA) by Mukhopadhyay 
[14]. In this thesis, we experimented with both approaches.

\subsection{Hybrid Approach}

There exists instances where resizing an image fails to fall under the category of downsampling or upsampling. For example, you may want to increase the width of an image but decrease its height. In that case, Mukhopadhyay [14] referred to this operation as hybrid approach. Mukhopadhyay developed a Hybrid Resizing Algorithm (HRA) following a computational sequence of first block composition, subband approximation and block decomposition. However, the subband approximation is written in a more general form as well as the DCT block size. The algorithm will work on images composed of DCT blocks whose size could be $8 \times 8$ or $4 \times 4$. In this thesis, HRA was implemented based on Algorithm 7. Appendix E displays the algorithm written in the $\mathrm{C} / \mathrm{C}++$ programming language.

\subsection{Summary}

H.264/AVC departs from previous video compression standards by employing $4 \times 4$ integer transform to create blocks of $4 \times 4$ DCT coefficients. All of these algorithms except for HRA outlined in this chapter had to be modified to work with this compressed representation. The next chapter will describe how we implemented these algorithms. 


\section{Chapter 5}

\section{General Description of Experimental Environment}

\subsection{Introduction}

This chapter outlines the general setup that is common to most of our experiments, which will be covered in subsequent chapters.

\subsection{The Image Sample Set and H.264/AVC File Representation}

For the experiments, to represent one video frame in a H.264/AVC video bitstream, we created binary files from raw 8-bit greyscale $512 \times 512$ image files. See Appendix $\mathrm{D}$ for the list of images used in the experiments. We implemented an encoder that performed the default H.264/AVC forward transform as outlined in Equation (2.11). The encoder transformed raw image files to files representing one H.264/AVC frame composed of blocks of $4 \times 4$ DCT coefficients. Because H.264/AVC transform process includes quantization and scaling, and it is difficult to separate these steps from the transform process, we set the quantization parameter (QP) at a constant value of 6 for most experiments unless stated otherwise. We created a decoder application that implemented the default H.264/AVC inverse transform based on Equation (2.12). Both encoder and decoder application were written in $\mathrm{C} / \mathrm{C}++$ programming language using Microsoft Visual $\mathrm{C}++2010$ compiler.

\subsection{The DCT Domain H.264/AVC Frame Resize Algorithms}

We implemented the following resizing algorithms for image quality and computational cost:

IHS Image Halving through bilinear interpolation in the Spatial domain.

IDS Image Doubling through zero-order interpolation in the Spatial domain. 
IHBI [7][11] Image Halving through Bilinear Interpolation.

IDZOI [7][11] Image Doubling through Zero-Order Interpolation.

IHFDS [12] Image Halving through Low-Pass Filtering followed by Downsampling.

IDUSZP [12] Image Doubling through Upsampling followed by Zero-Padding.

IHAC [13] Image Halving through Approximation followed by Composition.

IDDA [13] Image Doubling through Decomposition followed by Approximation.

IHCA [13] Image Halving through Composition followed by Approximation.

IDAD [13] Image Doubling through Approximation followed by Decomposition .

LMDS [13] $L \times M$ Downsampling Algorithm.

LMUS [13] $L \times M$ Upsampling Algorithm.

UDRA [13][14] UD-Resizing Algorithm.

DURA [13][14] DU-Resizing Algorithm.

HRA [14] Hybrid Resizing Algorithm.

Resizing algorithms were implemented as console applications written in $\mathrm{C} / \mathrm{C}++$ programming language. Interprocess communication between encoder, algorithm and decoder applications were accomplished through file passing and pipes.

We implemented all the DCT domain resizing applications based on the resized algorithms outlined in Chapter 4. All the resizing applications, even the spatial resizing implementations, read in a compressed image and output the resized image in a compressed format. The compressed format was H.264/AVC for both input and output image file. Both spatial resizing applications, IHS and IDS, employed the straight forward approach for its resizing process as shown in Figure 1.1. 


\subsubsection{C ++ Linear Algebra Library}

All of the resize algorithms depend on linear algebra so having a library that supported matrix data structures and operations was critical during development. NICTA ${ }^{1}$ developed a $\mathrm{C}++$ linear algebra library that provided a good balance between performance and ease of use, which they called Armadillo $^{2}$. People familiar with Matlab will discover its syntax to be very similar. Armadillo is open-source software and is distributed under a licence that is both open-source and commercial context friendly. We found Armadillo library delivered fast and reliable code in syntax pleasing manner. Appendix E shows an example of a function implemented using Armadillo matrix data structures and functions.

\subsection{PSNR Measurements}

A PSNR application was implemented and written in $\mathrm{C} / \mathrm{C}++$ programming language. The application reads two raw image files from console and computes the PSNR value based on Equation (2.14). The application outputs the PSNR value in dB onto console standard output.

Some of the resize algorithms may result in additional zero-padded rows and columns at the right and bottom margins of the output image. During the experiments, the PSNR application ignored these boundary blocks when PSNR values were computed.

\subsection{Computational Cost}

Each resize application calculates computational cost as per Equation 2.17 and outputs the results to console the number of multiplication and additions per pixel. In the case for downsampling applications, we used operations per pixel of the original image and for the case for upsampling applications, operations per pixel of the upsized image. Also, the conversion matrices used to resize the images were print out to console. This provided a way to visual verify and manually calculate the computational cost for each algorithm.

\footnotetext{
${ }^{1}$ NICTA http://nicta.com.au/

${ }^{2}$ Armadillo, $\mathrm{C}++$ Linear Algebra Library, http://arma.sourceforge.net/
} 


\subsection{Raw Image Viewer}

There exist several commercial and open-source raw image viewers, such as Irfan$V_{\text {View }}{ }^{3}$, which can display the resultant decoded image from a resize algorithm. However, these applications can only display one image at a time. One could open multiple instances of these applications so visual comparison can be carried out but it would be easier one application could display multiple images. So we developed an application, Raw Viewer, to allow us to view multiple raw images simultaneously. This application assisted in subjective validation of the resized images.

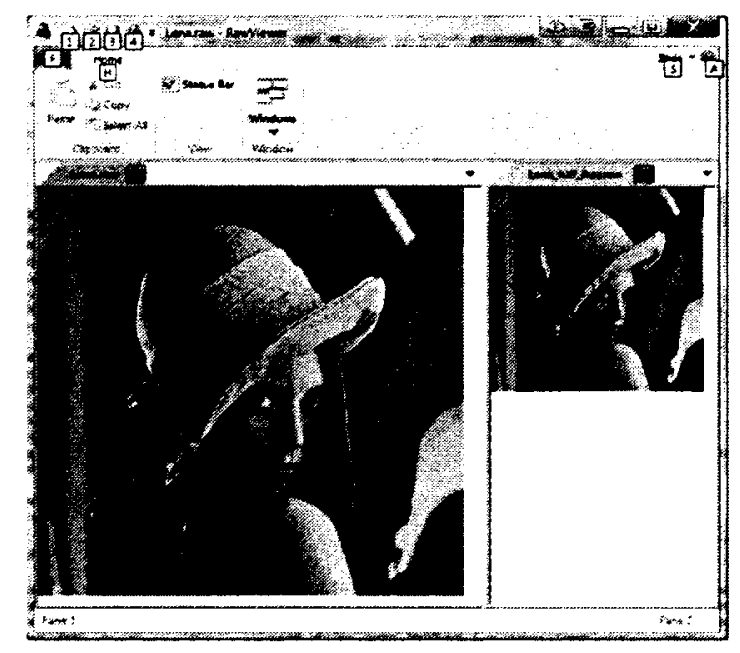

Figure 5.1: RawViewer application displaying the original Lena image on the left and Lena image halved on the right.

\subsection{Automation}

To compare and measure fifteen resize algorithms over a sample set of 27 images by hand would be tedious, error prone and time consuming. To automate the experimental process, we employed NMAKE ${ }^{4}$ application to control the inputs and outputs of the encoder, algorithms, decoder and PSNR application. NMAKE and their variants are used often in software development to build applications. In the context of the experiments, we used NMAKE to generate resized images and their measurements captured in text files.

\footnotetext{
${ }^{3}$ Irfanview, http://www.irfanview.ca/

${ }^{4}$ Microsoft NMAKE reference, http://msdn.microsoft.com/en-us/library/dd9y37ha.aspx
} 


\subsection{MATLAB for Resizing Images in the Spatial Domain}

Because we are using PSNR to measure image quality, we require a resized reference image to compute a PSNR when comparing an image generated from various resizing algorithms. MATLAB provides functionality to resize images in the spatial domain through the function imresize $e^{5}$. This function implements three interpolation methods, which are listed below:

nearest Nearest-neighbor interpolation; the output pixel is assigned the value of the pixel that the point falls within. No other pixels are considered.

bilinear Bilinear interpolation; the output pixel value is a weighted average of pixels in the nearest 2-by-2 neighborhood. This method produces less coding artifacts than nearest-neighbor in the final resized image [24].

bicubic Bicubic interpolation; the output pixel value is a weighted average of pixels in the nearest 4-by-4 neighborhood. Usually provides the best image quality.

All the methods perform anti-aliasing when an image is downsampled, except for nearest-neighbor.

\subsection{Summary}

For the experiments, binary files to represent H.264/AVC frames were encoded from raw binary files. The resize algorithms were implemented to resize these sample H.264/AVC files that were based on the algorithms outlined in Chapter 4. To measure image quality, we developed an application to read two raw image files and compute the PSNR value. The application, RawViewer, was created so one can view the results of the resizing algorithms. NMAKE assisted us to automate experimental runs, which saved us time and reduced human errors. Because we were using PSNR to measure image quality, we depended on MATLAB to create resized reference images. The next chapter will present the results of various experiments that we performed.

\footnotetext{
${ }^{5}$ MATLAB imresize reference, http://www.mathworks.com/help/images/ref/imresize.html
} 


\section{Chapter 6}

\section{Experimental Results from Resizing H.264/AVC Frames in the DCT Domain}

\subsection{Introduction}

In Chapter 4 , it was shown how we modified $8 \times 8 \mathrm{DCT}$ resize algorithms to resize H.264/AVC frames. In this chapter, we are interested in evaluating the performance of these adapted algorithms. One experimental objective is to evaluate the image quality generated from these DCT domain resizing algorithms by comparing them with the original image or an image resized in the spatial domain. Also, determining the computational cost of these algorithms is another important objective. In some case, we are interested in these algorithms' computational cost when comparing them with an approach carried out in the spatial domain. In other case, we compared the DCT resizing algorithms with each other. This chapter will first present image quality of the various adapted resizing algorithms followed by a section on the computational cost.

\subsection{Image Quality}

In this section, image quality of the various resized algorithms is presented. Six experiments were conducted to determine image quality performance. Some of the experimental tables were very large and are located in the appendix.

\subsubsection{Image Halving and Doubling Algorithms Performance}

Evaluating the algorithms performance for image quality, three experiments were developed for image halving and doubling algorithms, which are covered in the following subsections. 
Table 6.1: Experiment 1. A summary of the mean PSNR values for each halving algorithm.

\begin{tabular}{c|c|c|c|c|c}
\hline \hline & IHS & IHFDS & IHAC & IHBI & IHCA \\
\hline \hline mean(PSNR) & 43.5 & 43.10 & 43.01 & 41.36 & 40.62 \\
difference in dB & 0 & -0.40 & -0.49 & -2.14 & -2.88 \\
\hline \hline
\end{tabular}

\section{Image Halving Experiment}

In the first experiment, for each image in the sample set, an image $I_{\text {orig }}$ of size $N \times N$ is first spatially downsampled to an image $I_{d}$ of size $N / 2 \times N / 2$, which provided a reference image so PSNR can be computed when comparing images produced from the different image halving algorithms. We used MATLAB bicubic interpolation with anti-aliasing to halve the original image to create the downsampled image $I_{d}$ for the best image quality possible.

We evaluated five resizing algorithms; one spatial (IHS) and four DCT domain (IHBI, IHFDS, IHAC, IHCA). In this experiment, the original image $I_{\text {orig }}$ is first compressed using our H.264/AVC encoder to create compressed image file. Each resizing application read the newly created compressed image file and outputted a compressed halved image file. We then used our H.264/AVC decoder to transform the compressed halved image file to the spatial domain where a PSNR was computed with the reference image $I_{d}$.

The experimental results are outlined in Table A.1. The spatial resizing algorithm, IHS, provided the highest PSNR value but IHFDS and IHBI only suffered less than $0.50 \mathrm{~dB}$ loss. Figure 6.1 displays images generated from these halving algorithms. Subjectively, the image quality from all the resizing algorithms was judged to be close. Table 6.1 displays the mean PSNR values for each of the halving algorithm.

As outlined in Chapter 5, IHS application performed the full straight forward transcoding process as outlined in Figure 1.1. The IHS applications employed a simple bilinear interpolation without anti-aliasing when halving an image. We implemented this application because we can determine the computational cost for the full resizing process. If we depended on a third party implementation for halving, the resizing cost would be hidden from us. 


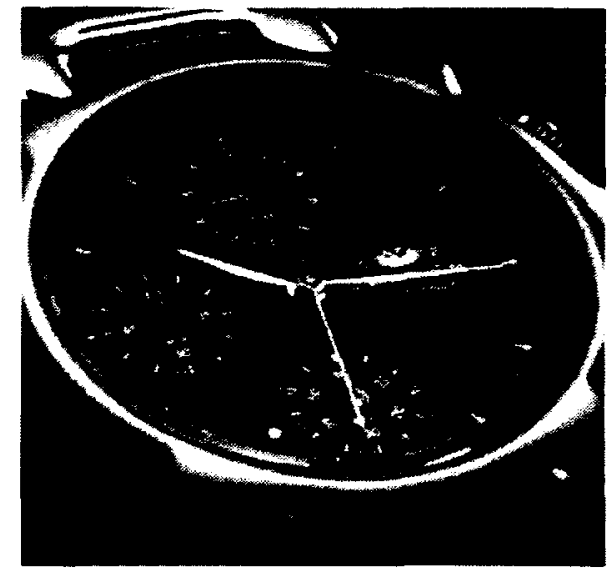

(a) IHS

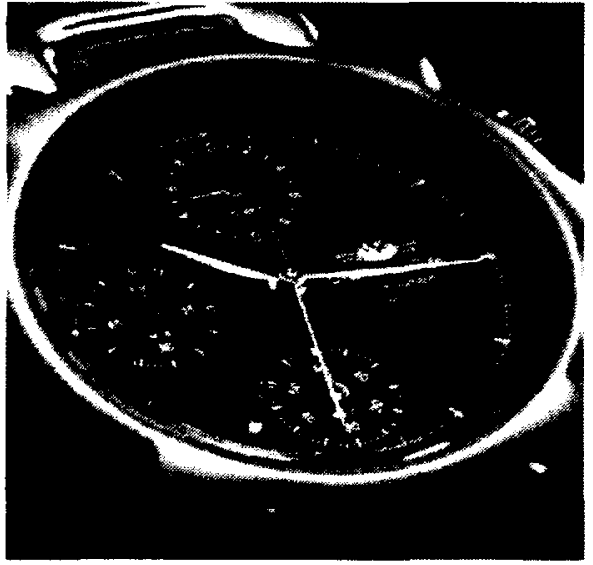

(b) IHBI

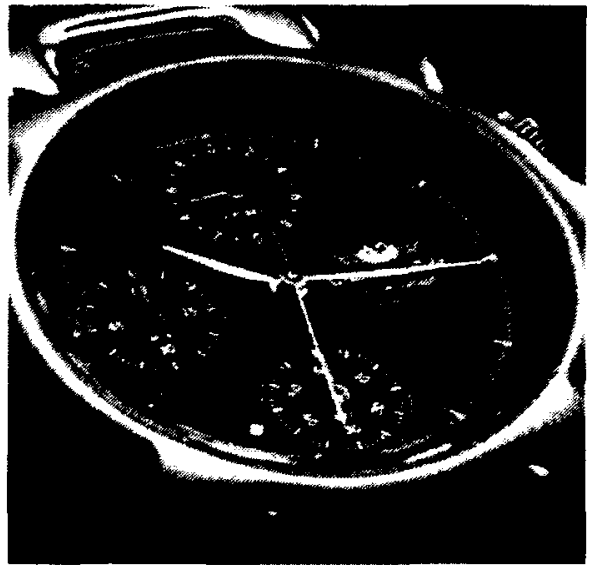

(d) IHFDS

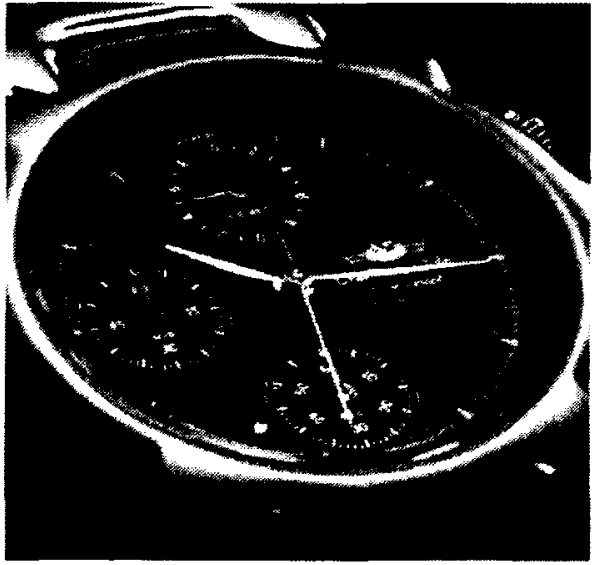

(c) IHAC

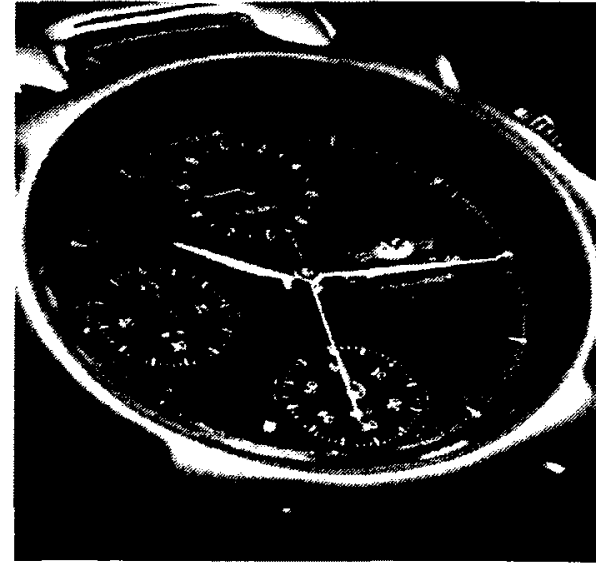

(e) IHCA

Figure 6.1: Figure 6.2(a) is an image half in the spatial domain using bilinear interpolation with PSRN value of 42.6. Figure 6.1(b) IHBI, Figure 6.1(c) IHAC, Figure 6.1(d) IHFDS and Figure 6.1(e) IHCA. The PSNR values are 38.79, 40.51, 40.57, and 36.53 respectively. 
Table 6.2: Experiment 2. A summary of the mean PSNR values for each doubling algorithm and difference in $\mathrm{dB}$.

\begin{tabular}{c|c|c|c|c|c}
\hline \hline & IDAD & IDDA & IDUSZP & IDS & IDZOI \\
\hline \hline mean(PSNR) & 31.62 & 31.08 & 31.08 & 29.89 & 29.42 \\
difference in dB & 0 & -0.54 & -0.54 & -1.96 & -2.20 \\
\hline \hline
\end{tabular}

\section{Image Doubling Experiment}

To evaluate image quality performance of the image doubling algorithms, spatially downsampled images $I_{d}^{s}$ are compressed then upsampled. We used MATLAB bicubic interpolation with anti-aliasing to halve the original image. We used our H.264/AVC encoder to compress the spatially downsampled images $I_{d}^{s}$. Later these compressed halved images are read in by the resizing algorithms, which outputted a compressed doubled image. Our H.264/AVC decoder would transform these compressed doubled images into the spatial domain. Then PSNR values were computed between the upsampled images with the original images $I_{\text {orig }}$.

Table A.2 displays the results of this experiment. We evaluated five resizing algorithms: one spatial (IDS) and four DCT domain (IDZOI, IDUSZP, IDDA, IDAD). The IDAD had the highest PSNR value, while IDDA and IDUSZP suffered approximately $0.54 \mathrm{~dB}$ loss. Table 6.2 outlines the mean PSNR value for each image doubling algorithm and their difference from the best in $\mathrm{dB}$.

Interestingly, while the spatial algorithm performed the best in halving experiment, the spatial doubling algorithm performed the worst. We believed the spatial doubling method used in the IDS algorithm greatly contributed to the distortion. In IDS, we implemented a simple zero-order interpolation for our spatial doubling algorithm. This method is known to produce visible coding artifacts in the resultant resized image [24]. Similarly, IDZOI also employed a simple zero-order interpolation but in the DCT domain, thus inheriting the same shortcomings as its spatial domain version.

IDS is similar IHS as it implemented the full straight forward resizing process as shown in Figure 1.1. The reason for implementing this spatial resizing algorithm followed the same reason for developing IHS. We needed a spatial doubling algorithm where the full computational cost can be determined. 


\section{Image Halving followed by Image Doubling Experiment}

In the third experiment, an image is first halved and then doubled. The resulting upsampled image is compared with the original image $I_{\text {orig }}$. We paired these halving and doubling algorithms for the following reasons:

IHS-IDS These are spatial resizing algorithms that implement the full straight forward resizing process as shown in Figure 1.1.

IHBI-IDZOI These are DCT resizing versions of the spatial algorithms, IHS-IDS.

IHAC-IDDA Both of these algorithms employed an $\mathbf{A}_{(2,2)}$ conversion matrix in their composition/decomposition step.

IHFDS-IDUSZP Complimentary halving and doubling algorithms that were developed by Dugad et al [12].

IHCA-IDAD Both of these algorithms employed an $\mathbf{A}_{(2,4)}$ conversion matrix in their composition/decomposition step.

In this experiment, we first compressed the original image $I_{\text {orig }}$ using our H.264/AVC encoder, which is then read in by the halving algorithms for each pairing. The halving applications outputted a compressed halved image that the doubling algorithms read in and output a compressed upsampled image in the original resolution. Our H.264/AVC decoder transformed the compressed doubled image back into the spatial domain where it is compared with the original image $I_{\text {orig }}$ using PSNR.

The PSNR values obtained after image halving followed by image doubling are outlined in Table A.3. In Table 6.3, the DCT domain resizing algorithms were compared with IHS-IDS algorithms. Only IHBI-IDZOI pairing had a lower mean PSNR value than IHS-IDS pairing. The remaining DCT domain resizing pairings produced greater mean PSNR values with the IHCA-IDAD pairing generating $2.19 \mathrm{~dB}$ gain over the spatial approach implemented by IHS-IDS pairing.

Implementing IHS-IDS with sub-optimal spatial resizing techniques contributed to lower PSNR values. IHS-IDS traded off image quality for better computational cost. The IHCA-IDAD algorithms employed $\mathbf{A}_{(2,4)}$ in their resizing algorithms, which utilized all the coefficients in the input image during resizing. This improved image 
Table 6.3: Experiment 3. A summary of the mean PSNR values after image halving followed by image doubling and difference in $\mathrm{dB}$ from IHS-IDS resizing.

\begin{tabular}{c|c|c|c|c|c}
\hline \hline & IHS & IHBI & IHAC & IHFDS & IHCA \\
& IDS & IDZOI & IDDA & IDUSZP & IDAD \\
\hline \hline mean(PSNR) & 30.05 & 29.69 & 31.34 & 31.34 & 32.24 \\
difference in dB & 0 & -0.36 & +1.29 & +1.29 & +2.19 \\
\hline \hline
\end{tabular}

quality because high frequency coefficients are included in the resizing operation but increased computational cost. The IHAC-IDDA and IHFDS-IDUSZP decimated the DCT coefficients and only use the low frequency coefficients during resizing. This lowered computational cost because the algorithms only operated on a sub-set of the coefficients; thus, reduced matrix multiplications and additions. However, this increased distortion in the final resized image because the high-frequency coefficients are removed during resizing.

\subsubsection{Integral Resizing Algorithms}

Experiment 4 compares LMDS and LMUS resize algorithms by downsampling and upsampling with a variety of scaling factors $L \times M$. A similar approach was followed that was conducted in the image halving and doubling experiment. The first approach computed the PSNR between the downsampled image $I_{d}^{c}$ using LMDS algorithm with an image $I_{d}^{s}$ produced by bilinear resizing operation in the spatial domain. In Table B.1, these PSNR values are referred as DS-PSNR. The second approach was to upsample the spatial downsampled image $I_{d}^{s}$ and compute the PSNR with respect to the original image $I_{\text {orig }}$. These PSNR values are referred in Table B.1 as US-PSNR. In the third approach, the image $I_{\text {orig }}$ was downsampled using LMDS algorithm followed by upsampling using the LMUS algorithm to create the resulting image. This resulting image is compared with the original image $I_{\text {orig }}$ to obtain a PSNR value. The last approach, images were downsampled followed by upsampling the original image $I_{\text {orig }}$ in the spatial domain using bilinear resizing algorithm. Then the resulting image was compared with original image $I_{\text {orig }}$ to derive a PSNR value. Both approaches will be referred as DS-US-PSNR in Table B.1. When comparing downsample followed by upsample experiments, in all cases the LMDS-LMUS performed 1 to $2 \mathrm{~dB}$ better than the spatial bilinear approach. 


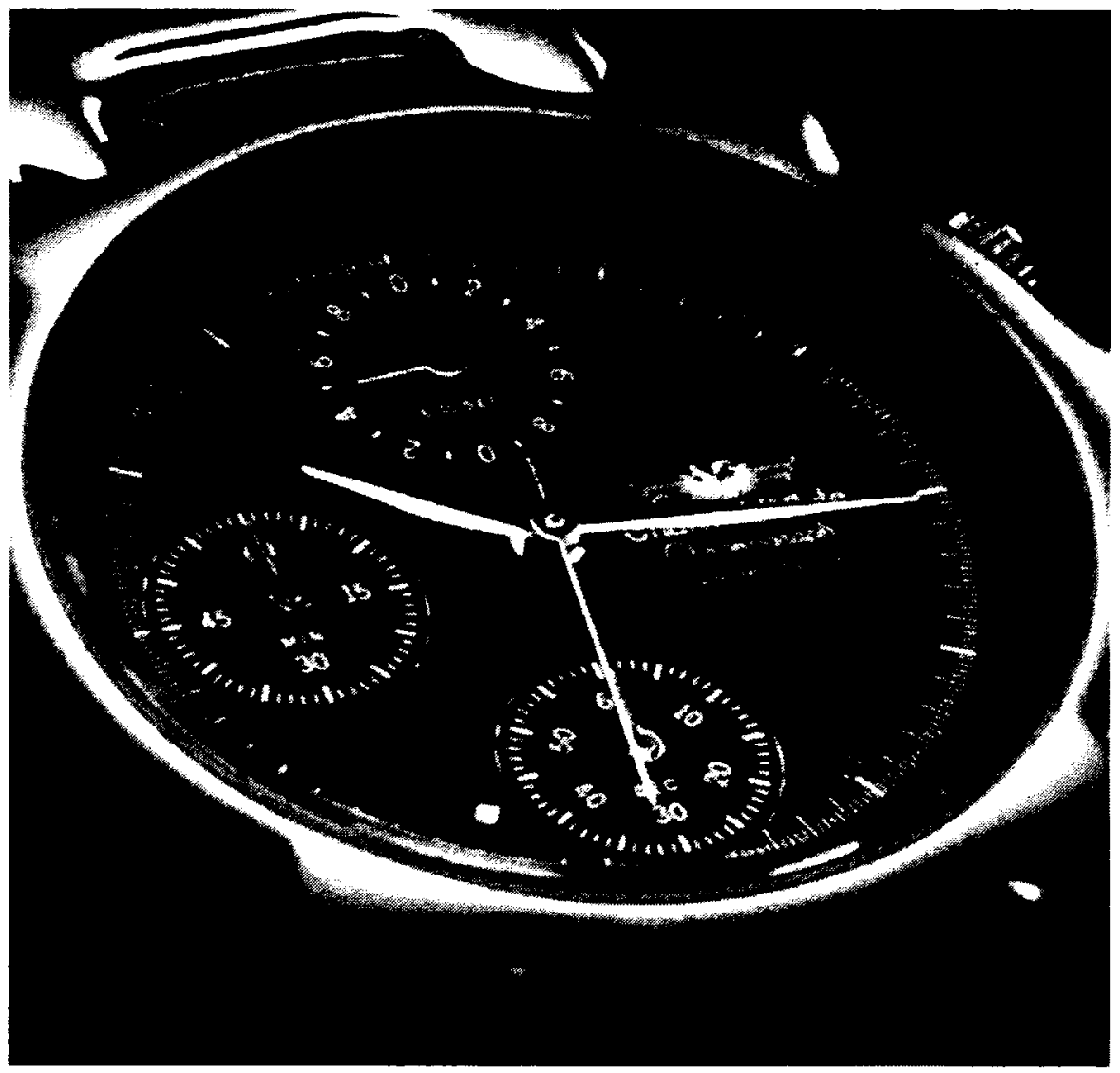

(a) Original

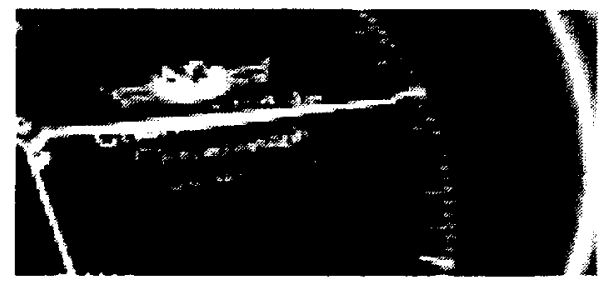

(b)

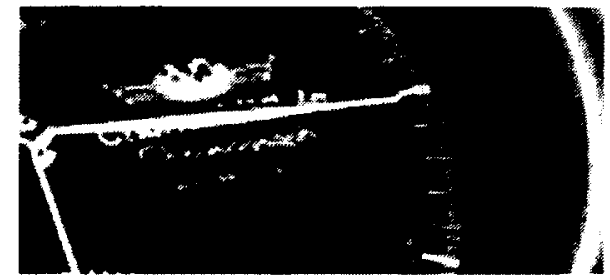

(e)

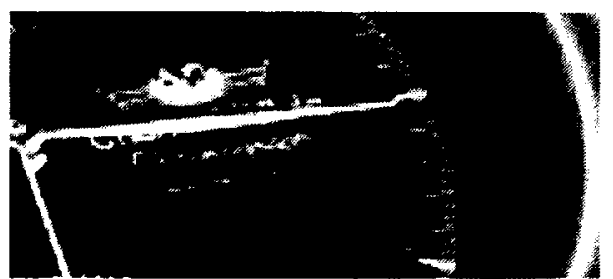

(c)

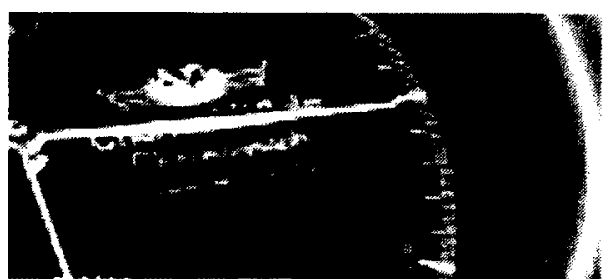

(d)

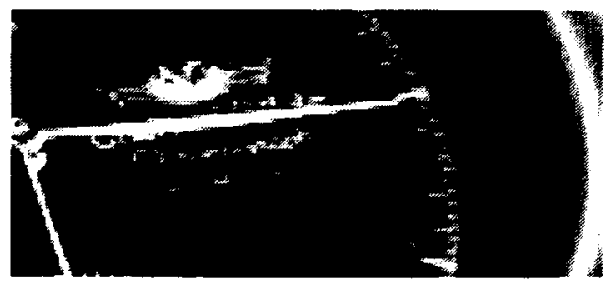

(f)

Figure 6.2: Watch image: 6.2(a) Original. Portion of the resultant images from experiment 3, where images were first halved then doubled: 6.2(b) Spatial resizing; 6.2(c) IHAC-IDDA, 6.2(d) IHFDS-IDUSZP, 6.2(e) IHCA-IDAD, 6.2(f) IHBI-IDZOI. The PSNR values with respect to the original are $25.98,27.69,27.67,29.14$ and 25.51 $\mathrm{dB}$ respectively. 


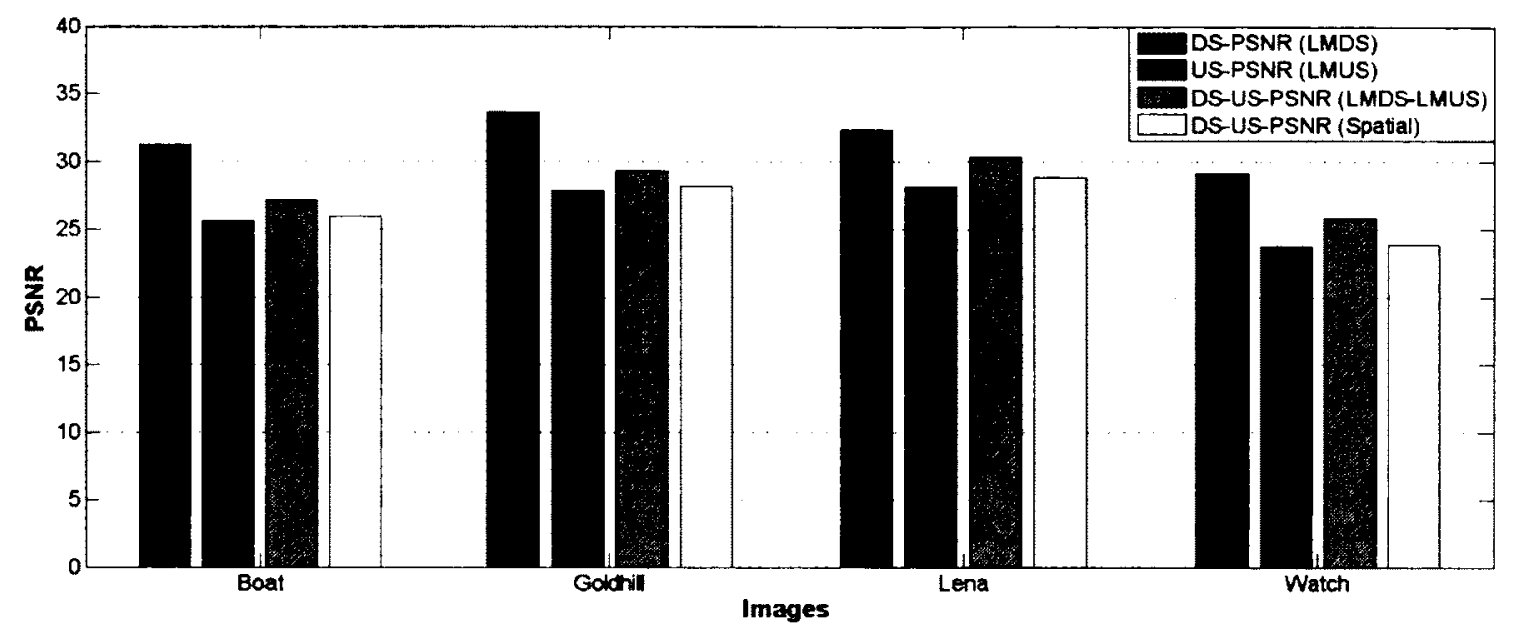

Figure 6.3: A chart showing the mean PSNR value for each image when scaled by various factors $L \times M$ based on experiment 4 .

\subsubsection{Arbitrary Resizing Algorithms}

Two experiments were developed to evaluate image quality generated from resizing algorithms with arbitrary factors, which are covered in the following subsections.

\section{Downsampling by Arbitrary Factors Experiment}

Experiment 5 compares HRA, DURA and UDRA algorithms by resizing using a variety of scale factors $\frac{P R}{Q S}$. PSNR values were obtained by comparing the resized image $\left(I_{r}^{c}\right)$ with a spatial resized image $\left(I_{r}^{s}\right)$ and are outlined in Table C.1. The PSNR values were computed by comparing the resized image with an image produced by bilinear operation in the spatial domain. In this experiment, URDA produced images with PSNR value that was slightly better than HRA. DURA produced images with PSNR value significantly worse than both HRA and UDRA.

\section{Downsampling followed by Upsampling by Arbitrary Factors Experiment}

Experiment 6 is similar to experiment 3. The original image is arbitrary resized followed by inverse resized operation to return the image to its original resolution. PSNR values were computed by comparing the final resized image with the original image $I_{\text {orig. }}$ In all cases, HRA produced images with a higher PSNR value than 


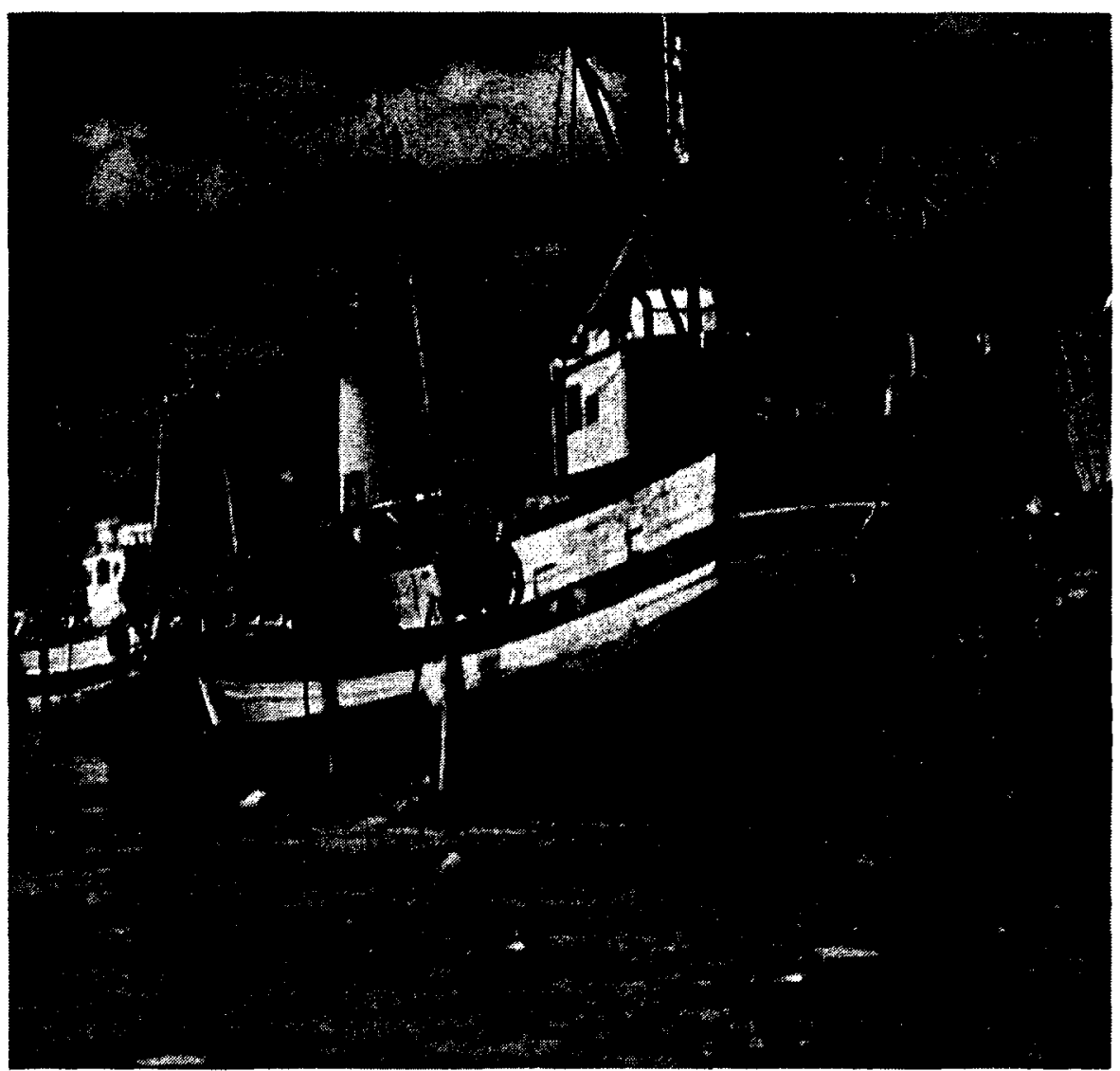

(a) Original

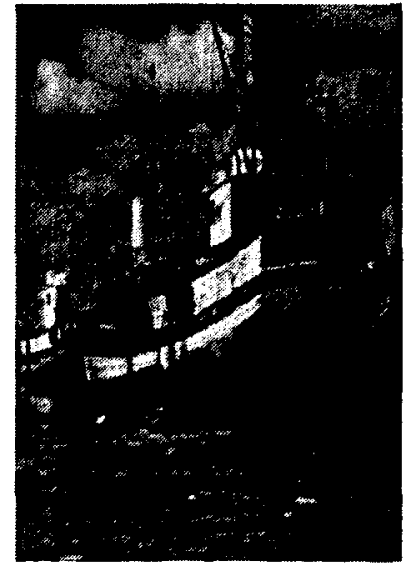

(b) $2 \times 3$

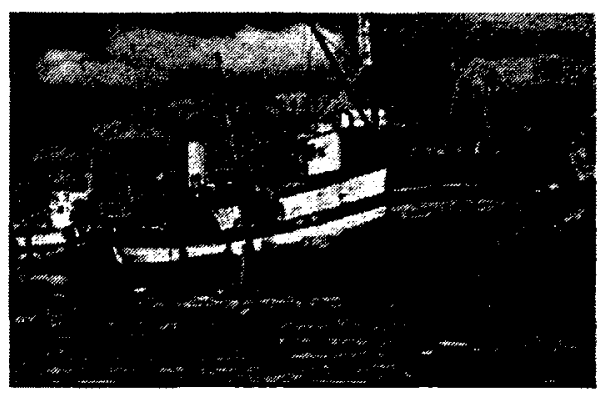

(c) $3 \times 2$

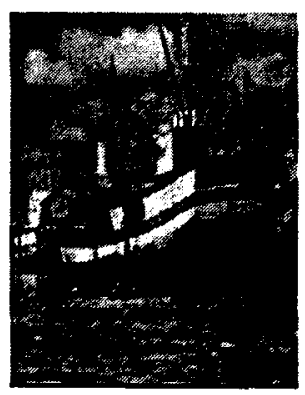

(d) $3 \times 4$

Figure 6.4: Images generated from applying LMDS algorithm. Figure 6.4(a) displays the original image. Figure $6.4(\mathrm{~b})$ is an image scaled by a factor of $2 \times 3$. Figure $6.4(\mathrm{c})$ is scaled by $3 \times 2$ and Figure $6.4(\mathrm{~d})$ by $3 \times 4$. The computed PSNR values when compared with an image resized in the spatial domain is $30.71,30.36$ and $30.58 \mathrm{~dB}$ respectively. 


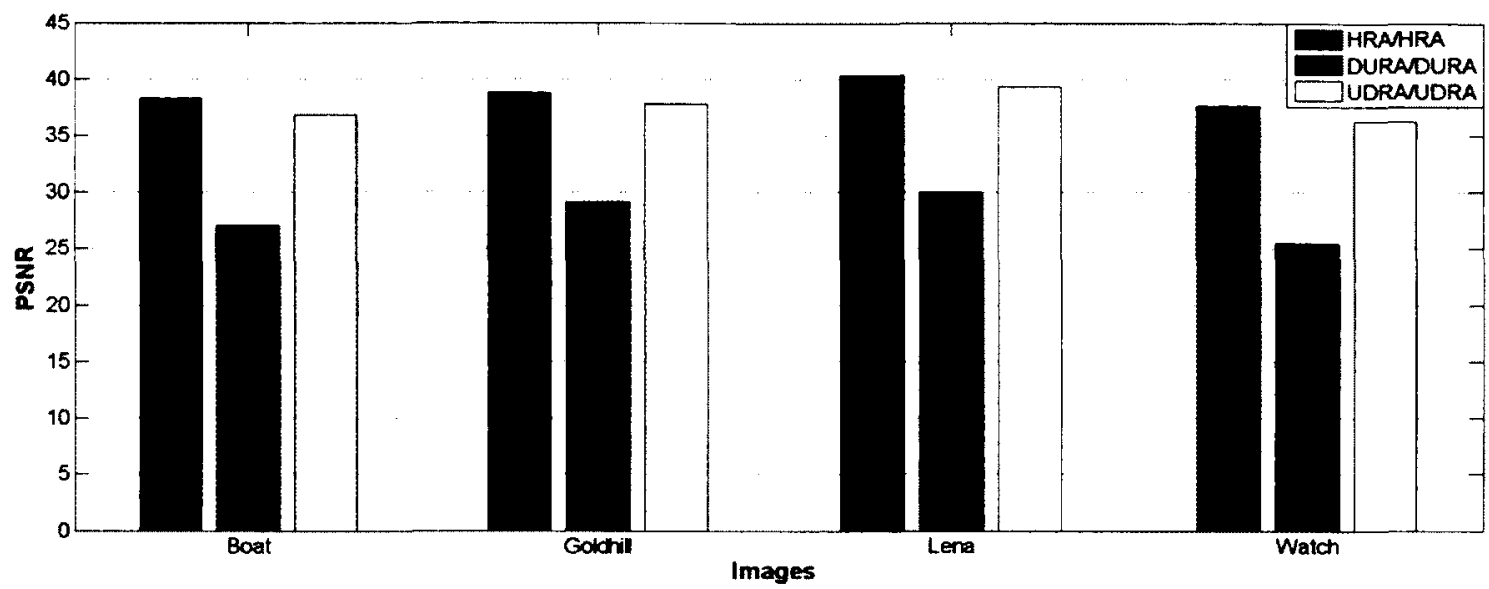

Figure 6.5: A summary of the mean PSNR values $(\mathrm{dB})$ that was computed over a sample set obtained in experiment 6 , where the image was first resized by arbitrary factor followed by inverse resize operation to return the image to its original resolution.

UDRA and DURA. The DURA method performed the worst and produces images with noticeable blocking artifacts. Figure 6.6 displays sample images produced from these algorithms.

\subsection{Computational Cost}

In this section, computational cost for each resize algorithm is presented. The computational costs are expressed as the number of multiplications $\left(n_{m}\right)$ and additions $\left(n_{a}\right)$. Also, we used a ratio of PSNR per computational cost to determine how much does it cost to produce a number of dB's. The higher the ratio indicates better efficiency because algorithm is using fewer operations to produce a given number of dB's. This ratio will be expressed as follows:

$$
q u a l i t y / \text { cost }=\frac{\operatorname{mean}(P S N R)}{\left(n_{m}+n_{a}\right)}
$$

The mean $(P S N R)$ is average PSNR over a sample set of computed PSNR values produced from a given resize algorithm. Using this ratio of quality/cost, will assist in evaluating algorithms with respect with each other. 


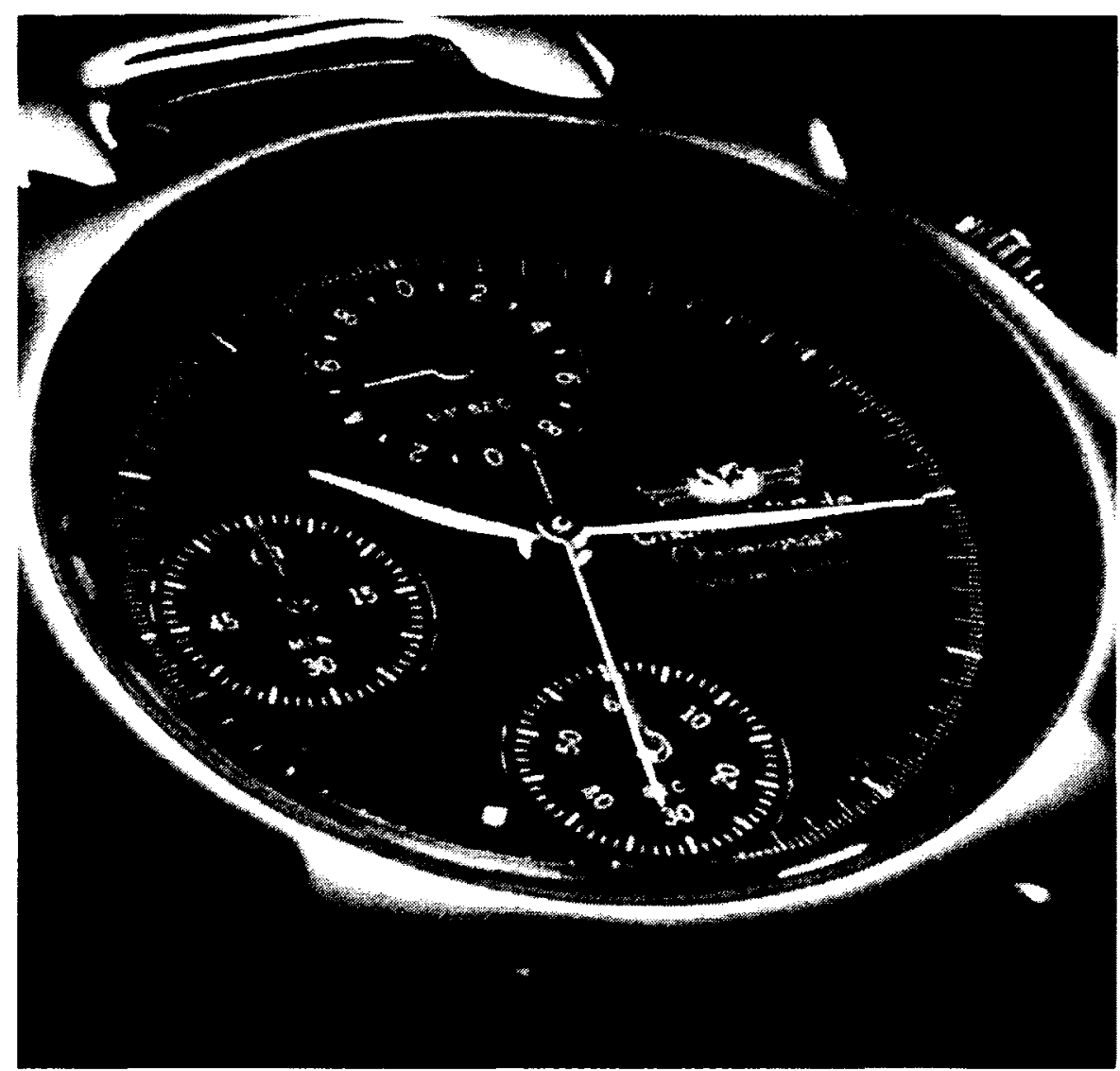

(a) Original

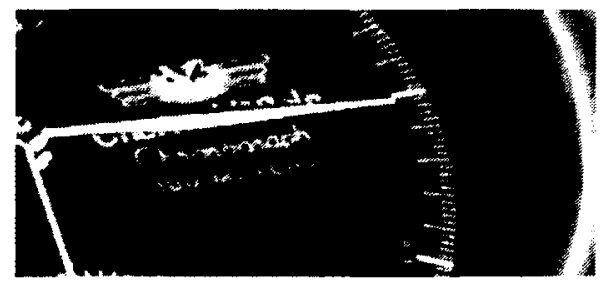

(b)

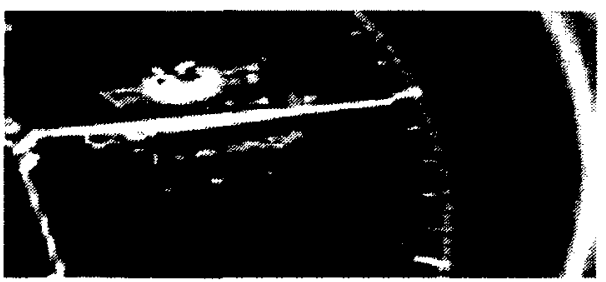

(c)

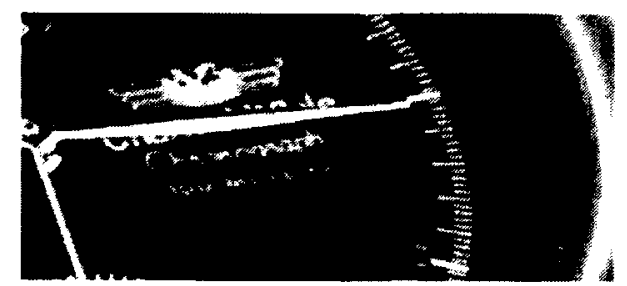

(d)

UDRA

Figure 6.6: Sample images produced from experiment 6, where images were arbitrary resizing by a factor $\frac{3}{2} \times \frac{5}{4}$ followed by inverse resizing. Figure 6.6(a) is the original image. Notice DURA created image displayed in Figure 6.6(c) suffers from blocking artifacts. 


\subsubsection{Computational Cost for Image Halving and Doubling Resize}

\section{Algorithms}

This subsection outlines computational cost for image halving and doubling resize algorithms. Table 6.4 displays the results for image halving algorithms. The image halving algorithm with the lowest computational cost was IHAC with $n_{m}=0.5$ and $n_{a}=1$ which was significantly less than a comparable operation in the spatial domain implemented by the IHS algorithm. IHAC also had the best quality/cost ratio of 28.67. IHBI, IHFDS and IHAC provided a better quality/cost ratio than spatial domain resizing. We determined that $\mathrm{IHAC}$ was best image halving algorithm that provided an acceptable image quality with low computational cost.

Table 6.4: Computation cost of image halving algorithms per pixel of the original image

\begin{tabular}{c|c|c|c|c|c}
\hline \hline ops & IHS & IHBI & IHFDS & IHAC & IHCA \\
\hline \hline mean(PSNR) & 43.5 & 41.36 & 43.1 & 43.01 & 40.62 \\
\hline$n_{m}$ & 1.25 & 4.5 & 2 & 0.5 & 4 \\
\hline$n_{a}$ & 8.25 & 2.5 & 1 & 1 & 8 \\
\hline \hline quality $/$ cost & 4.58 & 5.91 & 14.37 & 28.67 & 3.39 \\
mean $(P S N R) /\left(n_{m}+n_{a}\right)$ & & & & & \\
\hline \hline
\end{tabular}

Table 6.5 displays the results for image doubling algorithms. For image doubling algorithms IDUSZP had the lowest computational cost while IDAD had the greatest. IDUSZP and IDDA were close in quality/cost ratio of 10.36 and 8.88 respectively and have greater quality/cost ratios than upsampling in the spatial domain using the IDS algorithm. In the previous section on image quality, both IDUSZP and IDDA produced on average higher PSNR than IDZOI except for IDAD which was highest average PSNR value but with significantly lower quality/cost ratio of 1.73 . In this case, we have two image doubling algorithms that produce images of good quality with low computational cost, IDUSZP and IDDA. 
Table 6.5: Computation cost of image doubling algorithms per pixel of the upsampled image

\begin{tabular}{c|c|c|c|c|c}
\hline \hline ops & IDS & IDZOI & IDUSZP & IDDA & IDAD \\
\hline \hline mean(PSNR) & 29.89 & 29.42 & 31.08 & 31.08 & 31.62 \\
\hline$n_{m}$ & 1.25 & 4 & 2 & 2 & 10 \\
\hline$n_{a}$ & 8.25 & 2.5 & 1 & 1.5 & 8 \\
\hline \hline $\begin{array}{c}\text { quality/cost } \\
\text { mean }(P S N R) /\left(n_{m}+n_{a}\right)\end{array}$ & 3.15 & 4.53 & 10.36 & 8.88 & 1.76 \\
\hline \hline
\end{tabular}

The spatial resizing algorithms IHS and IDS employed sub-optimal resizing methods. We selected these resizing methods because calculating the computation cost was straight forward. We believed implementing a more optimal resizing method for these algorithms would increase image quality but would increase computation cost. Therefore, the quality/cost ratio may remain constant. This will have to be confirmed through future experiments.

\subsubsection{Computational Cost for Resizing Algorithms with Integral Factors}

In this thesis, LMDS was developed to downsample H.264/AVC frame by arbitrary factors and for the inverse operation, LMUS was implemented. In these implementations, full composition and decomposition was carried out. In Mukherjee and Mitra paper [21], they proposed a method to reduce matrix operations by using only the submatrices of the composition and decomposition matrix. This is very similar technique that Dugad and Ahuja employed with their filter matrix, where only the low frequency coefficients are used during the resizing operation. We plan to implement these improvements in future studies and compare them with non-optimized algorithms developed in this thesis.

Table 6.6 outlines the computational cost for the LMDS algorithm while Table 6.7 outlines the cost for LMUS algorithm. You may observe that upsampling a H.264/AVC frame is more expensive than downsampling. 
Table 6.6: LMDS Computational cost per pixel of the original image

\begin{tabular}{c|c||c|c}
\hline \hline$L \times M$ & $\begin{array}{c}\text { LMDS } \\
\left(n_{m}, n_{a}\right)\end{array}$ & $L \times M$ & $\begin{array}{c}\text { LMDS } \\
\left(n_{m}, n_{a}\right)\end{array}$ \\
\hline \hline $1 \times 2$ & $(4,8)$ & $3 \times 3$ & $(7.44,16.89)$ \\
$2 \times 2$ & $(4,8)$ & $3 \times 4$ & $(11,21)$ \\
$2 \times 3$ & $(7.33,12.67)$ & $3 \times 5$ & $(15.73,28)$ \\
$2 \times 5$ & $(20.8,37.6)$ & $4 \times 4$ & $(12,24)$ \\
$3 \times 2$ & $(7.33,12.67)$ & $5 \times 5$ & $(16,28.8)$ \\
\hline \hline
\end{tabular}

Table 6.7: LMUS computational cost per pixel of the upsampled image

\begin{tabular}{c|c||c|c}
\hline \hline$L \times \bar{M}$ & $\begin{array}{c}\text { LMUS } \\
\left(n_{m}, n_{a}\right)\end{array}$ & $L \times M$ & $\begin{array}{c}\text { LMUS } \\
\left(n_{m}, n_{a}\right)\end{array}$ \\
\hline \hline $1 \times 2$ & $(10,8)$ & $3 \times 3$ & $(15.33,13.33)$ \\
$2 \times 2$ & $(10,8)$ & $3 \times 4$ & $(23.08,21)$ \\
$2 \times 3$ & $(14.83,12.67)$ & $3 \times 5$ & $(30.27,28)$ \\
$2 \times 5$ & $(40.5,37.6)$ & $4 \times 4$ & $(26,24)$ \\
$3 \times 2$ & $(14.83,12.67)$ & $5 \times 5$ & $(30.8,28.8)$ \\
\hline \hline
\end{tabular}

\subsubsection{Computational Cost for Arbitrary Resizing Algorithms}

Three resize algorithms were compared for computational cost: HRA, DURA and UDRA. Table 6.8 outlines computational cost for each respective algorithm and the quality/cost ratio using different resize factors. Even though DURA has the highest quality/cost ratios it suffered greatly from the appearance of blocking artifacts. HRA provided a good compromise between cost and quality. It has a higher quality/cost ratio than UDRA and produced images with quality almost as par with those created by the later. 
Table 6.8: Computational cost of arbitrary resizing algorithms per pixel of the original image and the quality/cost ratio

\begin{tabular}{c|c|c|c}
\hline \hline \multirow{2}{*}{ Resizing Factor } & \multicolumn{3}{|c}{$\left(n_{m}, n_{a}\right.$, quality $/$ cost $)$} \\
\cline { 2 - 4 } & HRA & DURA & UDRA \\
\hline \hline$\frac{2}{3} \times \frac{4}{5}$ & $(22.67,41.62,0.49)$ & $(9.25,3.27,2.14)$ & $(138.75,49,0.17)$ \\
$\frac{3}{2} \times \frac{5}{4}$ & $(42.5,78.03,0.53)$ & $(17.8,10.05,2.36)$ & $(142.38,80.38,0.18)$ \\
$\frac{2}{3} \times \frac{3}{2}$ & $(14.67,25.33,0.53)$ & $(13.08,4.71,2.28)$ & $(78.5,28.25,0.18)$ \\
$\frac{3}{2} \times \frac{2}{3}$ & $(14.67,25.33,0.46)$ & $(13.02,5.27,2.20)$ & $(78.13,31.63,0.16)$ \\
\hline \hline
\end{tabular}

\subsection{Summary of Results}

Let $n_{m}$ represent the number of matrix multiplications and $n_{a}$ the number of matrix additions. Halving an H.264/AVC frame in the spatial domain has a computational cost of $n_{m}=1.25$ and $n_{a}=4.4375$ per pixel of the original image. Four image halving algorithms were implemented to reize H.264/AVC frames in the compressed domain. All the halving algorithms produced images with a mean PSNR greater than $40 \mathrm{~dB}$ but with different computational cost. The image halving algorithm, IHAC, was the most efficient with $n_{m}=0.5$ and $n_{a}=1$ per pixel of the original image and created images with a mean PSNR of $43.01 \mathrm{~dB}$.

The inverse operation of image doubling, four algorithms were developed. In an experiment where images were downsampled and upsampled, three of these algorithms produced images with greater mean PSNR values than comparable operations in the spatial domain. The IDAD, IDUSZP and IDDA produced images with an PSNR value greater than spatial domain resizing using the IDS algorithm. IDZOI, IDUSZP and IDDA had a lower computational cost than spatial domain resizing implemented by the IDS algorithm.

For resizing H.264/AVC frames by integral factors, two algorithms were developed: LMDS for downsampling and LMUS for upsampling. These algorithms are generalization of the IHCA and IDAD algorithms. Although IHCA and IDAD algorithms are more computational expensive than their counterparts, they are easier to adapt for resizing images by integral factors. In the image quality experiments, these 
algorithms produced higher PSNR values in all cases than equivalent spatial domain resizing using bilinear method.

With algorithms to resize images by integral factors, one can use LMDS and LMUS algorithms in tandem to create images of arbitrary factors. Depending the ordering of LMDS and LMUS operations, images can be created with lower or higher quality with lower or higher computational cost. The DURA algorithm applies the LMDS first followed by LMUS, while UDRA algorithm applies them in reverse order. UDRA produced images with higher PSNR than DURA but with a higher computational cost. Images created from DURA were observed to suffer heavily from blocking artifacts. Mukhopadhyay [14] proposed a hybrid resizing algorithm (HRA) following a computational sequence of first block composition, subband approximation followed by block decomposition. However, the subband approximation is written in a more general form. Even though HRA was less efficient than DURA it produced images with PSNR values similar to UDRA at a lower cost. 


\section{Chapter 7}

\section{Removing Quantization Step in the DCT Domain Resizing Process}

\section{$7.1 \quad$ Introduction}

Chapter 2 provided an overview of H.264/AVC standard. This standard has an unique coding feature where the default forward and inverse transform process includes quantization and scaling. In other words, the transform and quantization steps overlap and it is not obvious when transform stage ends and quantization begins. Also, when transforming an image from the spatial to the frequency domain, H.264/AVC employs $4 \times 4$ integer transform, an approximate form of the DCT, to form blocks of $4 \times 4$ DCT coefficients.

As illustrated in Figure 7.1, a typical DCT domain resizing process includes the following steps: 1) entropy decoding, 2) inverse quantization, 3) DCT domain resizing, 4) forward quantization and 5) entropy encoding. Therefore, a method was required to de-quantize $4 \times 4$ DCT coefficients before DCT domain resizing and quantize $4 \times 4$ DCT coefficients after DCT domain resizing. A section in this chapter will cover an approach we developed to do forward and inverse quantization.

One of the research goals is to determine if we can remove the inverse and forward quantization steps from the default DCT domain resizing process as outlined in Figure 7.1 and create a new resizing process as illustrated in Figure 7.2. By removing the

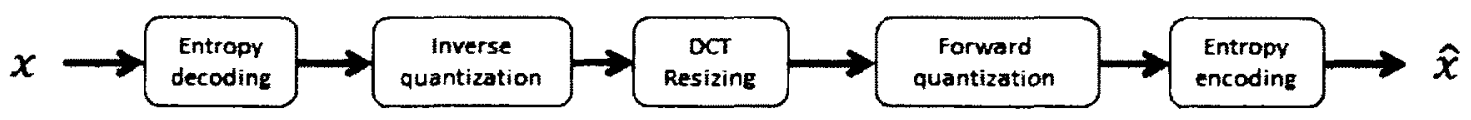

Figure 7.1: The processing flow of resizing images or video frame in the DCT domain, where $x$ is the original image and $\hat{x}$ is the resized image. 


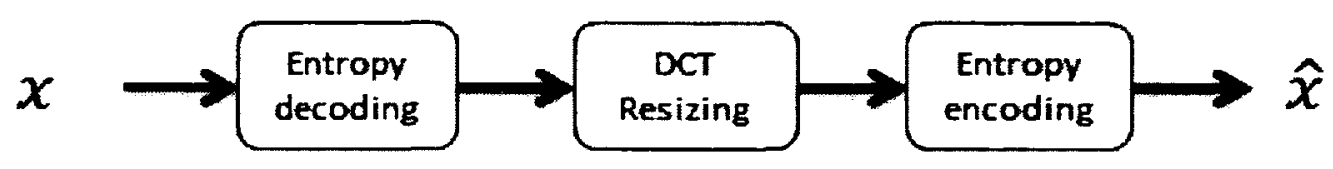

Figure 7.2: A resizing H.264/Frame without quantization step, where $x$ is the original frame and $\hat{x}$ is the resized frame.

quantization step, we can reduce the overall computational cost of the resizing process and hopefully not suffer any degradation in image quality.

We used two objectives to evaluate the performance of the new process as shown in Figure 7.2. First, we will compare image quality of the two processes. Second, we will measure bitrate efficiency between the two processes by entropy encoding the resized images. If the new process generates images of similar quality at an acceptable bitrate, then the new process could be recommended as a method to reduce the overall computational cost of image resizing.

\subsection{Creating Quantization Matrix}

Early in our research, we assumed that we had to apply inverse quantization on the DCT coefficients before DCT resizing. Also, after DCT resizing, the forward quantization would have to be applied on the resized image. The standard does not explicitly define a method to de-quantized H.264/AVC coefficients; therefore, we derived a method for our research. Our method creates an quantization matrix that includes scaling. We derived this quantization matrix, $\mathbf{Q}$, from the equation used to create the matrix $\mathbf{V}_{i 4}$, which is incorporated in both forward and inverse H.264/AVC transform process.

$$
\begin{aligned}
\mathbf{V}_{i 4} & =\mathbf{S}_{i 4} \cdot Q_{s t e p}[Q P] \cdot 2^{6} \\
\mathbf{Q} & =\frac{\mathbf{V}_{i 4}}{\mathbf{S}_{i 4} \cdot 2^{6}}
\end{aligned}
$$

Let $\mathbf{X}$ represent a $4 \times 4$ block of H.264/AVC coefficients. To de-quantize $\mathbf{X}$ you apply quantization matrix $\mathbf{Q}$ on $\mathbf{X}$ as follows to get $\mathbf{Y}$.

$$
\mathbf{Y}=\mathbf{X} \bullet \mathbf{Q}
$$


where operator $\bullet$ denotes an element-by-element multiplication.

For forward quantization, $\mathbf{Q}$ is divided into $\mathbf{X}$ as shown in Equation 7.3.

$$
\mathbf{Y}=\frac{\mathbf{X}}{\mathbf{Q}}
$$

where $\mathbf{Q}$ divides $\mathbf{X}$ element-by-element.

\subsubsection{Example}

This section purpose is to demonstrate that the method we developed works for our goal to de-quantize $4 \times 4$ blocks of DCT coefficients that were generated from a H.264 forward transform. Let $\mathrm{X}$ be a $4 \times 4$ block of samples from an image as shown in Equation (7.4).

$$
\mathbf{X}=\left[\begin{array}{llll}
158 & 141 & 111 & 80 \\
155 & 141 & 115 & 84 \\
151 & 141 & 120 & 92 \\
152 & 142 & 124 & 98
\end{array}\right]
$$

As shown in Equation 7.5, let $Y$ be the $4 \times 4$ block of coefficients generated from applying the default $\mathrm{H} .264$ forward transform with a quantization parameter, $\mathrm{QP}=6$, on the sample block $\mathbf{X}$.

$$
\mathbf{Y}=\left[\begin{array}{cccc}
401 & 79 & -13 & 9 \\
-8 & 12 & 1 & 0 \\
1 & 1 & 1 & 0 \\
-1 & 0 & 0 & 0
\end{array}\right]
$$

Using Equation (7.1), the quantization matrix $\mathbf{Q}$ is calculated, which is shown in Equation (7.6)

$$
\mathrm{Q}=\left[\begin{array}{llll}
1.2500 & 1.2353 & 1.2500 & 1.2353 \\
1.2353 & 1.2500 & 1.2353 & 1.2500 \\
1.2500 & 1.2353 & 1.2500 & 1.2353 \\
1.2353 & 1.2500 & 1.2353 & 1.2500
\end{array}\right]
$$

Apply quantization matrix $\mathbf{Q}$ on $\mathbf{Y}$ using Equation 7.2 that produced $\mathbf{Z}$.

$$
\mathbf{Z}=\mathbf{Y} \bullet \mathbf{Q}
$$




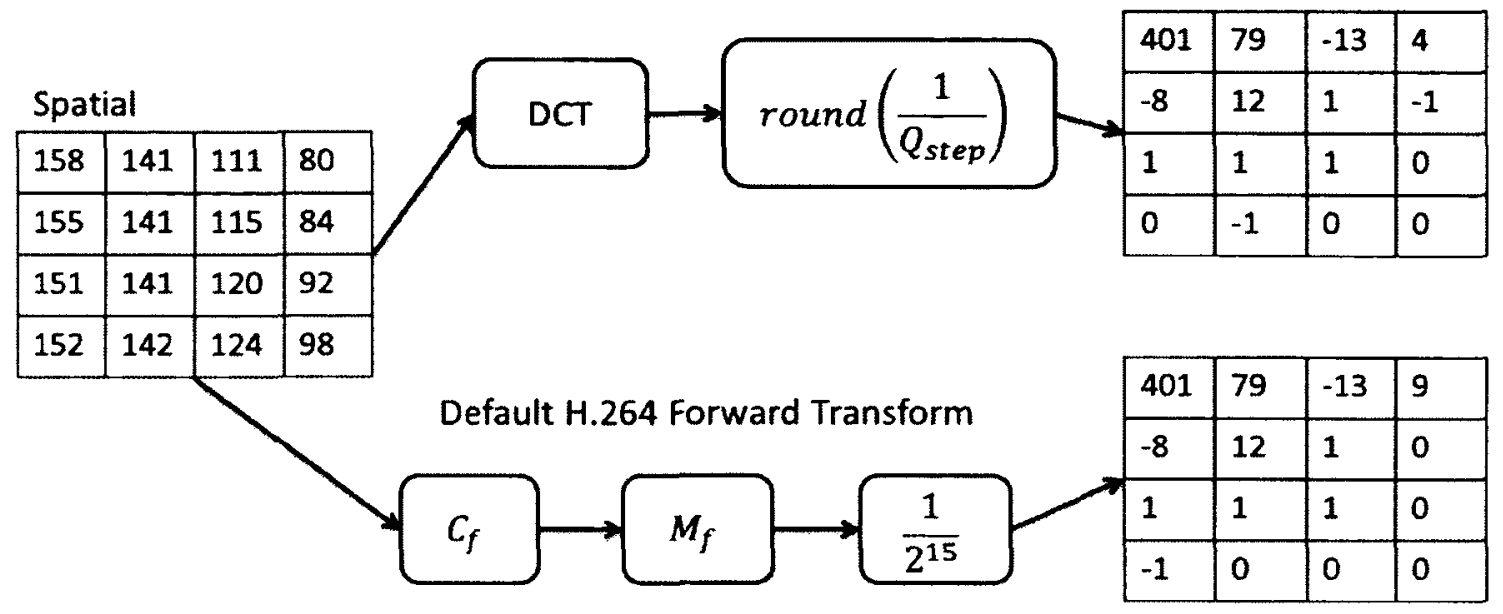

Figure 7.3: The comparing default forward h.264/AVC transform process with regular DCT transforms with quantization.

$$
=\left[\begin{array}{cccc}
501.25 & 97.59 & -16.25 & 11.12 \\
-9.88 & 15 & 1.24 & 0 \\
1.25 & 1.24 & 1.25 & 0 \\
-1.24 & 0 & 0 & 0
\end{array}\right]
$$

The default forward H.264/AVC transform process is similar if an encoder employs a standard DCT type II transform followed by a quantization which divides each coefficient by a $Q_{\text {step }}$ and rounds. In this example, if the $\mathbf{Q P}=6$ then the $Q_{\text {step }}=1.25$. The Figure 7.3 illustrates the two processes transforming pixels from the spatial domain and creating almost identical transform coefficients.

Let $\hat{\mathbf{Y}}$ be coefficients generated from a process that employs DCT type II transform followed by quantization. If we scalar multiply with the constant value $Q_{\text {step }}=1.25$ with $\hat{\mathbf{Y}}$ we will get the scaled coefficients $\hat{\mathbf{Z}}$.

$$
\begin{aligned}
\hat{\mathbf{Z}} & =\hat{\mathbf{Y}} \cdot Q_{\text {step }} \\
& =\left[\begin{array}{cccc}
401 & 79 & -13 & 4 \\
-8 & 12 & 1 & -1 \\
1 & 1 & 1 & 0 \\
0 & -1 & 0 & 0
\end{array}\right] \cdot 1.25
\end{aligned}
$$




$$
=\left[\begin{array}{cccc}
501.25 & 98.75 & -16.25 & 5 \\
-10 & 15 & 1.25 & -1.25 \\
1.25 & 1.25 & 1.25 & 0 \\
0 & -1.25 & 0 & 0
\end{array}\right]
$$

Notice that $\mathbf{Z} \approx \hat{\mathbf{Z}}$. So this example is not proof but illustrates that the our method to dequantize coefficients created from H.264/AVC forward transform process should work.

\subsection{Properties of Conversion Matrix}

This section covers properties that were discovered during our research and were not expressed in the papers by Jiang and Feng [22] or authors that used their work such as Mukherjee and Mitra [21] or Mukhopadhyay [14]. These properties helps us explain why quantization step can be removed from the resizing process.

Chapter 2 introduced the technique of Composition and Decomposition of DCT blocks that was developed by Jiang and Feng [22]. Their work allows algorithms to linearly transform or map coefficients expressed in vector space of $m$ dimensions to other vector space of $n$ dimensions.

To compose a block $N M$ coefficients from $M$ blocks of $N \times N$ coefficients, first a conversion matrix $\mathbf{A}_{(M, N)}$ has be defined as follows:

$$
\mathbf{A}_{(M, N)}=\mathbf{C}_{M N} \cdot\left[\begin{array}{ccc}
\mathbf{C}_{N}^{T} & \cdots & 0_{N} \\
\vdots & \ddots & \vdots \\
0_{N} & \cdots & \mathbf{C}_{N}^{T}
\end{array}\right]
$$

where $\mathbf{C}_{M N}$ is a $M N$-point DCT matrix and $\mathbf{C}_{N}$ is a $N$-point DCT matrix. The submatrix $0_{N}$ are zero matrix of size $N \times N$ and the operator $T$ denotes a transpose operator. In Jiang and Feng paper [22], they used inverse (-1) operator instead of $T$. Since, $C_{N}^{T}=C_{N}^{-1}$ when $I=C_{N} \cdot C_{N}^{T}$, we selected to use the transpose because it is trivial to compute.

Let $\mathbf{B}_{00}, \mathbf{B}_{01}, \mathbf{B}_{10}$ and $\mathbf{B}_{11}$ be four adjacent blocks of size $N \times N$ and their coefficients are a linear combination of a $N$-point DCT. If we want to compose these blocks to create one block $\mathrm{B}$ of size $N M \times N M$ so the coefficients are a linear 
combination of a $M N$-point DCT we do the following:

$$
\mathbf{B}=\mathbf{A}_{(M, N)} \cdot\left[\begin{array}{ll}
\mathbf{B}_{00} & \mathbf{B}_{01} \\
\mathbf{B}_{10} & \mathbf{B}_{11}
\end{array}\right] \cdot \mathbf{A}_{(M, N)}^{T}
$$

where $T$ denotes the transpose operator. To decompose $\mathbf{B}$ back to $M$ blocks of size $N \times N$ where coefficients are linear combination of $N$-point DCT do the following.

$$
\left[\begin{array}{ll}
\mathbf{B}_{00} & \mathbf{B}_{01} \\
\mathbf{B}_{10} & \mathbf{B}_{11}
\end{array}\right]=\mathbf{A}_{(M, N)}^{T} \cdot \mathbf{B} \cdot \mathbf{A}_{(M, N)}
$$

So the conversion matrix $\mathbf{A}_{(M, N)}$ provides an invertible linear transformation of coefficients between two vector spaces with different dimensions.

This fact leads us to believe that the conversion matrix $\mathbf{A}_{(M, N)}$ was orthonormal, which means the rows of $\mathbf{A}_{(M, N)}$ are of unit norm and the row vectors are orthogonal to each other (i.e. inner product is zero). From experiments, we discovered that $\mathbf{A}_{(M, N)}$ was orthonormal which implied the following:

$$
\mathbf{I}=\mathbf{A}_{(M, N)} \cdot \mathbf{A}_{(M, N)}^{T}
$$

, where the $\mathbf{I}$ is the identity matrix.

Orthonormal transforms are energy preserving. This means that the sum of the square of the transformed sequence is equal to the sum of the squares of the original sequence. Sayood's book [17] illustrates a proof this. Let $\left\{x_{n}\right\}$ represent the original sequence and $\left\{\theta_{n}\right\}$ the transformed sequence. We can see that orthonormal transforms are energy preserving in the case of the one-dimensional transform.

$$
\begin{aligned}
\sum_{l=0}^{N-1} \theta_{l}^{2} & =\theta^{T} \theta \\
& =(\mathbf{A} \mathbf{x})^{T} \mathbf{A} \mathbf{x} \\
& =\mathbf{x}^{T} \mathbf{A}^{T} \mathbf{A} \mathbf{x}
\end{aligned}
$$

If $\mathbf{A}$ is an orthonormal transform, $\mathbf{A}^{T} \mathbf{A}=\mathbf{A}^{-1} \mathbf{A}=\mathbf{I}$, then

$$
\begin{aligned}
\mathbf{x}^{T} \mathbf{A}^{T} \mathbf{A} \mathbf{x} & =\mathbf{x}^{T} \mathbf{x} \\
& =\sum_{n=0}^{N-1} x_{n}^{2}
\end{aligned}
$$


and

$$
\sum_{i=0}^{N-1} \theta_{i}^{2}=\sum_{n=0}^{N-1} x_{n}^{2} .
$$

Quantization can be expressed as scalar multiplication by a constant followed by rounding. After quantization has been applied on the blocks of DCT coefficients, reconstructing the exact image is impossible because of this rounding. Also, quantization and rounding will make some or most of the DCT coefficients to become zero depending on the quantization step. What is left are a few low frequency coefficients where most of the signal energy is stored. Resizing algorithms that compose and decompose DCT blocks by applying conversion matrices $\mathbf{A}_{(M, N)}$ preserve the energy of the original image in the resized image. Therefore, applying forward quantization before DCT resizing is unnecessary. Only decoder will required to apply inverse quantization before transforming the DCT coefficients back into the spatial domain.

\subsection{Experimental Setup}

This section describes the experimental setup used to evaluate two DCT domain resizing process as illustrated in Figure 7.1 and 7.2. The experiment is based on a similar approach used in Chapter 6 when measuring image quality. In both processes, images in the sample set outlined in Appendix D are first halved then doubled, which is carry out as follows. First, our H.264/AVC encoder compressed the original image. Then the halving application read in the compressed image and outputted a compressed halved image. This image is then read in by the doubling application and outputted a compressed image in the original resolution. Our H.264/AVC decoder transformed the doubled image back into the spatial domain. Then we computed the PSNR value by comparing resultant doubled image with the original image.

For both resizing processes, we applied the IHAC algorithm for halving the image followed by IDDA algorithm for doubling. We choose these algorithms because they represented a class of algorithms that employed conversion matrices to implement resizing, such as IHCA, IDAD, LMUS, LMDS, and HRA. Since the conversion matrices in these resizing algorithms are orthonormal, we extrapolated that the new process will provide similar results. However, we need to prove this hypothesis through experimentation. 
After the image is doubled, entropy encoding is applied to the resultant image to obtain the bitrate measurement. We used a Context Adaptive Variable Length Coding (CAVLC) entropy encoder to calculate the bitrate of the doubled image for both processes.

\subsection{Presentation of Experimental Results}

This section outlines the experimental results of the two DCT domain resizing process. We applied the experiment multiple times over different QP levels. Table 7.1 shows the mean image quality of the two processes over multiple QP levels. Table 7.2 displays the mean bitrate for both processes over multiple QP levels. Also, this table outlines the bitrate saving if resizing process includes quantization, which is calculated as follows:

$$
\text { bitrate savings }=1-\frac{\text { bitrate of process including } \mathbf{Q}}{\text { bitrate of process excluding } \mathbf{Q}}
$$

So bitrate savings expresses the percentage of the bitrate reduction from a process that includes quantization over a process without.

Table 7.1: The mean PSNR value with standard deviation $\sigma$ when comparing DCT domain resizing processes that includes quantization and one without quantization over multiple QP levels.

\begin{tabular}{c|c|c||c|c}
\hline \hline QP & $\begin{array}{c}\text { Without } \\
\text { Quantization }\end{array}$ & $\sigma$ & $\begin{array}{c}\text { With } \\
\text { Quantization }\end{array}$ & $\sigma$ \\
\hline \hline 6 & 31.34 & 3.81 & 31.34 & 3.81 \\
12 & 31.26 & 3.74 & 31.26 & 3.74 \\
18 & 31.01 & 3.53 & 31.01 & 3.53 \\
24 & 30.33 & 3.11 & 30.34 & 3.12 \\
30 & 28.99 & 2.66 & 28.99 & 2.66 \\
36 & 26.90 & 2.27 & 26.91 & 2.27 \\
42 & 24.53 & 1.99 & 24.64 & 2.00 \\
48 & 21.89 & 1.51 & 21.95 & 1.51 \\
\hline \hline
\end{tabular}


Table 7.2: The mean bitrate with standard deviation $\sigma$ when comparing DCT domain resizing processes that includes quantization and one without quantization over multiple QP levels. The last column outlines the bitrate saving if process includes quantization.

\begin{tabular}{c|c|c||c|c||c}
\hline \hline QP & $\begin{array}{c}\text { Without } \\
\text { (Kiantization } \\
\text { (Kilobits) }\end{array}$ & $\begin{array}{c}\sigma \\
\text { (Kilobits) }\end{array}$ & $\begin{array}{c}\text { Wuanth } \\
\text { (Kilobits) }\end{array}$ & $\begin{array}{c}\sigma \\
\text { (Kilobits) }\end{array}$ & $\begin{array}{c}\text { Bitrate } \\
\text { Savings }\end{array}$ \\
\hline \hline 6 & $1,047.07$ & 112.58 & $1,045.60$ & 113.04 & $0.14 \%$ \\
12 & 885.96 & 82.18 & 885.42 & 82.28 & $0.06 \%$ \\
18 & 763.39 & 57.68 & 759.62 & 58.48 & $0.49 \%$ \\
24 & 667.45 & 43.64 & 663.15 & 44.15 & $0.64 \%$ \\
30 & 578.89 & 45.05 & 578.77 & 45.01 & $0.02 \%$ \\
36 & 445.61 & 54.12 & 445.57 & 54.08 & $0.01 \%$ \\
42 & 294.74 & 49.49 & 291.02 & 50.17 & $1.26 \%$ \\
48 & 166.06 & 20.52 & 163.91 & 20.12 & $1.29 \%$ \\
\hline \hline
\end{tabular}

\subsection{Analysis of Results}

The goal of this research was to determine if quantization step can be removed from DCT domain resizing process to create a new process as show in Figure 7.2. Two performance measurements were used to compare the two processes: 1) image quality and 2) bitrate.

The size of an uncompressed image file with a resolution of $512 \times 512$ is 256 Kilobtyes or 2,048 Kilobits. When comparing the uncompressed size with values outlined in Table 7.2, it shows there are compression savings using H.264/AVC transform process with CAVLC entropy encoding, especially at higher QP levels. This confirmed to us that both processes were working as expected and producing bitrate well below the uncompressed file size.

Both resizing processes produced images with similar mean PSNR values. However, the bitrate savings for the resizing process that included quantization was approximately $1 \%$ or less. Therefore, the bitrate saving was negligible for the incurred computational cost. There was increase bitrate savings when QP $>36$ of almost $1 \%$. This deviation maybe caused by rounding, which is part of the quantization process. At higher QP levels, more coefficients will be rounded down to zero, which lowers bitrate. 
The computational cost of including quantization into the resizing process is as follows. Let $M$ be the number of multiplications. A $4 \times 4$ DCT block contains 16 coefficients. There are 16 entries in the quantization matrix $\mathbf{Q}$. The process that includes quantization applies the matrix $\mathbf{Q}$ twice; once before resizing (inverse quantization) and once after resizing (forward quantization with rounding). Let IHAC algorithm be used for a resizing process. The IHAC algorithm inputs a $2 \times 2$ block containing four adjacent $4 \times 4$ blocks of coefficients. Applying inverse quantization would cost $64 M$ because the process multiplies element-by-element $\mathbf{Q}$ four times over four $4 \times 4$ DCT blocks of coefficients. After resizing; the forward quantization is applied on one $4 \times 4$ DCT block, which will cost $16 M$. Therefore, the total cost is $1.25 M=(64 M+16 M) / 64$ per pixel of the original image. 


\section{Chapter 8}

\section{Efficient Resizing Algorithms in the DCT Domain}

\subsection{Introduction}

Image halving is an operation that takes an image of size $N \times N$ and outputs an image of $N / 2 \times N / 2$, where image doubling is the inverse operation to resize an image to a size of $2 N \times 2 N$. Let $b$ an $N \times N$ block in the spatial domain whose DCT coefficients are encoded as $N \times N$ block $\mathbf{B}$ in the compressed domain, with elements $B(k, l), k, l=0,1,2 \ldots 7$. Generally, to halve an image one needs to convert four adjacent DCT blocks, $\mathbf{B}_{1}, \mathbf{B}_{2}, \mathbf{B}_{3}$ and $\mathbf{B}_{4}$, to a single $N \times N$ DCT block, $\hat{\mathbf{B}}$. In Mukherjee and Mitra [13] IHAC algorithm, four $\frac{N}{2} \times \frac{N}{2}$ adjacent blocks are derived from the corresponding $N \times N$ DCT blocks using subband approximation. Then the decimated $\frac{N}{2} \times \frac{N}{2}$ blocks are recomposed to form an single $N \times N$ block using an conversion matrix in the block composition step. Figure 8.1 provides an illustration of this algorithm.

To double an image Mukherjee and Mitra [13] employed DCT block decomposition. In the IDDA algorithm, an $N \times N$ DCT block is first decomposed using conversion matrix to four $\frac{N}{2} \times \frac{N}{2}$ DCT blocks. Then each of these blocks are transformed into an $N \times N$ DCT block by using subband approximation and zero-padding.

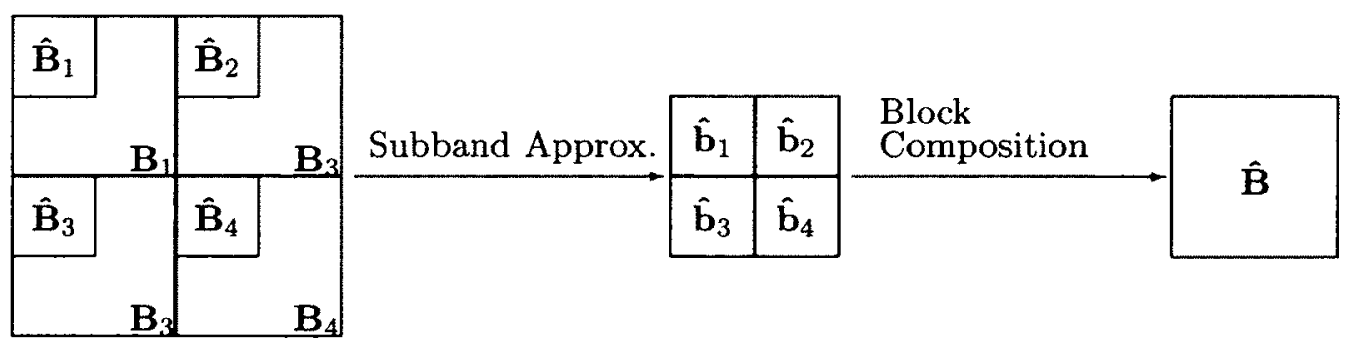

Figure 8.1: IHAC [13]. Four $\frac{N}{2} \times \frac{N}{2}$ approximated DCT coefficients of adjacent blocks are composed into one $N \times N$ DCT block. 


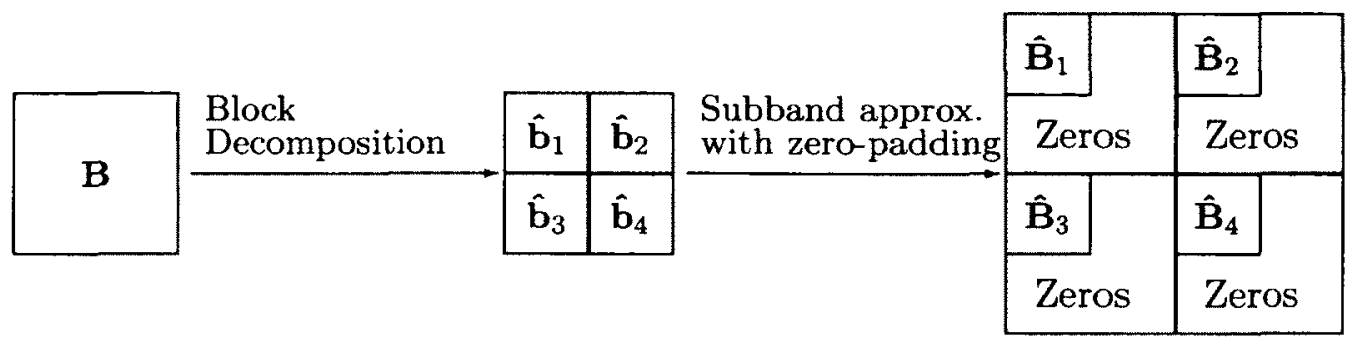

Figure 8.2: IDDA [13]. An $N \times N$ DCT block is decomposed into four $\frac{N}{2} \times \frac{N}{2}$ blocks where each is approximated to an $N \times N$ DCT block with zero-padding.

Figure 8.2 presents a schematic representation of this algorithm.

We propose to enhance these two algorithms by deriving a fix-point approximation of the conversion matrices used in the block composition and decomposition step. We followed a similar approach that the H.264/AVC standard body developed to define the default inverse transform process. Malvar et al [3] [19] reported a reduction in complexity with negligible impact on image quality when using a fix-point approximation of a $4 \times 4$ DCT matrix.

H.264/AVC compressed image is based on a $4 \times 4$ DCT block framework, hence $N=4$. Therefore, an IHAC resize algorithm to resize $\mathrm{H} .264 /$ AVC frame would contain the following composition step.

$$
\begin{aligned}
\mathbf{B}_{d} & =\mathbf{A}_{(2,2)}\left[\begin{array}{ll}
\hat{\mathbf{B}}_{00}^{(2 \times 2)} & \hat{\mathbf{B}}_{01}^{(2 \times 2)} \\
\hat{\mathbf{B}}_{10}^{(2 \times 2)} & \hat{\mathbf{B}}_{11}^{(2 \times 2)}
\end{array}\right] \mathbf{A}_{(2,2)}^{T} \\
\mathbf{B}_{d} & =\mathbf{A}_{(2,2)} \cdot \hat{\mathbf{B}} \cdot \mathbf{A}_{(2,2)}^{T}
\end{aligned}
$$

where $\cdot$ denotes matrix multiplication and the conversion matrix:

$$
\mathbf{A}_{(2,2)}=\left[\begin{array}{cccc}
1 / \sqrt{2} & 0 & 1 / \sqrt{2} & 0 \\
0.6533 & 0.2706 & -0.6533 & 0.2706 \\
0 & 1 / \sqrt{2} & 0 & -1 / \sqrt{2} \\
-0.2706 & 0.6533 & 0.2706 & 0.6533
\end{array}\right]
$$

Note that the rows of $\mathbf{A}_{(2,2)}$ are orthogonal and unit norm, which is a necessary condition for an orthogonal block transform. All the entries in $\mathbf{A}_{(2,2)}$ require processors to approximate irrational numbers. A fixed-point approximation is equivalent to 
scaling each row of $\mathbf{A}_{(2,2)}$ and rounding to the nearest integer. We chose the same number that the H.264/AVC standard body selected and multiplied $\mathbf{A}_{(2,2)}$ by 2.5 and rounded, which gives us $\mathbf{C}_{(\mathbf{2}, \mathbf{2})}$ :

$$
\begin{aligned}
\mathbf{C}_{(2,2)} & =\operatorname{round}\left(2.5 \cdot \mathbf{A}_{(2,2)}\right) \\
\mathbf{C}_{(2,2)} & =\left[\begin{array}{cccc}
2 & 0 & 2 & 0 \\
2 & 1 & -2 & 1 \\
0 & 2 & 0 & -2 \\
-1 & 2 & 1 & 2
\end{array}\right]
\end{aligned}
$$

The row norms of $\mathbf{C}_{(\mathbf{2 , 2})}$ are $\neq 1$. To restore the orthonormal property of the original matrix of $\mathbf{A}_{(2,2)}$, we multiply all the values of $c_{i j}$ in row $r$ by $\frac{1}{\sqrt{\sum_{j} c_{r j}^{2}}}$ :

$$
\mathbf{A}_{(2,2)}=\mathbf{C}_{(2,2)} \bullet \mathbf{R}_{(2,2)}
$$

where

$$
\mathbf{R}_{(2,2)}=\left[\begin{array}{cccc}
1 / \sqrt{8} & 1 / \sqrt{8} & 1 / \sqrt{8} & 1 / \sqrt{8} \\
1 / \sqrt{10} & 1 / \sqrt{10} & 1 / \sqrt{10} & 1 / \sqrt{10} \\
1 / \sqrt{8} & 1 / \sqrt{8} & 1 / \sqrt{8} & 1 / \sqrt{8} \\
1 / \sqrt{10} & 1 / \sqrt{10} & 1 / \sqrt{10} & 1 / \sqrt{10}
\end{array}\right]
$$

The operator $\bullet$ denotes an element-by-element multiplication. The two-dimensional transform in Equation 8.1 becomes:

$$
\mathbf{B}_{d}=\mathbf{A}_{(2,2)} \cdot \hat{\mathbf{B}} \cdot \mathbf{A}_{(2,2)}^{T}=\left[\mathbf{C}_{(2,2)} \bullet \mathbf{R}_{(2,2)}\right] \cdot \hat{\mathbf{B}} \cdot\left[\mathbf{C}_{(2,2)}^{T} \bullet \mathbf{R}_{(2,2)}^{T}\right]
$$

Rearranging to extract the scaling arrays $\mathbf{R}_{(2,2)}$ :

$$
\begin{aligned}
\mathbf{B}_{d} & =\left[\mathbf{C}_{(2,2)} \cdot \hat{\mathbf{B}} \cdot \mathbf{C}_{(2,2)}^{T}\right] \bullet\left[\mathbf{R}_{(2,2)} \bullet \mathbf{R}_{(2,2)}^{T}\right] \\
& =\left[\mathbf{C}_{(2,2)} \cdot \hat{\mathbf{B}} \cdot \mathbf{C}_{(2,2)}^{T}\right] \bullet \mathbf{S}_{(2,2)}
\end{aligned}
$$

where

$$
\mathbf{S}_{(2,2)}=\mathbf{R}_{(2,2)} \bullet \mathbf{R}_{(2,2)}^{T}=\left[\begin{array}{cccc}
1 / 8 & 1 / \sqrt{80} & 1 / 8 & 1 / \sqrt{80} \\
1 / \sqrt{80} & 1 / 10 & 1 / \sqrt{80} & 1 / 10 \\
1 / 8 & 1 / \sqrt{80} & 1 / 8 & 1 / \sqrt{80} \\
1 / \sqrt{80} & 1 / 10 & 1 / \sqrt{80} & 1 / 10
\end{array}\right]
$$


Using the new fixed-point conversion matrix $\mathbf{C}_{(2,2)}$ and its scaling matrix $\mathbf{S}_{(2,2)}$, we modified the IHAC algorithm. Algorithm 14 outlines our new proposed image halving algorithm that takes advantage of these matrices in the composition step.

Algorithm 14 Integer Based IHAC for H.264/AVC Frames

Input: $4 \times 4$ block H.264 based DCT encoded image.

Output: $4 \times 4$ block based DCT encoded downsampled image.

for every four adjacent $4 \times 4, \mathbf{B}_{00}, \mathbf{B}_{01}, \mathbf{B}_{10}, \mathbf{B}_{11}$ of the input image do

1. Subband approximation: Get corresponding $2 \times 2$ 2-point DCT blocks $\left\{\hat{\mathbf{B}}_{i j}^{(2 \times 2)}\right\}, i=0,1$ and $j=0,1$ using 2.29 , low-pass truncated approximation, as follows.

$$
\hat{\mathbf{B}}_{i j}^{(2 \times 2)}=\frac{1}{2}\left[\mathbf{B}_{i j}(k, l)\right]_{0 \leq k, l \leq 1}
$$

2. Block composition: Convert four $2 \times 2$ DCT blocks to a $4 \times 4$ DCT block $\mathbf{B}_{d}$ as follows.

$$
\mathbf{B}_{d}=\left(\mathbf{C}_{(2,2)}\left[\begin{array}{cc}
\hat{\mathbf{B}}_{00}^{(2 \times 2)} & \hat{\mathbf{B}}_{01}^{(2 \times 2)} \\
\hat{\mathbf{B}}_{10}^{(2 \times 2)} & \hat{\mathbf{B}}_{11}^{(2 \times 2)}
\end{array}\right] \mathbf{C}_{(2,2)}^{T}\right) \bullet \mathbf{S}_{(2,2)}
$$

end for

Similarly, we modified the IDDA algorithm in the decomposition step to leverage the fixed-point approximation of the conversation matrix with its scaling matrix. Algorithm 15 displays the outline of our proposed integer based IDDA algorithm. 
Algorithm 15 Integer Based IDDA for H.264/AVC Frames

Input: $4 \times 4$ block based DCT encoded image.

Output: Upsampled image in the compressed domain.

for each $4 \times 4$ blocks $\mathbf{B}$ do the following: do

1. Block decomposition Convert the block to four $2 \times 2$ DCT blocks as follows.

$$
\left[\begin{array}{ll}
\mathbf{B}_{00}^{(2 \times 2)} & \mathbf{B}_{01}^{(2 \times 2)} \\
\mathbf{B}_{10}^{(2 \times 2)} & \mathbf{B}_{11}^{(2 \times 2)}
\end{array}\right]=\left(\mathbf{C}_{(2,2)}^{T} \mathbf{B C}_{(2,2)}\right) \bullet \mathbf{S}_{(2,2)}
$$

2. Subband approximation and zero padding Compute the approximate $4 \times 4$-point DCT coefficients from each of $\mathbf{B}_{i j}^{(2 \times 2)}, i=0,1$ and $j=0,1$ from 2.29 , low-pass truncated approximation. Form four $4 \times 4$ DCT blocks by zero padding each of them (the high frequency components are assigned to zero $0_{2}$ ).

$$
\mathbf{B}_{i j}^{(4 \times 4)}=2\left[\begin{array}{cc}
\mathbf{B}_{i j}^{(2 \times 2)} & 0_{2} \\
0_{2} & 0_{2}
\end{array}\right]
$$

end for

\subsection{Experimental Results}

We developed three experiments to evaluate image quality performance of our proposed Integer based IHAC (IIHAC) 14 and Integer based IDDA (IIDDA) 15 algorithms. In the first experiment, for each image in the sample set $I_{\text {orig }}$ of size $N \times N$ is first spatially downsampled using MATLAB bicubic interpolation with anti-aliasing to an image $I_{d}$ of size $N / 2 \times N / 2$. This provided a reference image so PSNR can be computed when comparing images produced from halving algorithms. We compared the PSNR values computed from images generated from IIHAC and its floating-point based IHAC version as described in Algorithm 8.

For the second experiment to evaluate image quality performance of the IIDDA algorithms, spatially downsampled images $I_{d}^{s}$ are compressed then upsampled in the compressed domain. We used MATLAB bicubic interpolation with anti-aliasing to downsample images $I_{d}^{s}$ in the spatial domain. After doubling, PSNR values are computed between the upsampled images with the original images $I_{\text {orig }}$. We compared 
the PSNR values computed from images generated from IIDDA and its floating-point base IDDA version as describe in Algorithm 10.

In the third experiment, an image is first halved and then doubled in the compressed domain. The resulting upsampled image is compared with the original image $I_{\text {orig. }}$. We compared the PSNR valued computed from images generated from resizing algorithms used in tandem: IHS-IDS, IHAC-IDDA and IIHAC-IIDDA. The IHS first halved using bilinear interpolation without anti-aliasing while the IDS doubles the image using zero-order interpolation. All these experiments are presented in Table 8.1 .

\subsection{Computational Cost}

This section outlines the computational cost of our proposed Integer based resizing algorithm. By using an integer transform in the proposed algorithms, we hope to increase computational efficiency. Leveraging fixed-point arithmetic, multiplying or dividing values that are a power of two can be accomplished by binary shift operations. You will observe that the conversion matrix $\mathbf{C}_{(2,2)}$ entries are 0, 1's or 2's. Therefore, matrix multiplications can be carried out multiplier-free. A matrix is multiplier-free if the matrix multiplication can be carry-out using only additions and shifts operations.

Let $n_{m}$ be number of multiplications and $n_{a}$ be number of additions. In Table 8.2 , outlines computational cost for the IIHAC algorithm. Table 8.3 outlines the computational cost for the IIDDA algorithm. Also, the tables outlined quality/cost ratio where the larger the number indicates greater the efficiency in creating a number of dB's.

The Table 8.4 shows how IIHAC compares with other image halving algorithms, while Table 8.5 shows how IIDDA compares with image doubling algorithms. In all cases, the integer based algorithms was the most computational efficient. The computational savings is calculated as follows:

$$
\text { computational savings }=1-\frac{n_{o p} \text { of integer based algorithm }}{n_{o p} \text { of non-integer based algorithm }}
$$

where $n_{o p}$ denotes either the number of additions $n_{a}$ or multiplications $n_{m}$.

When comparing IIHAC algorithm with its floating-point implementation, it was $63 \%$ more efficient in $n_{m}$ and $75 \%$ in $n_{a}$. As for IIDDA, it was $91 \%$ more efficient in 
Table 8.1: Results from three experiments used to evaluate image quality performance of IIHAC and IIDDA resize algorithms.

\begin{tabular}{c||c|c||c|c||c|c|c}
\hline \hline \multicolumn{1}{c||}{ Images } & \multicolumn{2}{c||}{ Experiment 1 } & \multicolumn{2}{|c||}{ Experiment 2 } & \multicolumn{3}{c}{ Experiment 3} \\
\cline { 2 - 7 } & IHAC & IIHAC & IDDA & IIDDA & IHS & IHAC & IIHAC \\
& & & & & IDS & IDDA & IIDDA \\
\hline \hline aerial_buildings.raw & 39.05 & 36.54 & 26.6 & 26.2 & 25.66 & 26.85 & 26.55 \\
apc.raw & 45.72 & 44.31 & 33.87 & 33.66 & 33.52 & 34.12 & 33.96 \\
apc_two_vehs1.raw & 42.10 & 40.28 & 29.87 & 29.59 & 29.35 & 30.11 & 29.91 \\
apc_two_vehs2.raw & 41.93 & 40.14 & 29.67 & 29.41 & 29.17 & 29.92 & 29.72 \\
apc_two_vehs3.raw & 45.89 & 43.78 & 34.35 & 33.99 & 33.51 & 34.61 & 34.33 \\
Barbara.raw & 37.8 & 36.88 & 25.48 & 25.37 & 25.63 & 25.73 & 25.65 \\
boat.raw & 41.28 & 39.08 & 29.34 & 28.97 & 28.55 & 29.61 & 29.34 \\
cameraman.raw & 44.46 & 40.62 & 33.47 & 32.67 & 31.09 & 33.77 & 33.08 \\
Elaine.raw & 45.31 & 42.53 & 32.65 & 32.33 & 31.74 & 32.86 & 32.54 \\
Goldhill.raw & 42.79 & 40.77 & 30.97 & 30.64 & 30.35 & 31.24 & 31.00 \\
house.raw & 49.25 & 44.07 & 40.42 & 38.77 & 35.91 & 40.84 & 39.15 \\
jetplane.raw & 42.76 & 39.69 & 30.45 & 29.96 & 28.86 & 30.69 & 30.30 \\
lake.raw & 41.89 & 38.7 & 29.65 & 29.13 & 28.28 & 29.89 & 29.48 \\
landed_jet.raw & 46.9 & 44.64 & 35.31 & 34.91 & 34.28 & 35.55 & 35.22 \\
Lena.raw & 44.62 & 41.43 & 33.03 & 32.46 & 31.52 & 33.30 & 32.82 \\
livingroom.raw & 41.20 & 39.16 & 28.9 & 28.58 & 28.28 & 29.15 & 28.93 \\
mandril.raw & 36.13 & 35.08 & 23.48 & 23.33 & 23.35 & 23.72 & 23.63 \\
peppers.raw & 43.87 & 40.83 & 31.44 & 31.07 & 29.96 & 31.67 & 31.31 \\
pirate.raw & 42.49 & 40.07 & 30.37 & 29.99 & 29.52 & 30.62 & 30.33 \\
tank_front.raw & 42.82 & 41.37 & 30.64 & 30.42 & 30.28 & 30.89 & 30.74 \\
tank_side.raw & 42.96 & 41.30 & 30.83 & 30.58 & 30.40 & 31.08 & 30.9 \\
tank_top.raw & 44.62 & 43.12 & 32.49 & 32.26 & 32.09 & 32.72 & 32.56 \\
truck.raw & 44.68 & 42.68 & 32.87 & 32.54 & 32.20 & 33.13 & 32.88 \\
walkbridge.raw & 39.07 & 37.07 & 26.67 & 26.38 & 26.14 & 26.92 & 26.71 \\
watch.raw & 40.51 & 36.99 & 27.47 & 27.01 & 25.98 & 27.69 & 27.31 \\
woman_blonde.raw & 41.94 & 40.06 & 29.28 & 29.04 & 28.57 & 29.51 & 29.32 \\
woman_darkhair.raw & 49.35 & 45.81 & 39.64 & 38.89 & 37.15 & 39.94 & 39.14 \\
\hline Mean & 43.01 & 40.63 & 31.08 & 30.67 & 30.05 & 31.34 & 30.99 \\
\hline \hline & & & & & & & \\
\hline
\end{tabular}


Table 8.2: IIHAC Account of Computations

\begin{tabular}{c|c|c|l}
\hline \hline Equations & $n_{m}$ & $n_{a}$ & Note \\
\hline$\frac{1}{2}\left[\mathbf{B}_{i j}(k, l)\right]_{0 \leq k, l \leq 1}$ & 0 & 0 & $\begin{array}{l}\text { Multiplying by a half can be } \\
\text { done by on right shift opera- } \\
\text { tion. }\end{array}$ \\
\hline $\mathbf{B}_{t}=\left(\mathbf{C}_{(2,2)}\left[\begin{array}{ll}\hat{\mathbf{B}}_{00}^{(2 \times 2)} \\
\hat{\mathbf{B}}_{10}^{(2 \times 2)} & \hat{\mathbf{B}}_{01}^{(2 \times 2)} \\
\hat{\mathbf{B}}_{11}^{(2 \times 2)}\end{array}\right] \mathbf{C}_{(2,2)}^{T}\right)$ & 0 & 16 & $\begin{array}{l}\text { No multiplications because } \\
\text { the entries in } \mathbf{C}_{(2,2)} \text { are either } \\
0,1,-1,2 \text { or -2. Only addi- } \\
\text { tions. }\end{array}$ \\
\hline $\mathbf{B}_{d}=\mathbf{B}_{t} \bullet \mathbf{S}_{(2,2)}$ & 12 & 0 & $\begin{array}{l}\text { This is element-by-element } \\
\text { multiplication. The entries } \\
\text { in } \mathbf{S}(2,2) \text { equal to 1/8 can be } \\
\text { done using a right shift oper- } \\
\text { ation. }\end{array}$ \\
\hline \hline Total & 12 & 16 & \\
\hline \hline
\end{tabular}

Table 8.3: IIDDA Account of Computations

\begin{tabular}{c|c|c|l}
\hline \hline Equations & $n_{m}$ & $n_{a}$ & Note \\
\hline \hline $\mathbf{B}_{t}=\mathbf{C}_{(2,2)}^{T} \mathbf{B C}_{(2,2)}$ & 0 & 16 & $\begin{array}{l}\text { No multiplications because } \\
\text { the entries in } \mathbf{C}_{(2,2)} \text { are either } \\
0,1,-1,2 \text { or }-2 \text {. Only addi- } \\
\text { tions. }\end{array}$ \\
\hline $\mathbf{B}_{\mathbf{t}} \bullet \mathbf{S}_{(2,2)}$ & 12 & 0 & $\begin{array}{l}\text { This is element-by-element } \\
\text { multiplication. The entries } \\
\text { in } \mathbf{S}_{(2,2)} \text { equal to 1/8 can be } \\
\text { done using a right shift oper- } \\
\text { ation. }\end{array}$ \\
\hline \hline $2\left[\begin{array}{cc|c|c|}\mathbf{B}_{i j}^{(2 \times 2)} & 0_{2} \\
0_{2} & 0_{2}\end{array}\right]$ & 0 & 0 & $\begin{array}{l}\text { The scalar multiplication can } \\
\text { be carried out by a left shift } \\
\text { operation }\end{array}$ \\
\hline Ops per pixel of the upsampled image & 0.1875 & 0.25 & $\begin{array}{l}\text { Four adjacent 4 } 4 \text { 4 DCT } \\
\text { blocks are outputted for a to- } \\
\text { tal of } 64 \text { pixels }\end{array}$ \\
\hline \hline
\end{tabular}


Table 8.4: Comparing computation cost Integer based IHAC (IIHAC) with other image halving algorithms by the number of multiplication $\left(n_{m}\right)$ and additions $\left(n_{a}\right)$ per pixel of the original image

\begin{tabular}{c|c|c|c|c|c|c}
\hline \hline & IHS & IHBI & IHFDS & IHCA & IHAC & IIHAC \\
\hline \hline mean(PSNR) & 43.5 & 41.36 & 43.1 & 40.62 & 43.01 & 40.63 \\
\hline$n_{m}$ & 1.25 & 4.5 & 2 & 4 & 0.5 & 0.1875 \\
\hline$n_{a}$ & 8.25 & 2.5 & 1 & 8 & 1 & 0.25 \\
\hline \hline $\begin{array}{c}\text { quality/cost } \\
\text { mean }\left(\text { PSNR } /\left(n_{m}+n_{a}\right)\right.\end{array}$ & 4.58 & 5.91 & 14.37 & 3.39 & 28.67 & 92.87 \\
\hline \hline
\end{tabular}

Table 8.5: Comparing computation cost Integer based IDDA (IIDDA) with other image doubling algorithms by the number of multiplication $\left(n_{m}\right)$ and additions $\left(n_{a}\right)$ per pixel of the upsampled image

\begin{tabular}{c|c|c|c|c|c|c}
\hline \hline ops & Spatial & IDZOI & IDUSZP & IDAD & IDDA & IIDDA \\
\hline \hline mean(PSNR) & 29.89 & 29.42 & 31.08 & 31.62 & 31.08 & 30.67 \\
\hline$n_{m}$ & 1.25 & 4.5 & 2 & 10 & 2 & 0.1875 \\
\hline$n_{a}$ & 8.25 & 2.5 & 1 & 8 & 1.5 & 0.25 \\
\hline \hline $\begin{array}{c}\text { quality/cost } \\
\text { mean }(P S N R) /\left(n_{m}+n_{a}\right)\end{array}$ & 3.15 & 4.53 & 10.36 & 1.76 & 8.88 & 70.10 \\
\hline \hline
\end{tabular}

$n_{m}$ and $83 \%$ in $n_{a}$ when comparing with its floating-point version.

\subsection{Analysis of Results}

When comparing image quality, our proposed integer based algorithms produced lower PSNR values compared to the floating-point implementations, which was expected because of rounding errors associated with integer transforms and scaling. For the first experiment, the IIHAC version was $2.33 \mathrm{~dB}$ lower than the IHAC but the average PSNR for both algorithms was over $40 \mathrm{~dB}$. From subjective observation, it was hard to see any significant difference between images produces from these two algorithms. Figure 8.3 displays images generate from IIHAC, IHAC and halving in the spatial domain using the IHS algorithm.

For image doubling experiment, the IIDDA algorithm had PSNR value $0.39 \mathrm{~dB}$ lower on average than floating-point version IDDA. When using our proposed algorithms in tandem in the image halving followed by doubling experiment, the PSNR value was $0.35 \mathrm{~dB}$ lower than IHAC-IDDA but $0.94 \mathrm{~dB}$ better than image resizing in 
the spatial domain using IHS-IDS pairing.

When comparing computational cost with our proposed efficient algorithms with image resizing in spatial domain, it is a significant savings for applications using our integer based algorithms. For quality/cost ratio, our proposed algorithm was very efficient in producing high number of dB's for very little computational cost. Our proposed algorithm was $69 \%$ more efficient for image halving and $62 \%$ more efficient for image doubling than the comparable floating-point versions.

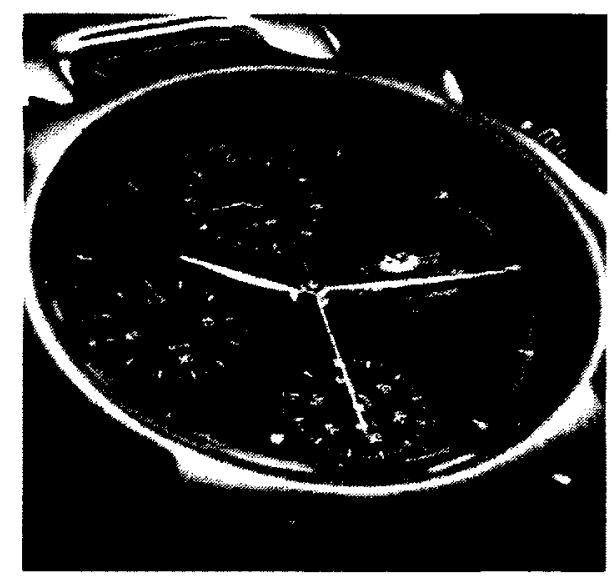

(a) IHS

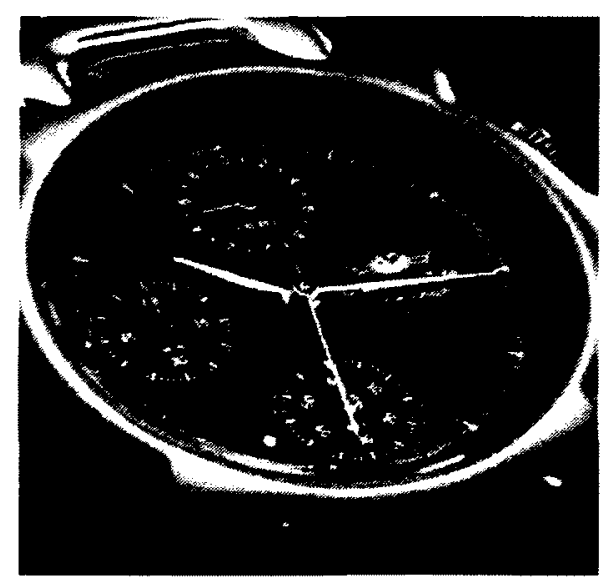

(b) IIHAC

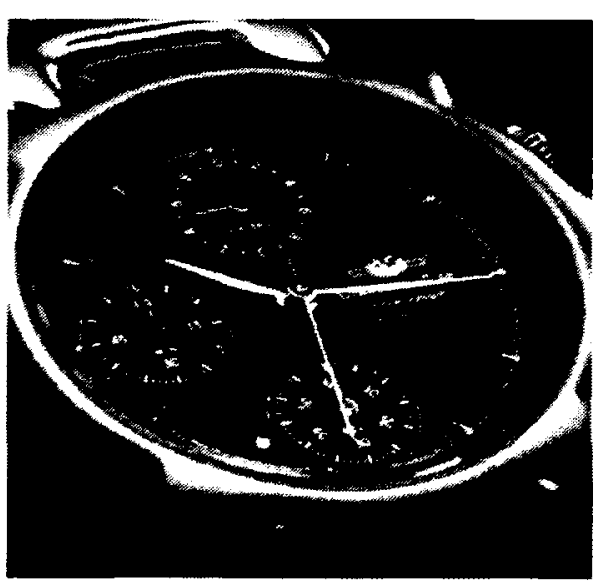

(c) IHAC

Figure 8.3: Figure 8.3(a) is an image halved in the spatial domain using IHS algorithm where the following resultant images from halving algorithms are compared with: Figure 8.3(b) integer based IIHAC and Figure 8.3(c) floating-point based IHAC. The PSNR values are 40.63 and 43.01 respectively. 


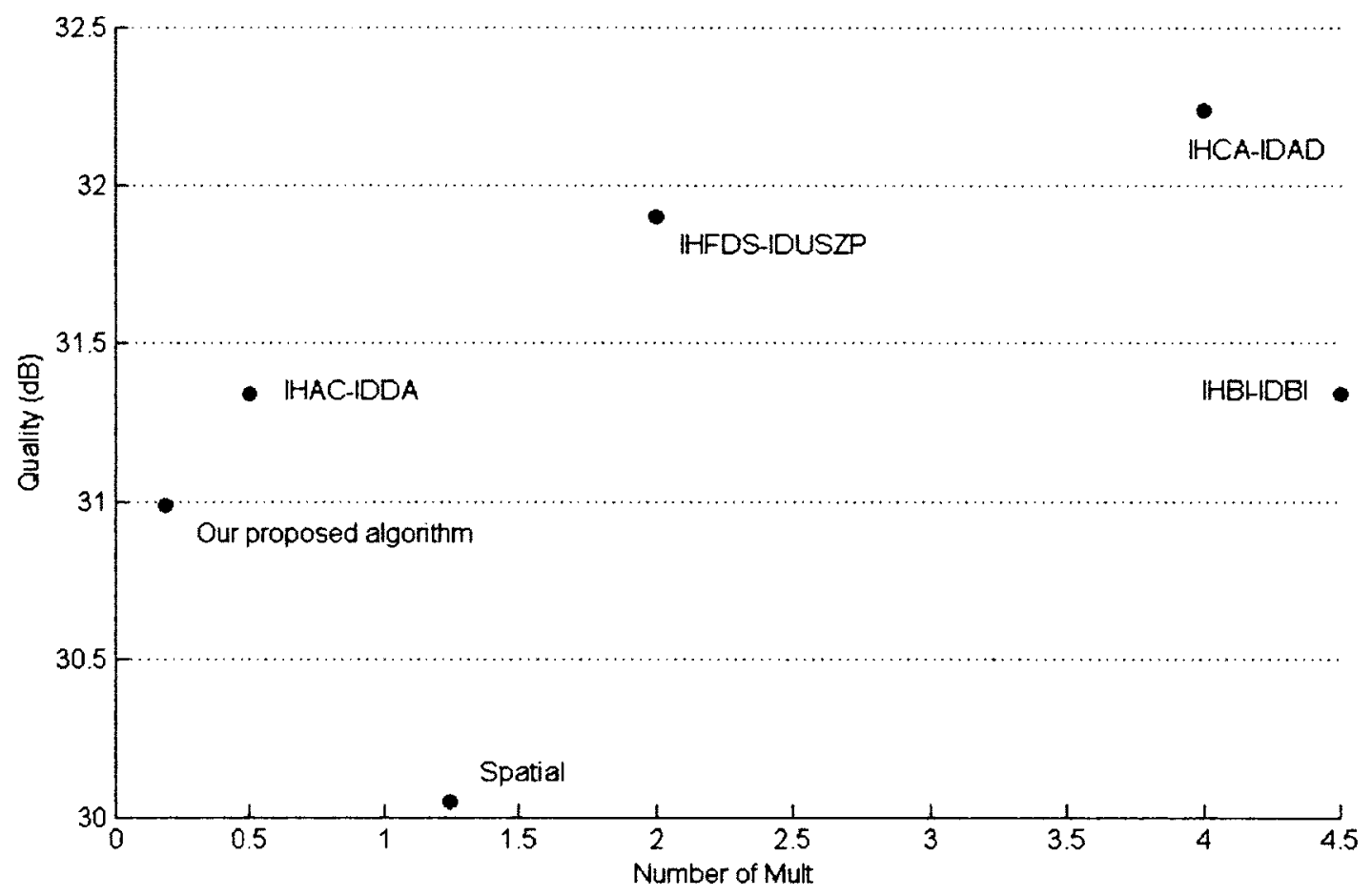

Figure 8.4: Average image quality versus number of multiplications of various resizing algorithms.

Figure 8.4 shows the average PSNR versus the number of multiplications. For clarity, we have refrained from displaying the number of additions. Figure 8.4 plots the two performance metrics of quality and computation simultaneously in one graph. There appears a correlation, the greater the algorithm's computational cost the greater the image quality. Our proposed efficient algorithms clearly has the lowest cost but provides acceptable image quality compared to other algorithms and is superior to spatial resizing in both quality and cost. So our proposed algorithm provides acceptable trade off between image quality and cost. 


\section{Chapter 9}

\section{Conclusions and Future Work}

\subsection{Introduction}

The main goal of the research presented in this thesis was to propose methods to resize H.264/AVC frames in the compressed domain. Resizing H.264/AVC video for storage or transmission is common problem in many application areas, such as mobile phone industry. Systems commonly resize video in the spatial domain but it is undesirable due to inherent high computational overhead associated with decompression and compression operations. A more attractive approach is to resize the video directly in the compressed format, which will reduce the computational cost of the overall resizing process.

In this thesis, we proposed three contributions to meet our goal of resizing H.264/AVC frames in the compressed domain. The next section will outline the results of these contributions.

\subsection{Concluding Remarks}

This section will summarize the results of the thesis contributions:

a) Designing algorithms to resize H.264/AVC frames in the DCT space. We developed thirteen algorithms to resize H.264/AVC frames in the DCT domain that are based on $8 \times 8$ DCT resize algorithms. Even though the H.264/AVC transform and quantization process departs from previous video coding standards, we were able to adapt these $8 \times 8$ DCT resize algorithms to change the resolution of an H.264/AVC encoded frames. Our experimental results showed the images generated from these modified algorithms had an image quality similar or better than comparable spatial domain techniques. Also, most of the adapted algorithms had lower computational cost than spatial domain resizing. 
b) Quantization step can be removed from the resizing process. In the beginning of our research, we assumed that the inverse quantization had to be applied to the H.264/AVC DCT coefficients before DCT resizing. From experiments, we discovered that we can skip the inverse and forward quantization step in the resizing process; hence, reducing the overall process computational cost. The reason is that the conversion matrix used to compose or decompose DCT blocks are orthonormal. This implies that conversion matrix transforms are energy preserving, that is, the energy in the resized image is the same as the original image. Therefore, applying forward quantization is not required before DCT resizing. Our results showed that resizing process including quantization only had a slight improvement in bitrate savings over one the excluded quantization.

c) Efficient resizing algorithms in DCT domain. Several resize algorithms employ conversion matrix to compose or decompose blocks of DCT coefficients in their implementation. We developed an fixed-point approximation of a conversion matrix that is used in IHAC and IDDA resize algorithms. Matrix multiplications with fixed-point conversion matrix are multiplier-free, which enhanced computational efficiency of our proposed halving and doubling algorithms. Images generated from our fixed-point resize algorithms provided PSNR values negligibly lower than their floating-point implementations but substantial higher quality than images resized using bilinear interpolation for halving and zeroorder interpolation for doubling in the spatial domain. Systems utilizing our proposed integer-based algorithms will produce images with good quality at a greatly reduced computational cost compare to resizing images in the spatial domain.

\subsection{Future Work}

Resizing H.264/AVC frames in the compressed domain has shown promise of providing good image quality with increase computational efficiency. The next step is to implement these algorithms in an H.264/AVC encoder, such as JM reference software ${ }^{1}$.

\footnotetext{
${ }^{1}$ H.264/AVC JM Reference Software, http://iphome.hhi.de/suehring/tml/
} 
Our proposed efficient resizing algorithms can only do image halving and doubling and only on conversion matrix of size $\mathbf{A}_{(2,2)}$. The approach of creating an integer approximation of the conversion matrix can be extended so one can resize images by integral or arbitrary factors. There are several resizing algorithms that use conversion matrix for composition and decomposition such as IHCA, IDAD, LMDS, LMUS and HRA, which would be good candidates for this approach. This would be a logical avenue for future research. 


\section{Appendix A}

\section{PSNR for Image Halving and Doubling Experiments}

This appendix outlines the results of image halving and doubling experiments.

Table A.1: Experiment 1. PSNR values computed by comparing an image halved in the spatial domain using bicubic interpolation with anti-aliasing against images generated from various halving algorithms.

\begin{tabular}{r|c|c|c|c|c}
\hline \hline Image & IHS & IHBI & IHFDS & IHAC & IHCA \\
\hline \hline aerial_buildings.raw & 39.55 & 37.53 & 39.11 & 39.05 & 35.89 \\
apc.raw & 45.61 & 44.6 & 45.81 & 45.72 & 44.25 \\
apc_two_vehs1.raw & 42.2 & 40.81 & 42.19 & 42.1 & 40.16 \\
apc_two_vehs2.raw & 42.07 & 40.68 & 42.01 & 41.93 & 40.03 \\
apc_two_vehs3.raw & 46.04 & 44.47 & 46.02 & 45.89 & 43.87 \\
Barbara.raw & 36.45 & 35.72 & 37.83 & 37.8 & 36.88 \\
boat.raw & 41.8 & 40 & 41.34 & 41.28 & 39.02 \\
cameraman.raw & 46.97 & 42.64 & 44.56 & 44.46 & 40.62 \\
Elaine.raw & 45.42 & 43.1 & 45.4 & 45.31 & 43.24 \\
Goldhill.raw & 42.72 & 41.12 & 42.88 & 42.79 & 40.83 \\
house.raw & 52.18 & 45.94 & 49.43 & 49.25 & 45.73 \\
jetplane.raw & 44.31 & 41.03 & 42.84 & 42.76 & 39.39 \\
lake.raw & 42.83 & 39.95 & 41.97 & 41.89 & 38.63 \\
landed_jet.raw & 47.08 & 45.3 & 46.98 & 46.9 & 44.64 \\
Lena.raw & 45.52 & 42.69 & 44.72 & 44.62 & 41.58 \\
livingroom.raw & 41.25 & 39.67 & 41.26 & 41.2 & 38.71 \\
mandril.raw & 35.82 & 34.9 & 36.17 & 36.13 & 34.54 \\
peppers.raw & 44.9 & 41.76 & 43.96 & 43.87 & 41.13 \\
pirate.raw & 42.7 & 40.83 & 42.58 & 42.49 & 39.96 \\
tank_front.raw & 42.7 & 41.62 & 42.9 & 42.82 & 41.01 \\
tank_side.raw & 43.05 & 41.79 & 43.05 & 42.96 & 41.18 \\
tank_top.raw & 44.58 & 43.39 & 44.72 & 44.62 & 42.85 \\
truck.raw & 44.71 & 43.22 & 44.79 & 44.68 & 42.65 \\
walkbridge.raw & 39.1 & 37.56 & 39.12 & 39.07 & 36.76 \\
watch.raw & 42.6 & 38.79 & 40.57 & 40.51 & 36.53 \\
woman_darkhair.raw & 50.07 & 47 & 49.48 & 49.35 & 47.12 \\
\hline Mean & 43.50 & 41.36 & 43.10 & 43.01 & 40.62 \\
\hline \hline
\end{tabular}


Table A.2: Experiment 2. PSNR values computed by comparing the original images with images that were doubled from compressed halved images using various doubling algorithms. Bicubic interpolation with anti-aliasing was used to halve the original images to create the compressed halved images.

\begin{tabular}{r|c|c|c|c|c}
\hline Image & IDS & IDZOI & IDUSZP & IDDA & IDAD \\
\hline \hline aerial_buildings.raw & 25.5 & 25.04 & 26.6 & 26.6 & 27.27 \\
apc.raw & 33.32 & 33.04 & 33.87 & 33.87 & 34.14 \\
apc_two_vehs1.raw & 29.16 & 28.81 & 29.87 & 29.87 & 30.21 \\
apc_two_vehs2.raw & 28.97 & 28.64 & 29.67 & 29.67 & 30.02 \\
apc_two_vehs3.raw & 33.33 & 32.88 & 34.35 & 34.35 & 34.76 \\
Barbara.raw & 25.3 & 25.15 & 25.48 & 25.48 & 25.39 \\
boat.raw & 28.37 & 27.94 & 29.34 & 29.34 & 29.82 \\
cameraman.raw & 31.02 & 30.2 & 33.47 & 33.47 & 34.99 \\
Elaine.raw & 31.6 & 31.15 & 32.65 & 32.65 & 32.88 \\
Goldhill.raw & 30.13 & 29.74 & 30.97 & 30.97 & 31.33 \\
house.raw & 35.89 & 34.71 & 40.42 & 40.42 & 42.12 \\
jetplane.raw & 28.76 & 28.17 & 30.45 & 30.45 & 31.29 \\
lake.raw & 28.15 & 27.57 & 29.65 & 29.65 & 30.41 \\
landed_jet.raw & 34.14 & 33.66 & 35.31 & 35.31 & 35.74 \\
Lena.raw & 31.39 & 30.76 & 33.03 & 33.03 & 33.79 \\
livingroom.raw & 28.09 & 27.71 & 28.9 & 28.9 & 29.44 \\
mandril.raw & 23.12 & 22.92 & 23.48 & 23.48 & 23.71 \\
peppers.raw & 29.86 & 29.3 & 31.44 & 31.44 & 31.98 \\
pirate.raw & 29.34 & 28.89 & 30.37 & 30.37 & 30.87 \\
tankfront.raw & 30.07 & 29.78 & 30.64 & 30.64 & 30.97 \\
tank_side.raw & 30.2 & 29.88 & 30.83 & 30.83 & 31.13 \\
tank_top.raw & 31.9 & 31.61 & 32.49 & 32.49 & 32.81 \\
truck.raw & 32.01 & 31.61 & 32.87 & 32.87 & 33.27 \\
walkbridge.raw & 25.94 & 25.58 & 26.67 & 26.67 & 27.10 \\
watch.raw & 25.9 & 25.32 & 27.47 & 27.47 & 28.37 \\
woman_darkhair.raw & 37.09 & 36.23 & 39.64 & 39.64 & 40.31 \\
\hline \hline Mean & 29.89 & 29.42 & 31.08 & 31.08 & 31.62 \\
\hline & & & & & \\
\hline
\end{tabular}


Table A.3: Experiment 3. PSNR values computed by comparing the original images with images generated from various halving and doubling algorithms.

\begin{tabular}{r|c|c|c|c|c}
\hline \hline Image & IHS & IHBI & IHFDS & IHAC & IHCA \\
& IDS & IDZOI & IDUSZP & IDDA & IDAD \\
\hline aerial_buildings.raw & 25.66 & 25.28 & 26.85 & 26.85 & 27.9 \\
apc.raw & 33.52 & 33.31 & 34.12 & 34.12 & 34.54 \\
apc_two_vehs1.raw & 29.35 & 29.08 & 30.11 & 30.11 & 30.65 \\
apc_two_vehs.raw & 29.17 & 28.91 & 29.92 & 29.92 & 30.46 \\
apc_two_vehs3.raw & 33.51 & 33.16 & 34.61 & 34.61 & 35.28 \\
Barbara.raw & 25.63 & 25.54 & 25.73 & 25.73 & 25.7 \\
boat.raw & 28.55 & 28.2 & 29.61 & 29.61 & 30.36 \\
cameraman.raw & 31.09 & 30.43 & 33.77 & 33.77 & 36.37 \\
Elaine.raw & 31.74 & 31.41 & 32.86 & 32.86 & 33.27 \\
Goldhill.raw & 30.35 & 30.04 & 31.24 & 31.24 & 31.83 \\
house.raw & 35.91 & 35.01 & 40.84 & 40.84 & 44.26 \\
jetplane.raw & 28.86 & 28.39 & 30.69 & 30.69 & 31.99 \\
lake.raw & 28.28 & 27.82 & 29.89 & 29.89 & 31.10 \\
landed_jet.raw & 34.28 & 33.92 & 35.55 & 35.55 & 36.26 \\
Lena.raw & 31.52 & 31.02 & 33.3 & 33.3 & 34.53 \\
livingroom.raw & 28.28 & 27.98 & 29.15 & 29.15 & 29.98 \\
mandril.raw & 23.35 & 23.2 & 23.72 & 23.72 & 24.08 \\
peppers.raw & 29.96 & 29.53 & 31.67 & 31.67 & 32.54 \\
pirate.raw & 29.52 & 29.16 & 30.62 & 30.62 & 31.43 \\
tank_front.raw & 30.28 & 30.05 & 30.89 & 30.89 & 31.40 \\
tank_side.raw & 30.4 & 30.15 & 31.08 & 31.08 & 31.57 \\
tank_top.raw & 32.09 & 31.87 & 32.72 & 32.72 & 33.22 \\
truck.raw & 32.2 & 31.89 & 33.13 & 33.13 & 33.76 \\
wamalkbridge.raw & 26.14 & 25.86 & 26.92 & 26.92 & 27.59 \\
watch.raw & 25.98 & 25.51 & 27.69 & 27.69 & 29.14 \\
woman_darkhair.raw & 37.15 & 36.53 & 39.94 & 39.94 & 41.10 \\
\hline Mean & 30.05 & 29.69 & 31.34 & 31.34 & 32.24 \\
\hline \hline
\end{tabular}




\section{Appendix B}

\section{PSNR for Image Resizing by Integral Factors}

This appendix contains the experimental results from algorithms that resize images by integral factors.

Table B.1: Experiment 4. PSNR values for LMDS and LMUS algorithms.

\begin{tabular}{|c|c|c|c|c|c|c|}
\hline Images & $\mathrm{L}$ & M & $\begin{array}{c}\text { DS-PSNR (dB) } \\
\text { LMDS }\end{array}$ & $\begin{array}{l}\text { US-PSNR (dB) } \\
\text { LMUS }\end{array}$ & $\begin{array}{l}\text { DS-US-PSNR } \\
\text { LMDS/LMUS }\end{array}$ & $\begin{array}{c}\text { DS-US-PSNR } \\
\text { bilinear/bilinear }\end{array}$ \\
\hline \multirow[t]{10}{*}{ Boat } & $\overline{1}$ & 2 & 36.93 & 30.03 & 31.01 & 29.18 \\
\hline & 2 & 2 & 34.90 & 29.06 & 30.36 & 27.98 \\
\hline & 2 & 3 & 30.89 & 26.58 & 27.95 & 26.54 \\
\hline & 2 & 5 & 30.73 & 21.20 & 25.61 & 24.83 \\
\hline & 3 & 2 & 30.53 & 27.33 & 28.86 & 27.05 \\
\hline & 3 & 3 & 28.67 & 25.60 & 27.10 & 25.92 \\
\hline & 3 & 4 & 30.75 & 25.18 & 25.98 & 25.04 \\
\hline & 3 & 5 & 28.95 & 23.68 & 25.16 & 24.46 \\
\hline & 4 & 4 & 34.10 & 25.06 & 25.64 & 24.55 \\
\hline & 5 & 5 & 28.46 & 22.29 & 24.28 & 23.72 \\
\hline \multirow[t]{10}{*}{ Goldhill } & 1 & 2 & 39.83 & 33.13 & 34.14 & 32.31 \\
\hline & 2 & 2 & 37.06 & 30.70 & 31.83 & 29.79 \\
\hline & 2 & 3 & 33.57 & 29.06 & 30.24 & 28.84 \\
\hline & 2 & 5 & 32.77 & 23.27 & 28.19 & 27.21 \\
\hline & 3 & 2 & 33.17 & 28.91 & 30.06 & 28.76 \\
\hline & 3 & 3 & 31.43 & 27.84 & 29.03 & 28.05 \\
\hline & 3 & 4 & 33.11 & 27.48 & 28.29 & 27.27 \\
\hline & 3 & 5 & 31.27 & 25.97 & 27.51 & 26.73 \\
\hline & 4 & 4 & 36.36 & 27.18 & 27.73 & 26.71 \\
\hline & 5 & 5 & 30.99 & 24.56 & 26.43 & 25.88 \\
\hline
\end{tabular}




\begin{tabular}{|c|c|c|c|c|c|c|}
\hline Images & $\mathrm{L}$ & M & $\begin{array}{c}\text { DS-PSNR (dB) } \\
\text { LMDS }\end{array}$ & $\begin{array}{l}\text { US-PSNR (dB) } \\
\text { LMUS }\end{array}$ & $\begin{array}{l}\text { DS-US-PSNR } \\
\text { LMDS/LMUS }\end{array}$ & $\begin{array}{c}\text { DS-US-PSNR } \\
\text { bilinear/bilinear }\end{array}$ \\
\hline \multirow[t]{10}{*}{ Lena } & 1 & 2 & 39.18 & 34.15 & 35.73 & 33.09 \\
\hline & 2 & 2 & 37.22 & 32.68 & 34.53 & 31.40 \\
\hline & 2 & 3 & 31.43 & 29.43 & 31.10 & 29.60 \\
\hline & 2 & 5 & 30.59 & 20.76 & 27.80 & 27.03 \\
\hline & 3 & 2 & 32.45 & 30.65 & 32.44 & 30.38 \\
\hline & 3 & 3 & 29.56 & 28.42 & 30.19 & 29.00 \\
\hline & 3 & 4 & 32.26 & 28.13 & 29.14 & 27.73 \\
\hline & 3 & 5 & 29.52 & 25.05 & 27.46 & 26.78 \\
\hline & 4 & 4 & 35.17 & 28.04 & 28.96 & 27.30 \\
\hline & 5 & 5 & 29.05 & 23.74 & 26.74 & 26.18 \\
\hline \multirow[t]{10}{*}{ Watch } & 1 & 2 & 35.06 & 31.13 & 32.86 & 29.73 \\
\hline & 2 & 2 & 32.08 & 27.28 & 29.14 & 25.87 \\
\hline & 2 & 3 & 28.88 & 25.17 & 27.00 & 24.74 \\
\hline & 2 & 5 & 27.62 & 18.12 & 24.37 & 22.90 \\
\hline & 3 & 2 & 29.20 & 24.50 & 26.35 & 24.23 \\
\hline & 3 & 3 & 27.57 & 23.48 & 25.24 & 23.51 \\
\hline & 3 & 4 & 29.16 & 23.04 & 24.31 & 22.74 \\
\hline & 3 & 5 & 26.61 & 21.48 & 23.52 & 22.19 \\
\hline & 4 & 4 & 30.46 & 22.60 & 23.47 & 21.87 \\
\hline & 5 & 5 & 25.70 & 20.25 & 22.09 & 20.80 \\
\hline
\end{tabular}




\section{Appendix C}

\section{PSNR for Arbitrary Resizing Algorithms}

This appendix outlines experimental results of arbitrary resizing algorithms.

Table C.1: Experiment 5. PSNR values after arbitrary resizing using various scaling factors and algorithms.

\begin{tabular}{r|c|c|c}
\hline \multicolumn{4}{c}{$\frac{2}{3} \times \frac{4}{5}$} \\
\hline \hline Image & HRA & DURA & UDRA \\
\hline Boat & 31.92 & 26.86 & 32.41 \\
Goldhill & 34.31 & 29.62 & 34.92 \\
Lena & 34.31 & 28.56 & 34.79 \\
Watch & 30.00 & 25.29 & 30.27 \\
\hline \hline \multicolumn{4}{c}{$\frac{3}{2} \times \frac{5}{4}$} \\
\hline \hline Image & HRA & DURA & UDRA \\
\hline Boat & 36.86 & 28.15 & 38.30 \\
Goldhill & 38.38 & 31.07 & 39.43 \\
Lena & 40.15 & 31.03 & 40.86 \\
Watch & 35.86 & 27.02 & 36.47 \\
\hline \multicolumn{4}{|c}{$\frac{2}{3} \times \frac{3}{2}$} \\
\hline \hline Image & HRA & DURA & UDRA \\
\hline Boat & 34.54 & 31.30 & 34.88 \\
Goldhill & 35.96 & 33.14 & 36.33 \\
Lena & 37.62 & 34.69 & 37.98 \\
Watch & 31.92 & 28.95 & 32.13 \\
\hline \multicolumn{4}{c}{$\frac{3}{2} \times \frac{2}{3}$} \\
\hline \hline Image & HRA & DURA & UDRA \\
\hline Boat & 33.75 & 31.27 & 33.99 \\
Goldhill & 36.53 & 33.63 & 36.92 \\
Lena & 36.70 & 33.48 & 37.04 \\
Watch & 33.01 & 29.35 & 33.17 \\
\hline \hline
\end{tabular}


Table C.2: Experiment 6. PSNR values after arbitrary resizing followed by inverse resizing.

\begin{tabular}{r|c|c|c}
\hline \multicolumn{4}{|c}{$\frac{2}{3} \times \frac{4}{5}$} \\
\hline \hline Image & HRA/HRA & DURA/DURA & UDRA/UDRA \\
\hline Boat & 34.96 & 25.16 & 33.88 \\
Goldhill & 35.81 & 27.51 & 34.90 \\
Lena & 38.34 & 27.46 & 36.99 \\
Watch & 34.38 & 23.52 & 33.10 \\
\hline \multicolumn{4}{|c}{$\frac{3}{2} \times \frac{5}{4}$} \\
\hline \hline Image & HRA/HRA & DURA/DURA & UDRA/UDRA \\
\hline Boat & 55.19 & 26.57 & 44.20 \\
Goldhill & 55.17 & 29.19 & 46.73 \\
Lena & 55.20 & 29.72 & 48.60 \\
Watch & 56.31 & 25.50 & 45.78 \\
\hline \hline \multicolumn{4}{|c}{$\frac{2}{3} \times \frac{3}{2}$} \\
\hline Image & HRA/HRA & DURA/DURA & UDRA/UDRA \\
\hline Boat & 39.09 & 28.86 & 37.88 \\
Goldhill & 37.60 & 30.06 & 36.87 \\
Lena & 40.40 & 32.44 & 39.3 \\
Watch & 35.47 & 26.35 & 34.33 \\
\hline \hline
\end{tabular}




\section{Appendix D}

\section{Image Sample Set}

All the images are 8-bit grey scale with resolution size of $512 \times 512$ pixels. The images were downloaded from the University of Southern California, Signal and Image Processing Institute (USC-SIPI) Image Database (http://sipi.usc.edu/database/).

Table D.1: Images that are members of the sample set.

\begin{tabular}{|c|c|c|}
\hline Ser. & File Name & Comments \\
\hline$\overline{1} 1$ & aerial_buildings.raw & An aerial photograph of several large buildings. \\
\hline 2 & apc.raw & A side view of an armoured personnel carrier (APC). \\
\hline 3 & apc_two_vehs1.raw & A picture of van, truck and APC. \\
\hline 4 & apc_two_vehs2.raw & An another picture of van, truck and APC. \\
\hline 5 & apc_two_vehs3.raw & A picture of van, station wagon and APC. \\
\hline 6 & Barbara.raw & A picture of a woman sitting on the floor. \\
\hline 7 & boat.raw & A picture of several moored fishing boats. \\
\hline 8 & cameraman.raw & A picture of a cameraman. \\
\hline 9 & Elaine.raw & A picture of a woman wearing a hat. \\
\hline 10 & Goldhill.raw & A street scene of an old English village. \\
\hline 11 & house.raw & A picture of a house. \\
\hline 12 & jetplane.raw & A picture of a flying F-16 fighter jet. \\
\hline 13 & lake.raw & A landscape scene of a lake. \\
\hline 14 & landed_jet.raw & A aerial image of landed fighter jet in the desert. \\
\hline 15 & Lena.raw & The famous image of the Playboy centrefold, Lena. \\
\hline 16 & livingroom.raw & A picture displaying a man and woman in a living room. \\
\hline 17 & mandril.raw & A picture of a baboon face. \\
\hline 18 & peppers.raw & A picture of various peppers. \\
\hline 19 & pirate.raw & A picture of an actor dressed as a pirate. \\
\hline 20 & tank_front.raw & An image of a front of a tank. \\
\hline 21 & tank_side.raw & An image of a side of a tank. \\
\hline 22 & tank_top.raw & An image of a tank from the top. \\
\hline 23 & truck.raw & An image of an military truck in the desert. \\
\hline 24 & walkbridge.raw & A picture of a walking bridge. \\
\hline 25 & watch.raw & A close up picture of a wrist watch. \\
\hline 26 & woman_blonde.raw & A closeup image of a blonde haired woman. \\
\hline 27 & woman_darkhair.raw & A closeup image of a dark haired woman. \\
\hline
\end{tabular}




\section{Appendix E}

\section{Hybrid Resizing Algorithm (HRA) Source Code}

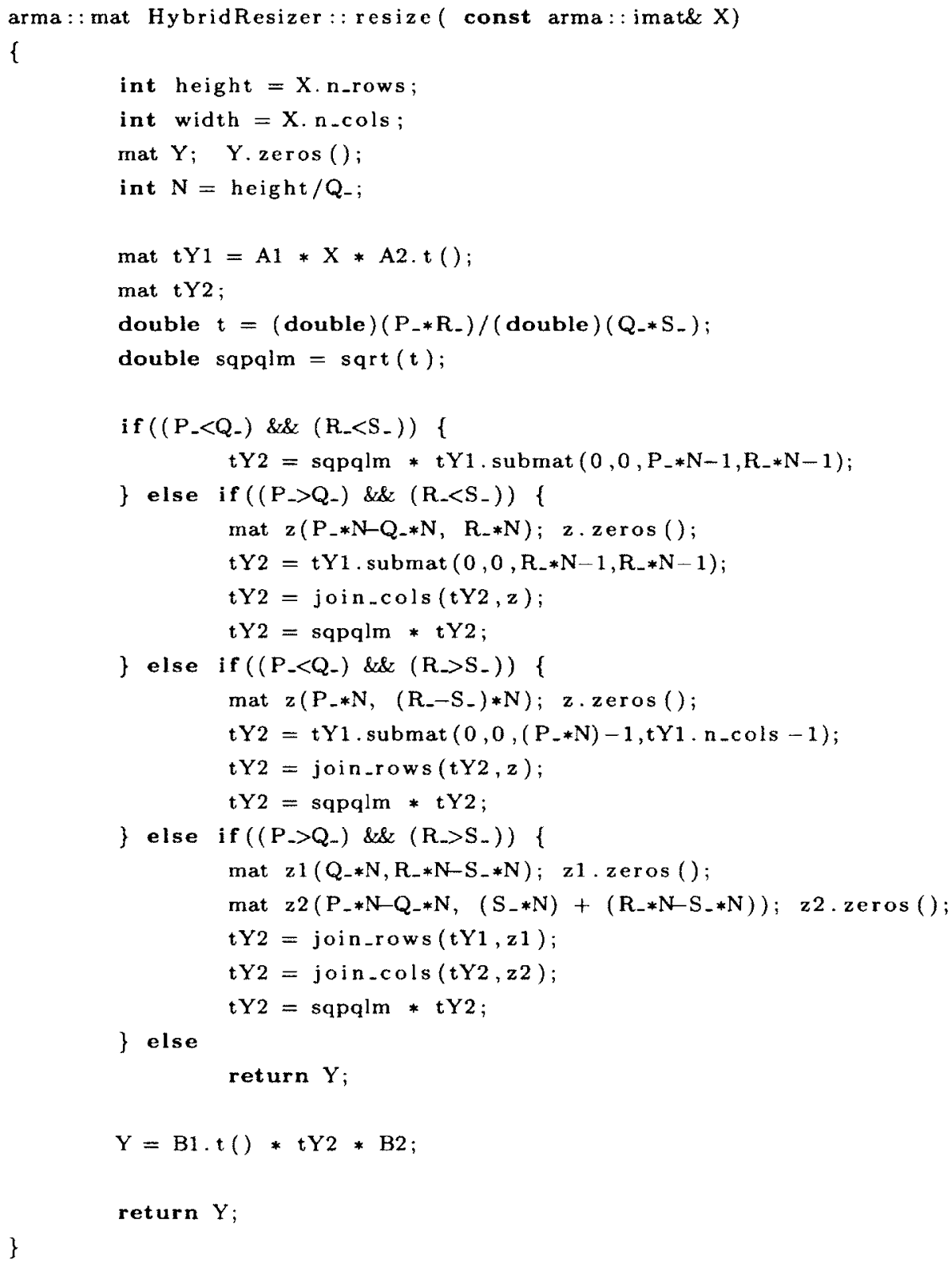




\section{Bibliography}

[1] Advanced video coding for generic audiovisual services, International Telecommunication Union Std. H.264, Rev. 16, January 2012. [Online]. Available: http://www.itu.int/rec/T-REC-H.264

[2] Information technology - Coding of audio-visual objects - Part 10: Advanced Video Coding, ISO Std. ISO/IEC 14496-10:2012, January 2012. [Online]. Available: http://www.iso.org/iso/home/store/catalogue_ics/catalogue_detail_ ics.htm?csnumber $=61490$

[3] H. S. Malvar, A. Hallapuro, M. Karczewicz, and L. Kerofsky, "Low-complexity transform and quantization in H.264/AVC," IEEE Transaction Circuits and Systems Video Technology, vol. 13, pp. 598-603, July 2003.

[4] A. Luthra, G. Sullivan, and T. Wiegand, "Introduction to the special issue on the H.264/AVC video coding standard," IEEE Transaction on Circuits and Systems for Video Technology, vol. 13, no. 7, July 2003.

[5] C. Salazar and T. D. Tran, "On resizing images in the DCT domain," in IEEE International Conference on Image Processing, vol. 4, October 2004, pp. 27972800 .

[6] _ - "A more efficient and video friendly spatial resizing algorithm," in IEEE International Conference on Image Processing, vol. 4, October 2006, pp. 877-880.

[7] S.-F. Chang and D. G. Messerchmitt, "Manipulation and compositing of MCDCT compressed video," IEEE Journal on Selected Areas in Communications, vol. 13, no. 1, pp. 1-11, January 1995.

[8] N. Merhav and V. Bhaskaran, "Fast inverse motion compensation algorithms for MPEG-2 and for partial DCT information," 1996.

[9] Y.-R. Lee and C.-W. Lin, "DCT-domain spatial transcoding using generalized DCT decimation," in IEEE International Conference on Image Processing, vol. 1, September 2005, pp. 821-824.

[10] _- "Visual quality enhancement in dct-domain spatial downscaling transcoding using generalized dct decimation," Circuits and Systems for Video Technology, IEEE Transactions on, vol. 17, no. 8, pp. 1079-1084, 2007.

[11] N. Merhav and V. Bhaskaran, "Fast algorithms for DCT-domain image downsampling and for inverse motion compensation," IEEE Transaction on Circuits and Systems for Video Technology, vol. 7, no. 3, pp. 486-476, June 1997. 
[12] R. Dugad and N. Ahuja, "A fast scheme for image size change in the compressed domain," IEEE Transaction on Circuits and Systems for Video Technology, vol. 11, no. 4, pp. 461-474, April 2001

[13] J. Mukherjee and S. K. Mitra, "Arbitrary resizing of images in the DCT space," in IEE Proceeding Vision, Image and Signal Processing, vol. 152, 2005, pp. 155164.

[14] J. Mukhopadhyay, Image and Video Processing in the Compressed Domain. A Chapman and Hall Book, 2011.

[15] I. Richardson, The H.264 Advanced Video Compression Standard, 2nd ed. A John Wiley and Sons Ltd., 2010.

[16] R. D. Dony, The Transform and Data Compression Handbook. CRC Press, 2000, ch. 1. Karhunen-Loeve Transform.

[17] K. Sayood, Introduction to Data Compression, 3rd ed. Elsevier Inc., 2006.

[18] N. Ahmed, T. Natarajan, and K. R. Rao, "Discrete cosine transform," IEEE Transaction of Computers, pp. 90-93, January 1974.

[19] A. Hallapuro, M. Karczewicz, and H. S. Malvar, "Low-complexity transform and quantization," in Joint Video Team (JVT) of ISO/IEC MPEG and ITU-VCEG, January 2002.

[20] S.-H. Jung, S. K. Mitra, and J. Mukherjee, "Subband DCT: Definition, analysis, and applications," IEEE Transaction on Circuits and Systems for Video Technology, vol. 6, no. 3, pp. 273-286, June 1996.

[21] J. Mukherjee and S. K. Mitra, "Image resizing in the compressed domain using subband DCT," IEEE Transaction on Circuits and Systems for Video Technology, vol. 12, no. 7, pp. 620-627, July 2002.

[22] J. Jiang and G. Feng, "The spatial relationship of DCT coefficients between a block and its sub-blocks," IEEE Transaction on Signal Processing, vol. 50, no. 5, pp. 1160-1169, May 2002.

[23] T. D. Tran and C. Salazar, "A complexity scalable universal DCT domain image resizing algorithm," IEEE Transaction on Circuits and Systems for Video Technology, vol. 17, no. 4, pp. 495-499, April 2007.

[24] H.264 Bandwidth/Latency/Quality Tradeoffs, Motion Imagery Standards Board Std. EG 0904, September 2009. [Online]. Available: http://www.gwg.nga.mil/ misb/docs/eg/EG0904.pdf

[25] V. K. Goyal, "Theoretical foundations of transform coding," IEEE Signal Processing Magazine, pp. 9-21, September 2001. 
[26] R. Gray and D. Neuhoff, "Quantization," Information Theory, IEEE Transactions on, vol. 44, pp. 2325-2383, 1998.

[27] J. Hefferon, Linear Algebra: (Textbook). Orange Grove Books, 2009. [Online]. Available: http://joshua.smcvt.edu/linearalgebra/

[28] Y.-R. Lee, C.-W. Lin, and C.-C. Kao, "A dct-domain video transcoder for spatial resolution downconversion," in Proceedings of the 5th International Conference on Recent Advances in Visual Information Systems, ser. VISUAL '02. London, UK, UK: Springer-Verlag, 2002, pp. 207-218. [Online]. Available: http://dl.acm.org/citation.cfm?id=647062.714601

[29] S. A. Martucci, "Image resizing in the discrete cosine transform domain," in IEEE International Conference on Image Processing, vol. 2, October 1995, pp. $244-247$.

[30] J. Mukhopadhyay and S. Mitra, "Resizing of images in the DCT space by arbitrary factors," in Image Processing, 2004. ICIP '04. 2004 Intermational Conference on, vol. 4, October 2004, pp. 2801-2804.

[31] Y. Noguchi, D. G. Messerschmitt, and S.-F. Chang, "MPEG video compositing in the compressed domain," IEEE, pp. 596-599, 1996.

[32] Y. S. Park and H. W. Park, "Arbitrary-ratio image resizing using fast DCT of composite length for DCT-based transcoder," IEEE Transaction on Image Processing, vol. 15, pp. 494-500, February 2006.

[33] H. W. Park, Y. S. Park, and S.-K. Oh, "L/M-fold image resizing in block-DCT domain using symmetric convolution," IEEE Transaction on Image Processing, vol. 12 , pp. 1016-1034, September 2003.

[34] V. Patil, R. Kumar, and J. Mukherjee, "A fast arbitrary factor video resizing algorithm," IEEE Transaction on Circuits and Systems for Video Technology, vol. 16, pp. 1164-1171, September 2006.

[35] V. Patil and R. Kumar, "A fast arbitrary factor H.264/AVC video re-sizing algorithm," in IEEE International Conference on Image Processing, vol. 4, October 2007 , pp. $65-68$.

[36] H. Shen, X. Sun, F. Wu, H. Li, and S. Li, "A fast downsizing video transcoder for H.264/AVC with rate-distortion optimal mode decision," in IEEE International Conference on Multimedia and Expo, July 2006, pp. 2017-2020.

[37] I. Shin and H. W. Park, "Adaptive up-sampling method using det for spatial scalability of scalable video coding," IEEE Transaction on Circuits and Systems for Video Technology, vol. 19, pp. 206-213, February 2009. 
[38] H. Shu and L.-P. Chau, "An efficient arbitrary downsizing algorithm for video transcoding," IEEE Transaction on Circuits and Systems for Video Technology, vol. 14, pp. 887-891, June 2004.

[39] N. Szanto and O. Green, "Image resizing in the DCT domain," June 2008. [Online]. Available: https://sipl.technion.ac.il/Info/Teaching_Projects_ Image-Resize_e.shtml

[40] H. Shu and L.-P. Chau, "The realization of arbitrary downsizing video transcoding," IEEE Transaction on Circuits and Systems for Video Technology, vol. 16, pp. 540 546, April 2006.

[41] E.-L. Tan, W.-S. Gan, and S. K. Mitra, "Fast arbitrary resizing of images in the discrete cosine transform domain," Image Processing, IET, vol. 5, pp. 73-86, February 2011.

[42] I. Richardson, Transform and Quantization in H.264/AVC. VCodex Ltd White Paper, April 2009. [Online]. Available: http://http://www.vcodex.com/images/ uploaded/340655053133585.pdf/

[43] S. Wenger, M. Hannuksela, T. Stockhammer, M. Westerlund, and D. Singer, "RTP payload format for H.264 video," RFC 3984, February 2005. [Online]. Available: www.ietf.org/rfc/rfc3984.txt

[44] Z. Wang, H. R. Sheikh, and A. C. Bovik, "No-reference perceptual quality assessment of JPEG compressed images," in IEEE International Conference on Image Processing, vol. 1, Rochester, NY, USA, September 2002, pp. 477-480.

[45] Z. Wang, A. Bovik, H. Sheikh, and E. Simoncelli, "Image quality assessment: from error visibility to structural similarity," Image Processing, IEEE Transactions on, vol. 13, pp. 600-612, April 2004.

[46] Z. Wang and A. Bovik, "Mean squared error: Love it or leave it? a new look at signal fidelity measures," Signal Processing Magazine, IEEE, vol. 26, pp. 98-117, January 2009.

[47] T. Wiegand and H. Schwarz, "Source coding: Part I of fundamentals of source and video coding," Foundations and Trends in Signal Processing, vol. 4, pp. 1-222, January 2011. [Online]. Available: http://www.stanford.edu/class/ ee398a/BookWiegandSchwarz.pdf 